Pacific

Journal of

Mathematics

EXCEPTIONAL SURGERY CURVES IN TRIANGULATED 3-MANIFOLDS

MARC LACKENBY 


\title{
EXCEPTIONAL SURGERY CURVES IN TRIANGULATED 3-MANIFOLDS
}

\author{
MARC LACKENBY
}

\begin{abstract}
For the purposes of this paper, Dehn surgery along a curve $K$ in a 3-manifold $M$ with slope $\sigma$ is 'exceptional' if the resulting 3-manifold $M_{K}(\sigma)$ is reducible or a solid torus, or the core of the surgery solid torus has finite order in $\pi_{1}\left(M_{K}(\sigma)\right)$. We show that, providing the exterior of $K$ is irreducible and atoroidal, and the distance between $\sigma$ and the meridian slope is more than one, and a homology condition is satisfied, then there is (up to ambient isotopy) only a finite number of such exceptional surgery curves in a given compact orientable 3manifold $M$, with $\partial M$ a (possibly empty) union of tori. Moreover, there is a simple algorithm to find all these surgery curves, which involves inserting tangles into the 3-simplices of any given triangulation of $M$. As a consequence, we deduce some results about the finiteness of certain unknotting operations on knots in the 3-sphere.
\end{abstract}

\section{Introduction.}

Consider the following motivating problem from knot theory. Let $L$ be a nontrivial knot in $S^{3}$. If $K$ is an unknotted curve disjoint from $L$, then Dehn surgery along $K$ with slope $1 / q$ has the effect of adding $|q|$ full twists to $L$, yielding a knot $L^{\prime}$, say. (See Figure 1.2.) Suppose that $L^{\prime}$ is the unknot, or (more generally) that $L^{\prime}$ has smaller genus than that of $L$. Then, for a given knot $L$, are there only a finitely many possibilities for $q$ and $K$ (up to ambient isotopy keeping $L$ fixed)? The following theorem deals with this question.

Theorem 1.1. Let $L$ be a knot in $S^{3}$ which is not a nontrivial satellite knot. Let $K$ be an unknotted curve in $S^{3}$, disjoint from $L$ and having zero linking number with $L$. Let $q$ be an integer with $|q|>1$. Suppose that $1 / q$ surgery about $K$ yields a knot $L^{\prime}$ with genus $\left(L^{\prime}\right)<\operatorname{genus}(L)$. Then, for a given knot $L$, there are only finitely many possibilities for $K$ and $q$ up to ambient isotopy keeping $L$ fixed, and there is an algorithm to find them all.

Such 'unknotting operations' have been the object of considerable study. For example, the author in [8] dealt with the case where $K$ bounds a disc intersecting $L$ in two points of opposite sign, and proved that if such a 


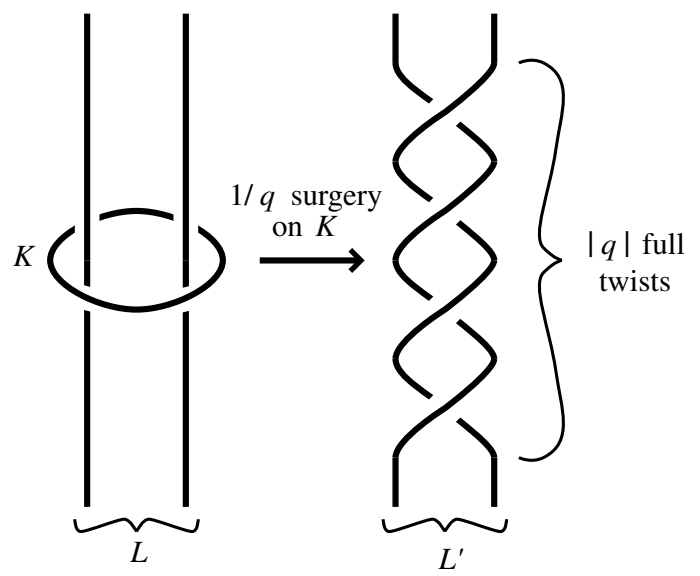

Figure 1.2.

surgery reduces the genus of $L$, then there exists an upper bound on $|q|$ which depends only on $L$, not $K$. Theorem 1.1 gives a great deal more than numerical restrictions on $|q|$. It provides a classification of all such unknotting operations for a given knot $L$, when $|q|>1$ and the linking number of $K$ and $L$ is zero.

Theorem 1.1 is an almost immediate corollary of new results on Dehn surgery. Let $M$ be an arbitrary compact orientable 3-manifold $M$, with $\partial M$ a (possibly empty) union of tori. (In Theorem 1.1, $M$ is the exterior of the knot L.) Our aim is to find the knots $K$ in $M$ with 'exceptional' or 'norm-exceptional' surgeries, which we define as follows.

Definition 1.3. Let $\sigma$ be a slope on $\partial \mathcal{N}(K)$ other than the meridional slope $\mu$. Let $M_{K}(\sigma)$ be the manifold obtained by Dehn surgery along $K$ via the slope $\sigma$. Then $\sigma$ is an exceptional slope and $K$ is an exceptional surgery curve if any of the following holds:

(i) $M_{K}(\sigma)$ is reducible,

(ii) $M_{K}(\sigma)$ is a solid torus, or

(iii) the core of the surgery solid torus has finite order in $\pi_{1}\left(M_{K}(\sigma)\right)$.

Also, $\sigma$ and $K$ are norm-exceptional if there is some $z \in H_{2}(M-\operatorname{int}(\mathcal{N}(K))$, $\partial M)$ which maps to an element $z_{\sigma} \in H_{2}\left(M_{K}(\sigma), \partial M_{K}(\sigma)\right)$, such that the Thurston norm of $z_{\sigma}$ less than the Thurston norm of $z$. (See Section 3 for a definition of the Thurston norm.)

If $K$ satisfies the conditions of Theorem 1.1, then it is a norm-exceptional surgery curve in $M=S^{3}-\operatorname{int}(\mathcal{N}(L))$. The reason for distinguishing normexceptional surgery curves from the exceptional case is that, in the former situation, our results will be slightly weaker. We restrict attention to knots $K$ with irreducible atoroidal exteriors. For technical reasons, we also have to 
assume that $H_{2}(M-\operatorname{int}(\mathcal{N}(K)), \partial M)$ is nontrivial. This implies in particular that the first Betti number of $M$ must be nonzero.

We will show that the problem of finding exceptional surgery curves in a given 3-manifold $M$ falls naturally into two cases, which depend on $\Delta(\sigma, \mu)$, where $\Delta(\sigma, \mu)$ is the intersection number on $\partial \mathcal{N}(K)$ between the surgery slope $\sigma$ and the meridian slope $\mu$. It is not hard to find examples of 3 manifolds $M$ as above containing an infinite number of pairwise non-isotopic surgery curves $K$ with exceptional or norm-exceptional surgery slopes $\sigma$ satisfying $\Delta(\sigma, \mu)=1$. (We will do this in Section 12.) However, the main theorem of this paper asserts that, if $\Delta(\sigma, \mu)>1$, then there is only a finite number of possibilities for $K$ and $\sigma$.

Theorem 1.4. Let $M$ be a compact connected orientable 3-manifold, with $\partial M$ a (possibly empty) union of tori. Let $K$ be a knot in $M$ such that $M-$ $\operatorname{int}(\mathcal{N}(K))$ is irreducible and atoroidal, and with $H_{2}(M-\operatorname{int}(\mathcal{N}(K)), \partial M) \neq$ 0 . Let $\sigma$ be an exceptional or norm-exceptional slope on $\partial \mathcal{N}(K)$, such that $\Delta(\sigma, \mu)>1$, where $\mu$ is the meridian slope on $\partial \mathcal{N}(K)$. Then, for a given $M$, there are at most finitely many possibilities for $K$ and $\sigma$ up to ambient isotopy, and there is an algorithm to find them all.

The algorithm is surprisingly simple. We describe it in Section 2. The input into the algorithm is any triangulation of $M$, or the following generalisation of a triangulation. Let $P$ be a (possibly empty) collection of components of $\partial M$. Then a generalised triangulation of $M$ is a representation of $M-P$ as a union of 3-simplices, with some or all of their faces identified in pairs and then possibly with some subcomplex removed. For example, an ideal triangulation is the case where $P=\partial M$ and where the subcomplex removed is the 0-cells. We will also refer to the case where $P=\emptyset$ as a genuine triangulation.

There is a yet simpler algorithm which deals with the $\sigma$-cable of $K$, which is defined to be the knot in $M$ lying on $\partial \mathcal{N}(K)$ having slope $\sigma$. Recall that a tangle is a (possibly empty) collection of disjoint arcs properly embedded in a 3-ball. Two tangles are identified if there is an isotopy of the 3-ball which is fixed on the boundary and which takes one tangle to the other.

Theorem 1.5. There is a finite collection of tangles, each lying in a 3simplex and with the following property. Let $M, K$ and $\mu$ be as in Theorem 1.4, and let $\sigma$ be an exceptional slope on $\partial \mathcal{N}(K)$ with $\Delta(\sigma, \mu)>1$. Pick any generalised triangulation of $M$. Then, we may insert a tangle from this finite collection into each 3-simplex, in such a way that the tangles join to form a knot which is ambient isotopic to the $\sigma$-cable of $K$. This finite collection of tangles is constructible and is independent of $M, K$ and $\sigma$.

Since these tangles are defined up to isotopy of the 3-simplex $\Delta^{3}$ which is fixed on $\partial \Delta^{3}$, Theorem 1.5 immediately gives that there are only finitely many possibilities in $M$ for the $\sigma$-cable of $K$. 
Theorem 1.5 is a very surprising result. If $\Delta(\sigma, \mu)$ is large, then one would expect the $\sigma$-cable of $K$ to intersect the triangulation of $M$ in a complicated way. But the above result asserts that one can control this complexity. It is also surprising that the same finite collection of tangles should work for all $M$ and all triangulations. Note that in Theorem 1.5 we did not assume that $K$ and $\sigma$ were norm-exceptional. In this case, we have the following slightly weaker result.

Theorem 1.6. Let $M, K$ and $\mu$ be as in Theorem 1.4, and let $\sigma$ be a normexceptional slope on $\partial \mathcal{N}(K)$ with $\Delta(\sigma, \mu)>1$. If $M$ is closed, pick any genuine triangulation of $M$. In the case where $M$ has nonempty boundary, pick any ideal triangulation of $M$. Then, we may insert into each 3-simplex a tangle from the finite collection of Theorem 1.5, in such a way that the tangles join to form a knot which is ambient isotopic to the $\sigma$-cable of $K$.

It is in fact possible to write down explicitly this list of tangles. We will give an algorithm in Section 11 to do this. We have not actually run this algorithm, since the task is fairly lengthy and is more suited to computer implementation.

\section{The algorithm to find all possibilities for $K$ and $\sigma$.}

In this section, we describe the algorithm for finding, in a given 3-manifold $M$, all surgery curves $K$ with exceptional or norm-exceptional surgery slopes $\sigma$, satisfying the conditions of Theorem 1.4. The first (and most important) step is to construct a finite list of possibilities for $K$ and $\sigma$, some of which may be neither norm-exceptional nor exceptional.

We will in Section 11 construct a finite collection of graphs, each embedded in a 3-simplex $\Delta^{3}$. Each graph $G$ meets $\partial \Delta^{3}$ in a collection of vertices. These vertices have valence one and lie in the interior of the 2-simplices of $\partial \Delta^{3}$. There is also a specified regular neighbourhood $\mathcal{N}(G)$ and a collection of disjoint arcs properly embedded in $\Delta^{3}$, lying in $\partial \mathcal{N}(G)$. Each arc is assigned one of two labels, $\gamma$ or $\tau$. Each graph $G$ (together with $\mathcal{N}(G)$ and the arcs $\gamma$ and $\tau$ ) is defined up to isotopy of $\Delta^{3}$ which is fixed on $\partial \Delta^{3}$.

We will show during the course of the paper that it is possible to ambient isotope $K$ and $\sigma$, and to find a handle structure $\mathcal{H}$ on $\mathcal{N}(K)$ with the following properties. Each tetrahedron $\Delta^{3}$ of the generalised triangulation of $M$ intersects the 0 -handles and 1-handles of $\mathcal{H}$ in $\mathcal{N}(G)$, where $G$ is one of the graphs mentioned above. The 2-handles of $\mathcal{H}$ will be attached to $\mathcal{N}(G)$ along the $\operatorname{arcs} \tau$. There will also be a curve of slope $\sigma$ on $\partial \mathcal{N}(K)$ which intersects $\Delta^{3}$ in the arcs $\gamma$.

The algorithm to find all possibilities for $K$ and $\sigma$ therefore proceeds as follows. We insert one of these graphs into each 3 -simplex of the generalised triangulation of $M$. If $\Delta^{2}$ is any 2-simplex of $M$ adjacent to two 3-simplices, and $G_{1}$ and $G_{2}$ are the graphs inserted into these two 3 -simplices, then we 
insist that $\mathcal{N}\left(G_{1}\right) \cap \Delta^{2}=\mathcal{N}\left(G_{2}\right) \cap \Delta^{2}$, and also that the endpoints in $\Delta^{2}$ of the arcs labelled $\gamma$ (respectively, $\tau$ ) in $G_{1}$ correspond precisely with the endpoints in $\Delta^{2}$ of the arcs labelled $\gamma$ (respectively, $\tau$ ) in $G_{2}$. Thus, the graphs $G$ combine to form a graph (which we also call $G$ ) embedded in $M$. We insist that $G$ is disjoint from $\partial M$. The collections of arcs combine to form a collection of curves $\gamma$ and $\tau$ properly embedded in $M$ and lying in $\partial \mathcal{N}(G)$. We insist that each component of $\gamma$ and $\tau$ is a simple closed curve, and that $\gamma$ is connected. Since there are only finitely many 3 -simplices in the representation of $M$ and there are only finitely many possibilities for $\mathcal{N}(G) \cap \Delta^{3}, \tau \cap \Delta^{3}$ and $\gamma \cap \Delta^{3}$ for each 3-simplex $\Delta^{3}$ in $M$, there are only finitely many possibilities for $\mathcal{N}(G), \tau$ and $\gamma$. The handlebody $\mathcal{N}(G)$ and curves $\tau$ specify a handle structure of a 3-manifold $M^{\prime}$, which is a candidate for $\mathcal{N}(K)$. At this stage, $M^{\prime}$ may be something other than a solid torus.

The algorithm proceeds by calculating $H_{1}\left(M^{\prime}\right)$ and $H_{1}\left(\partial M^{\prime}\right)$, and the map $H_{1}\left(\partial M^{\prime}\right) \rightarrow H_{1}\left(M^{\prime}\right)$ induced by inclusion. If this is not the map $\mathbb{Z} \oplus \mathbb{Z} \rightarrow \mathbb{Z}$ that is projection onto one factor, we stop. If it is, we can algorithmically find generators $\lambda$ and $\mu$ of $H_{1}\left(\partial \mathcal{N}\left(M^{\prime}\right)\right)$ such that $\lambda$ maps to $1 \in H_{1}\left(M^{\prime}\right)$, and $\mu$ maps to $0 \in H_{1}\left(M^{\prime}\right)$. We can construct a simple closed curve representative of $\lambda$ on $\partial \mathcal{N}\left(M^{\prime}\right)$ which avoids the 2-handles of $M^{\prime}$. If $M^{\prime}$ is $\mathcal{N}(K)$, then this curve is ambient isotopic in $M$ to $K$. The simple closed curve $\gamma$ has slope $\sigma$. Thus, we have constructed $K$ and $\sigma$. If we wish, we can also calculate $\Delta(\lambda, \sigma)$ and $\Delta(\mu, \sigma)$. If $K$ is homologically trivial, this (together with orientation information) gives the rational number $p / q$ associated with $\sigma$.

The above algorithm constructs a finite number of possibilities for $K$ and $\sigma$. We now wish to rule out the cases where $K$ and $\sigma$ are neither exceptional nor norm-exceptional. We construct the manifold $M_{K}(\sigma)$. There is an algorithm to determine whether $M_{K}(\sigma)$ is reducible ([5] and [11]), and there is an algorithm to determine whether $M_{K}(\sigma)$ is a solid torus ([5] and [11]). The assumption that $H_{2}(M-\operatorname{int}(\mathcal{N}(K)), \partial M)$ is nontrivial implies that $H_{1}\left(M_{K}(\sigma)\right)$ is infinite and hence that $\pi_{1}\left(M_{K}(\sigma)\right)$ is infinite. If $M_{K}(\sigma)$ is irreducible, then according to Corollary 9.9 of $[3], \pi_{1}\left(M_{K}(\sigma)\right)$ is torsionfree. Thus, if the core of the surgery solid torus in $M_{K}(\sigma)$ has finite order in $\pi_{1}\left(M_{K}(\sigma)\right)$, then it is homotopically trivial. There is an algorithm to determine this, since the word problem is soluble for the fundamental groups of Haken 3-manifolds [13]. Finally, there is an algorithm to find the unit ball of the Thurston norm (Algorithm 5.9 of [12]), and so we can determine whether $\sigma$ is norm-exceptional.

\section{The sutured manifold theory background.}

The definition of an exceptional surgery was specifically designed so that sutured manifold theory can be applied. Sutured manifolds were defined 
and studied by Gabai [1] who used them to construct taut foliations on certain 3-manifolds. In this section, we will outline a version of the theory due to Scharlemann [10]. Almost everything in this section can be found elsewhere, mostly in Scharlemann's paper [10]. We include it here because it is absolutely central to our argument, but a reader familiar with the theory of sutured manifolds may safely skip this section.

Sutured manifold theory is intimately linked to the Thurston norm. Here, we give a definition of the Thurston norm and some related definitions of tautness.

Let $S$ be a compact oriented surface embedded in a compact oriented 3-manifold $M$. Let $\chi(S)$ denote its Euler characteristic. If $S$ is connected, define

$$
\chi_{-}(S)=\max \{0,-\chi(S)\} .
$$

When $S$ is not connected, define $\chi_{-}(S)$ to be the sum of $\chi_{-}\left(S_{i}\right)$ over all the components $S_{i}$ of $S$.

Let $P$ be a subset of $\partial M$, and let $z$ be an element of $H_{2}(M, P)$ which is represented by some embedded compact oriented surface. Then the Thurston norm of $z$ is given by

$$
x(z)=\min \left\{\chi_{-}(S): S \text { is an embedded surface representing } z\right\} .
$$

Let $(S, \partial S) \subset(M, \partial M)$ be an oriented compact surface embedded in $M$. Let $P$ be a subset of $\partial M$ which contains $\partial S$. Then $S$ is norm-minimising in $H_{2}(M, P)$ if $x([S, \partial S])=\chi_{-}(S)$. In the case where $P=\partial S$, then $S$ is taut if it is incompressible and norm minimising in $H_{2}(M, P)$. This use of the word 'taut' is not entirely standard; some authors (for example, [12]) insist only that $S$ be incompressible and norm-minimising in its class in $H_{2}(M, \partial M)$. However, our definition is more suited to sutured manifold theory.

A sutured manifold $(M, \gamma)$ is a compact oriented 3-manifold $M$, with $\partial M$ decomposed into two subsurfaces $\mathcal{R}_{-}$and $\mathcal{R}_{+}$, such that $\mathcal{R}_{-} \cup \mathcal{R}_{+}=\partial M$ and $\mathcal{R}_{-} \cap \mathcal{R}_{+}=\gamma$, where $\gamma$ is a union of disjoint simple closed curves, known as the sutures. The subsurfaces $\mathcal{R}_{-}$and $\mathcal{R}_{+}$are oriented so that the normal vectors of $\mathcal{R}_{-}$(respectively, $\mathcal{R}_{+}$) point into (respectively, out of) $M$. The symbol $\mathcal{R}_{ \pm}$will denote ' $\mathcal{R}_{-}$or $\mathcal{R}_{+}$'. When we wish to emphasise a particular sutured manifold, we will use the symbol $\mathcal{R}_{ \pm}(M)$.

A sutured manifold $(M, \gamma)$ is taut if $M$ is irreducible, and $\mathcal{R}_{-}$and $\mathcal{R}_{+}$are taut. For example it is not hard to show the following. Suppose that $\partial M$ is a (possibly empty) union of tori, and that $\mathcal{R}_{-}=\partial M$ and $\mathcal{R}_{+}=\emptyset$. Then $(M, \emptyset)$ is taut if and only is $M$ is neither reducible nor a solid torus.

One of the main techniques of the theory is to decompose a sutured manifold along a properly embedded surface. If $(M, \gamma)$ is a sutured manifold, and $S$ is an oriented surface properly embedded in $M$, with $\partial S$ and $\gamma$ in general position, then $M_{S}=M-\operatorname{int}(\mathcal{N}(S))$ inherits a sutured manifold structure 
$\left(M_{S}, \gamma_{S}\right)$. This is written $(M, \gamma) \stackrel{S}{\longrightarrow}\left(M_{S}, \gamma_{S}\right)$. This decomposition is said to be taut if $(M, \gamma)$ and $\left(M_{S}, \gamma_{S}\right)$ are both taut.

If $(M, \gamma)$ is a connected taut sutured manifold and $z$ is any nonzero homology class in $H_{2}(M, \partial M)$, then (Theorem 2.6 of $[\mathbf{1 0}]$ ) there is a taut decomposition $(M, \gamma) \stackrel{S}{\longrightarrow}\left(M_{S}, \gamma_{S}\right)$ such that:

(i) No curve of $\partial S$ bounds a disc in $\mathcal{R}_{ \pm}(M)$,

(ii) no component $X$ of $M_{S}$ has $\partial X \subset \mathcal{R}_{-}\left(M_{S}\right)$ or $\partial X \subset \mathcal{R}_{+}\left(M_{S}\right)$, and (iii) $[S, \partial S]=z \in H_{2}(M, \partial M)$.

When $S$ satisfies (i), we will say that $\partial S$ has essential intersection with $\mathcal{R}_{ \pm}(M)$. It is not hard to show that if $(M, \gamma) \stackrel{S}{\longrightarrow}\left(M_{S}, \gamma_{S}\right)$ is a taut decomposition and $\partial S$ has essential intersection with $\mathcal{R}_{ \pm}(M)$, then $S$ itself is taut.

Thus if $H_{2}(M, \partial M) \neq 0$, we may perform a taut sutured manifold decomposition along a taut surface having essential intersection with $\mathcal{R}_{ \pm}$. But if $H_{2}(M, \partial M)$ is trivial, then it is a classical fact that $\partial M$ is a (possibly empty) union of 2 -spheres. If $M$ is irreducible, then this implies that either $\partial M=\emptyset$ or $M$ is a collection of 3-balls. Using this argument, Gabai proved that, if $(M, \gamma)$ is a connected taut sutured manifold and $H_{2}(M, \partial M) \neq 0$, then there is a sequence of taut decompositions

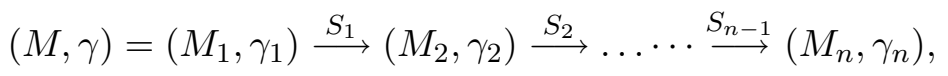

with $M_{n}$ a union of 3-balls.

An important step in Gabai's argument is to show that this sequence of decompositions cannot continue indefinitely. This is not at all obvious. In the case where $S_{i}$ is incompressible and $\partial$-incompressible in $M_{i}$ and has no component parallel to a subsurface of $\partial M_{i}$, it was proved by Haken [2] that such a sequence of decompositions must eventually terminate. However, it is sometimes necessary to consider surfaces $S_{i}$ which are $\partial$-compressible. Nevertheless, Gabai constructed a (complicated) argument which proved that this sequence of taut sutured manifold decompositions can be guaranteed to terminate. He did this by defining a complexity of a sutured manifold and then arguing by induction. In Section 5, we will offer a new definition of complexity for a sutured manifold with a given handle decomposition.

There is an extremely useful property of sutured manifold decompositions, which is summarised in the phrase 'tautness usually pulls back'. It is this property which makes sutured manifold theory distinctly different from the theory of Haken manifolds.

Theorem 3.1 (Theorem 3.6 of $[\mathbf{1 0}])$. Let $(M, \gamma) \stackrel{S}{\longrightarrow}\left(M_{S}, \gamma_{S}\right)$ be a decomposition, where $\partial S$ has essential intersection with $\mathcal{R}_{ \pm}(M)$, and where no component of $S$ is a compression disc for a torus component of $\mathcal{R}_{ \pm}(M)$. If $\left(M_{S}, \gamma_{S}\right)$ is taut, then so is $(M, \gamma)$. 
There is a partial converse to this theorem which can be useful. If $D$ is a disc properly embedded in $M$ intersecting $\gamma$ transversely in two points, then $D$ is known as a product disc. If $A$ is an annulus properly embedded in $M$ with one component of $\partial A$ in $\mathcal{R}_{-}$and one in $\mathcal{R}_{+}$, then $A$ is known as a product annulus. These surfaces play a useful rôle, since if $(M, \gamma) \stackrel{S}{\longrightarrow}\left(M_{S}, \gamma_{S}\right)$ is a decomposition along a product disc or an incompressible product annulus, then $(M, \gamma)$ is taut if and only if $\left(M_{S}, \gamma_{S}\right)$ is taut.

We have now described enough sutured manifold theory to explain the definition of an exceptional surgery curve, given in Section 1. The following argument is well-known, and is due to Gabai [1]. Let $K$ be a knot in a compact connected orientable 3 -manifold $M$, where $\partial M$ is (possibly empty) union of tori. If $M-\operatorname{int}(\mathcal{N}(K))$ is neither reducible nor a solid torus, then $(M-\operatorname{int}(\mathcal{N}(K)), \emptyset)$ is a taut sutured manifold, with $\mathcal{R}_{-}=\partial M \cup \partial \mathcal{N}(K)$. If $H_{2}(M-\operatorname{int}(\mathcal{N}(K)), \partial M) \neq 0$, then we may perform a taut sutured manifold decomposition

$$
(M-\operatorname{int}(\mathcal{N}(K))) \stackrel{S_{1}}{\longrightarrow}\left(M_{2}-\operatorname{int}(\mathcal{N}(K))\right),
$$

such that:

- $S_{1}$ is disjoint from $\partial \mathcal{N}(K)$,

- no simple closed curve of $\partial S_{1}$ bounds a disc in $\mathcal{R}_{ \pm}(M)$, and

- no component $X$ of $M_{2}$ has $\partial X \subset \mathcal{R}_{-}\left(M_{2}\right)$ or $\partial X \subset \mathcal{R}_{+}\left(M_{2}\right)$.

If $K$ is norm-exceptional, we insist that $\left[S_{1}, \partial S_{1}\right]=z \in H_{2}(M-\operatorname{int}(\mathcal{N}(K))$, $\partial M)$, where $z$ is the relevant homology class from Definition 1.3. Repeating this process, we construct a sequence of taut sutured manifold decompositions

$$
(M-\operatorname{int}(\mathcal{N}(K)), \emptyset) \stackrel{S_{1}}{\longrightarrow} \cdots \stackrel{S_{n-1}}{\longrightarrow}\left(M_{n}-\operatorname{int}(\mathcal{N}(K)), \gamma_{n}\right),
$$

satisfying the above conditions, and where $H_{2}\left(M_{n}-\operatorname{int}(\mathcal{N}(K)), \partial M_{n}\right)=0$. If $M-\operatorname{int}(\mathcal{N}(K))$ is atoroidal, then it is possible to show that this implies that $M_{n}$ is a solid torus regular neighbourhood of $K$ and possibly some 3-balls. No component $X$ of $M_{n}$ has $\partial X \subset \mathcal{R}_{-}\left(M_{n}\right)$ or $\partial X \subset \mathcal{R}_{+}\left(M_{n}\right)$. In particular, if $X$ is the component of $M_{n}$ containing $K$, then $\gamma_{n} \cap X$ is a collection of essential curves on $\partial X$, parallel to some slope $\rho$, say, on $\partial \mathcal{N}(K)$. If we Dehn fill $M-\operatorname{int}(\mathcal{N}(K))$ via any slope $\tau$ on $\partial \mathcal{N}(K)$, then $M_{n}-\operatorname{int}(\mathcal{N}(K))$ is filled to become a 3-manifold $M_{n}(\tau)$ which is a solid torus and some 3 -balls. Now, $M_{n}(\tau)$ inherits a sutured manifold structure $\left(M_{n}(\tau), \gamma_{n}\right)$ from $M_{n}-\operatorname{int}(\mathcal{N}(K))$, which is taut if the surgery slope $\tau$ is not the slope $\rho$ of the sutures. Since tautness pulls back, this implies that

$$
\left(M_{K}(\tau), \emptyset\right) \stackrel{S_{1}}{\longrightarrow} \cdots \stackrel{S_{n-1}}{\longrightarrow}\left(M_{n}(\tau), \gamma_{n}\right)
$$

is a sequence of taut sutured manifolds, with each $S_{i}$ taut in $M_{i}(\tau)$. This implies that:

(i) $M_{K}(\tau)$ is irreducible, 
(ii) $M_{K}(\tau)$ is not a solid torus,

(iii) the core of the surgery solid torus in $M_{K}(\tau)$ has infinite order in $\pi_{1}\left(M_{K}(\tau)\right)$, and

(iv) $S_{1}$ is taut in $M_{K}(\tau)$.

Now, if $\sigma$ is an exceptional or norm-exceptional surgery slope on $\partial \mathcal{N}(K)$, then at least one of the above cannot be true for $M_{K}(\sigma)$. Thus, $\sigma$ must be the slope $\rho$ which is parallel to the sutures in $M_{n}$. We assume in Theorems 1.4, 1.5 and 1.6 that $\Delta(\sigma, \mu)>1$ which implies in particular that $\sigma \neq \mu$. Thus, the facts (i)-(iv) above are true for $\tau=\mu$, and also

$$
(M, \emptyset) \stackrel{S_{1}}{\longrightarrow} \cdots \stackrel{S_{n-1}}{\longrightarrow}\left(M_{n}, \gamma_{n}\right)
$$

is a taut sutured manifold sequence. Each component of $\gamma_{n}$ lies inside $M$ as the $\sigma$-cable of $K$, or as an unknotted curve. The idea behind Theorems 1.4, 1.5 and 1.6 is (roughly speaking) inductively to find nice embeddings of $M_{i}$ in $M$. In particular, we will show that we can arrange that $\gamma_{n} \cap \Delta^{3}$ is one of a finite list of possibilities for each 3-simplex $\Delta^{3}$ of $M$. Since one component of $\gamma_{n}$ is the $\sigma$-cable of $K$, this will establish Theorem 1.5.

Thus, our definition of an exceptional surgery curve fits neatly into the sutured manifold setting. The sutured manifold theory which we have outlined above formed the basis for a theorem in [6] which will be an important technical tool in this paper. This result (Theorem 1.4 of [6]) deals with the interaction of exceptional surgery curves and embedded surfaces in a sutured manifold, and is given below.

Theorem 3.2. Let $(M, \gamma)$ be a taut sutured manifold, let $K$ be a knot in $M$ and let $\sigma$ be a slope on $\partial \mathcal{N}(K)$. Suppose that at least one of the following is true:

(i) $\sigma$ is an exceptional surgery slope, or

(ii) $\sigma$ is a norm-exceptional surgery slope, $\partial M$ is a (possibly empty) union of tori and $\gamma=\emptyset$.

Suppose that $\Delta(\sigma, \mu)>1$, where $\mu$ is the meridian slope on $\partial \mathcal{N}(K)$. Suppose also that $M-\operatorname{int}(\mathcal{N}(K))$ is irreducible and atoroidal and that $\mathrm{H}_{2}(M-$ $\operatorname{int}(\mathcal{N}(K)), \partial M) \neq 0$. Let $F$ be a surface properly embedded in $M$, with components $F_{1}, \ldots, F_{n}$, none of which is a sphere or disc disjoint from $\gamma$. Then there is an ambient isotopy of $K$ in $M$, after which we have the following inequality for each $i$ :

$$
\left|K \cap F_{i}\right| \leq \frac{-2 \chi\left(F_{i}\right)+\left|\gamma \cap F_{i}\right|}{2(\Delta(\sigma, \mu)-1)} .
$$

The numerator $-2 \chi\left(F_{i}\right)+\left|\gamma \cap F_{i}\right|$ is known as the index $I\left(F_{i}\right)$ of $F_{i}$. Note in particular that a product disc and an annulus disjoint from $\gamma$ both have zero index. Thus, if $\Delta(\sigma, \mu)>1$, Theorem 3.2 implies that we may ambient isotope $K$ off a collection of product discs and annuli disjoint from 
$\gamma$. Given that such surfaces play a useful technical rôle in sutured manifold theory, this will be very convenient. In fact, this is the only point in proof of Theorems 1.4, 1.5 and 1.6 where we use that $\Delta(\sigma, \mu)>1$.

\section{Vertical form and standard form for submanifolds.}

Recall that we are given a generalised triangulation of $M$. From this, we will construct the dual handle decomposition, which associates an $i$-handle with each $(3-i)$-simplex of $M$ not lying entirely in $\partial M$. For this dual handle decomposition, the boundary of each 0-handle has at most four discs of intersection with the 1-handles, and each 1-handle has at most three discs of intersection with the 2-handles. An example is given below.

We will now give some definitions and conventions regarding handle decompositions. We will throughout this paper denote the $i$-handles of a handle decomposition by $\mathcal{H}^{i}$. Henceforth, we will only consider handle decompositions of $n$-manifolds with the following properties:

- For $i>0$, the $i$-handles are attached to $\bigcup_{j<i} \mathcal{H}^{j}$.

- If $H_{i}=D^{n-i} \times D^{i}$ (respectively, $H_{j}=D^{n-j} \times D^{j}$ ) is an $i$-handle (respectively, $j$-handle) with $j<i$, then $H_{i} \cap H_{j}=E \times D^{j}=D^{n-i} \times F$ for some submanifold $E$ (respectively, $F$ ) of $\partial D^{n-j}$ (respectively, $\partial D^{i}$ ).

In words, the second condition requires that the attaching map of each handle respects the product structures of the handles to which it is attached. For a 3-manifold, this is relevant only for $j=1$ and $i=2$. In the case of a handle decomposition of a 3 -manifold, we also insist that:

- No 2-handle is disjoint from $\mathcal{H}^{1}$.

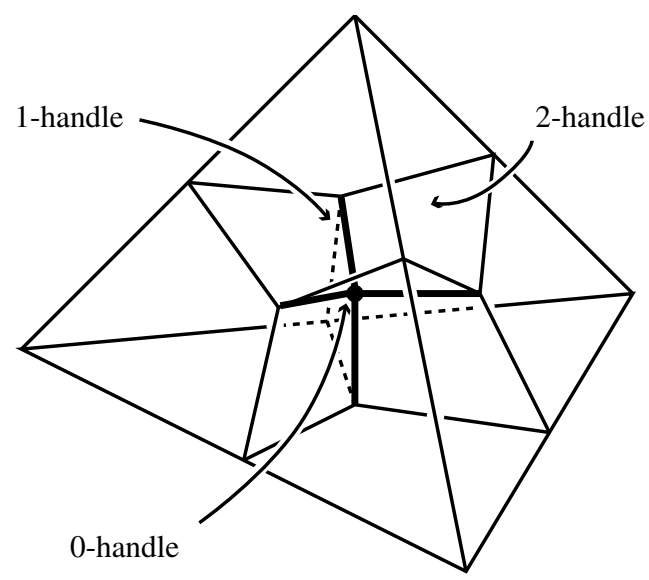

Figure 4.1. 
We will use the term handle structure for a decomposition satisfying these conditions. Note in particular that the dual handle decomposition of a 3manifold arising from a generalised triangulation has these properties. We will use $\mathcal{H}$ to denote a handle structure, but occasionally, we will also write $\mathcal{H}(M)$ when we wish to emphasise the manifold $M$. Note also that a handle structure $\mathcal{H}$ on a 3 -manifold $M$ induces a handle structure on $\partial M$, which we will usually write as $\mathcal{H}(\partial M)$.

We will fix a handle structure $\mathcal{H}$ of $M$, and then will consider embedded submanifolds of $M$. We wish to ensure that each submanifold lies inside $\mathcal{H}$ in a manageable way. The relevant notions are 'vertical' and 'standard' form, the first of which we now define.

Definition 4.2. Let $M$ be an $n$-manifold with a handle structure $\mathcal{H}$. Let $S$ be an $(n-1)$-manifold properly embedded in $M$. Then $S$ is in vertical form if, for each $i$-handle $D^{n-i} \times D^{i}$ of $\mathcal{H}$, we have $S \cap\left(D^{n-i} \times D^{i}\right)=E \times D^{i}$, where $E$ is a properly embedded submanifold of $D^{n-i}$. In particular, $S$ is disjoint from $\mathcal{H}^{n}$.

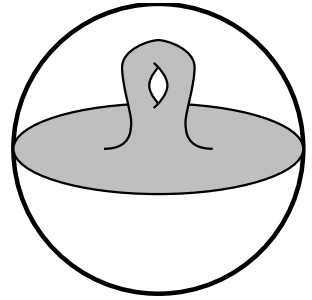

0-handle

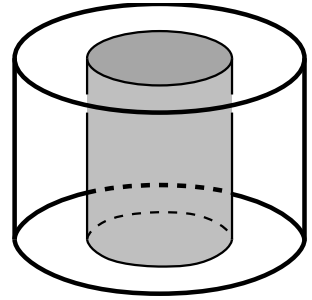

1-handle

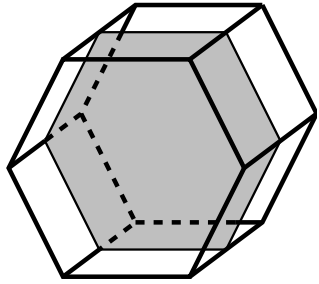

2-handle

Figure 4.3.

The only two cases which we will consider are where $n=2$ or $n=3$. Examples of 2-manifolds in vertical form in a 3-manifold are given in Fig. 4.3. The relevance of vertical form is its ubiquity.

Lemma 4.4. Let $M$ be an $n$-manifold with a handle structure $\mathcal{H}$, and let $S$ be an $(n-1)$-manifold properly embedded in $M$. Then there is an ambient isotopy which takes $S$ into vertical form with respect to $\mathcal{H}$.

Proof. We perform a sequence of ambient isotopies. The first pulls $S$ off $\mathcal{H}^{n}$. The second places $S$ in vertical form in $\mathcal{H}^{n-1}$, and so on. Let $C_{i}$ be the cocores of $\mathcal{H}^{i}$; thus $\mathcal{H}^{i}=C_{i} \times D^{i}$. We perform an ambient isotopy which makes $S$ transverse to $C_{i}$. By construction, $S$ is already vertical in $\mathcal{H}^{j}$ for $j>i$, and so we may take this isotopy to be supported in $\mathcal{H}^{i}-\partial \mathcal{H}^{i}$. After the isotopy, we may find a small disc $D_{0}^{i} \subset \operatorname{int}\left(D^{i}\right)$, such that $S \cap\left(C_{i} \times D_{0}^{i}\right)=E_{i} \times D_{0}^{i}$, 
for some submanifold $E_{i}$ of $C_{i}$. Then we may use the product structure on $D^{i}-\operatorname{int}\left(D_{0}^{i}\right) \cong S^{i-1} \times I$ to ambient isotope $C_{i} \times D_{0}^{i}$ onto $C_{i} \times D^{i}=\mathcal{H}^{i}$. We can take this isotopy of $M$ to be supported in an arbitrarily small neighbourhood of $\mathcal{H}^{i}$, and also to leave $S \cap \mathcal{H}^{j}$ invariant for $j>i$. After performing these isotopies for $i=n, n-1, \ldots, 0$, we finish with $S$ in vertical form.

If $(M, \gamma)$ is a sutured manifold with a handle structure $\mathcal{H}$, then by Lemma 4.4 there is an isotopy of $\partial M$ which takes $\gamma$ into vertical form (with respect to the induced handle decomposition $\mathcal{H}(\partial M)$ on $\partial M$ ). This isotopy of $\partial M$ extends to an isotopy of $M$. We can therefore assume that $\gamma$ is in vertical form in $\mathcal{H}(\partial M)$, and we will henceforth make this assumption.

If $S$ is any surface properly embedded in $M$, we would like to ensure that we can place $S$ in vertical form, and still keep $\gamma$ vertical in $\mathcal{H}(\partial M)$. This is the purpose of the following lemma.

Lemma 4.5. Let $(M, \gamma)$ be a sutured manifold with a handle structure $\mathcal{H}$, such that $\gamma$ is vertical in $\mathcal{H}(\partial M)$. If $S$ is a surface properly embedded in $M$, in general position with respect to $\gamma$, then there is an ambient isotopy which leaves $\gamma$ invariant and which moves $S$ into vertical form.

Proof. The first two steps of the ambient isotopy in Lemma 4.4 are supported in a small neighbourhood of $\mathcal{H}^{3} \cup \mathcal{H}^{2}$. Hence, we may assume that it leaves $\gamma$ fixed. Since $S$ and $\gamma$ are in general position, we may pick the co-cores $C_{1}$ of the 1-handles so that $C_{1} \cap S \cap \gamma=\emptyset$. The ambient isotopy supported in a neighbourhood of $\mathcal{H}^{1}$ can then be taken to leave $\gamma$ invariant. There is no restriction on $S \cap \mathcal{H}^{0}$, once $S$ lies in the remaining handles in the correct way. Hence, we have ambient isotoped $S$ into vertical form, leaving $\gamma$ invariant.

For inductive purposes, we define a notion of complexity for surfaces in vertical form in a handle structure of a 3-manifold.

Definition 4.6. The complexity of a vertical surface $S$ is the ordered pair of integers $\left(\left|S \cap \mathcal{H}_{2}\right|,\left|\partial S \cap \mathcal{H}_{1}\right|\right)$.

We order these pairs lexicographically. In other words, the pairs $\left(n_{1}, n_{2}\right)$ and $\left(m_{1}, m_{2}\right)$ satisfy $\left(n_{1}, n_{2}\right)>\left(m_{1}, m_{2}\right)$ precisely when:

- $n_{1}>m_{1}$, or

- $n_{1}=m_{1}$ and $n_{2}>m_{2}$.

It is clear that this ordering is a well-ordering.

In the case of surfaces in 3-manifolds, there is a notion which is a little stronger than vertical form.

Definition 4.7. Let $S$ be a vertical surface in a handle structure $\mathcal{H}$ of a 3-manifold $M$. Then $S$ is standard if $S$ intersects each handle of $\mathcal{H}$ in a (possibly empty) collection of discs. 


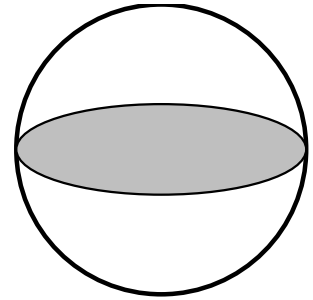

0-handle

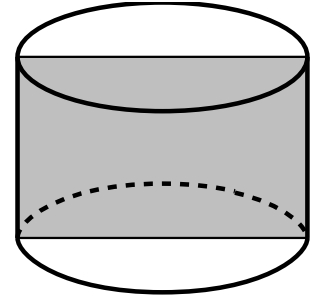

1-handle

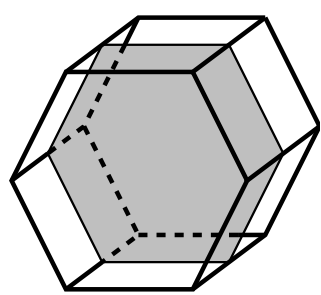

2-handle

Figure 4.8.

Examples of surfaces in standard form are given in Fig. 4.8. A general surface $S$ in $M$ might not have a representation in standard form, but if $S$ is incompressible and $M$ is irreducible, then we now show that it can be ambient isotoped into standard form.

Lemma 4.9. Let $(M, \gamma)$ be an irreducible sutured manifold with a handle structure $\mathcal{H}$. Let $S$ be a vertical incompressible surface properly embedded in $M$, with no component of $S$ a 2-sphere. Then there is an ambient isotopy of $S$ which leaves $\gamma$ fixed and which takes $S$ into standard form without increasing its complexity.

Proof. If $S$ is not in standard form, then it must differ from standard form in some 1-handle or some 0-handle of $\mathcal{H}$. Suppose first that, in some 1-handle $H_{1}=D^{2} \times D^{1}$, there is a component of $S \cap H_{1}$ which is $\alpha \times D^{1}$, for a simple closed curve $\alpha$. If both curves of $\alpha \times \partial D^{1}$ bound discs in $\mathcal{H}^{0}$, then $S$ has a 2 -sphere component. Hence, we may assume that $S$ differs from standard form in some 0-handle $H_{0}$. That is, suppose that $S \cap H_{0}$ is not a union of discs. Then, since no component of $S$ is a 2-sphere, $S \cap H_{0}$ is compressible in $H_{0}$, via a compression disc $D$. Since $S$ is incompressible, $\partial D$ bounds a disc $D^{\prime}$ in $S$. The disc $D^{\prime}$ does not lie wholly in $H_{0}$, and so must intersect $\mathcal{H}^{1}$. As $M$ is irreducible, we may ambient isotope $S$, taking $D^{\prime}$ onto $D$. This does not increase the complexity of $S$, and it reduces the number of components of $S \cap \mathcal{H}^{1}$. Hence, this process terminates with $S$ in standard form. The isotopy at each stage leaves $\partial M$ (and hence $\gamma$ ) fixed.

We may therefore assume that if $S$ and $M$ satisfy the conditions of Lemma 4.9, then $S$ is in standard form. We will now show that, if $\left(M_{S}, \gamma_{S}\right)$ is the sutured manifold resulting from the decomposition along $S$, then $M_{S}$ has an induced handle structure with $\gamma_{S}$ in vertical form in $\mathcal{H}\left(\partial M_{S}\right)$.

If $H$ is an $i$-handle $D^{3-i} \times D^{i}$ of $\mathcal{H}(M)$, then each component of $H-$ $\operatorname{int}(\mathcal{N}(S))$ inherits a structure $X \times D^{i}$, where $X$ is a $(3-i)$-submanifold of $D^{3-i}$. This is true because $S$ is vertical. Since $S$ is standard, then each component of $X$ is a copy of $D^{3-i}$, and so each component of $H-\operatorname{int}(\mathcal{N}(S))$ has the structure of an $i$-handle. These handles combine to give a handle 
structure on $M_{S}$. The curves $\gamma_{S}$ are a subgraph of the graph $\partial \mathcal{N}(\partial S) \cup \gamma$. Since $\partial S$ and $\gamma$ are both vertical in $\mathcal{H}(\partial M)$, the curves $\gamma_{S}$ are then vertical in $\mathcal{H}\left(\partial M_{S}\right)$.

It is a very useful property that $\left(M_{S}, \gamma_{S}\right)$ inherits a handle structure from that of $(M, \gamma)$. It is the basis for an inductive proof of Theorems 1.4, 1.5 and 1.6. However, to construct such a proof, we need to define a 'complexity' for handle structures.

\section{Complexity of handle structures of sutured manifolds.}

We will now define a notion of complexity for a handle structure $\mathcal{H}$ of a sutured manifold $(M, \gamma)$. We will focus on the 2 -spheres $\partial \mathcal{H}^{0}$. Lying in these 2-spheres, there is the surface $\partial \mathcal{H}^{0} \cap\left(\mathcal{H}^{1} \cup \mathcal{H}^{2}\right)$. We denote this surface by $\mathcal{F}(\mathcal{H})$, or sometimes simply $\mathcal{F}$.

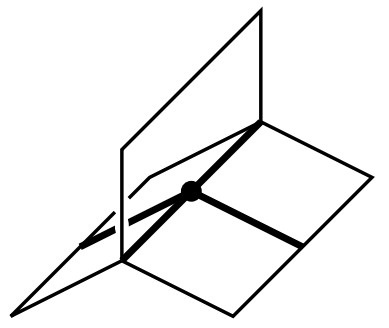

Picture of $M$ near 0-handle

$$
\downarrow
$$

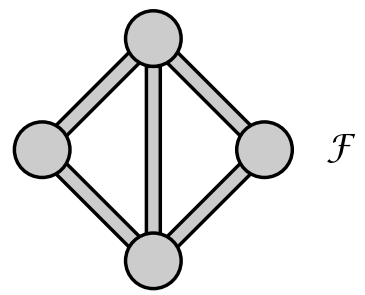

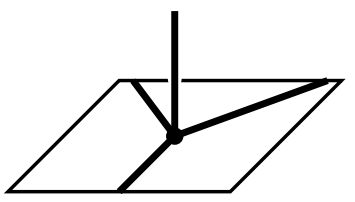

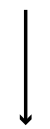

$\mathcal{F}$

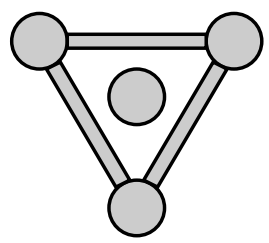

Figure 5.1.

Recall from Section 4 that we insisted that no 2-handle of $\mathcal{H}$ is disjoint from $\mathcal{H}^{1}$. Therefore, $\mathcal{F}$ inherits a handle structure, with $\partial \mathcal{H}^{0} \cap \mathcal{H}^{1}$ forming the 0 -handles of $\mathcal{F}$ (which we denote by $\mathcal{F}^{0}$ ), and $\partial \mathcal{H}^{0} \cap \mathcal{H}^{2}$ forming the 1-handles of $\mathcal{F}$ (which we denote by $\mathcal{F}^{1}$ ). Note that each component of the surface $\operatorname{cl}\left(\partial \mathcal{H}^{0}-\mathcal{F}\right)$ lies either in $\partial M$ or in $\partial \mathcal{H}^{3}$, and the curves $\gamma \cap \partial \mathcal{H}^{0}$ are properly embedded in $\operatorname{cl}\left(\partial \mathcal{H}^{0}-\mathcal{F}\right)$.

If $S$ is in standard form, then the simple closed curves $S \cap \partial \mathcal{H}^{0}$ satisfy the following (fairly weak) restrictions:

- $S \cap \partial \mathcal{H}^{0}$ is disjoint from $\mathcal{H}^{3}$, 
- $S \cap \mathcal{F}$ is vertical in $\mathcal{F}$,

- no curve of $S \cap \partial \mathcal{H}^{0}$ lies entirely within a 0 -handle of $\mathcal{F}$.

The nature of $\mathcal{F}$ will determine the complexity of $\mathcal{H}$. One invariant of $\mathcal{F}$ will be of particular importance, namely its index. Recall from Section 3 that the index $I(F)$ of a component $F$ of $\mathcal{F}$ is defined to be

$$
I(F)=-2 \chi(F)+|F \cap \gamma| \text {. }
$$

If $V$ is a 0 -handle of $\mathcal{F}$, then the valence of $V$ is the number of $\operatorname{arcs}$ of $V \cap \mathcal{F}^{1}$. We also define the index of $V$ to be

$$
I(V)=\left|V \cap \mathcal{F}^{1}\right|+|V \cap \gamma|-2 .
$$

The reason for this terminology is that

$$
I(F)=\sum_{V \in F \cap \mathcal{F}^{0}} I(V) .
$$

For each component $F$ of $\mathcal{F}$, we define the following integers:

$$
\begin{aligned}
& C_{1}(F)=\left|F \cap \mathcal{F}^{1}\right|+1, \\
& C_{2}(F)=I(F), \\
& C_{3}(F)=|\partial F| .
\end{aligned}
$$

The $\mathcal{F}$-complexity set $C_{\mathcal{F}}(\mathcal{H})$ of $\mathcal{H}$ is defined to be the set of ordered triples

$$
C_{\mathcal{F}}(\mathcal{H})=\left\{\left(C_{1}(F), C_{2}(F), C_{3}(F)\right): F \text { a component of } \mathcal{F} \text { with } I(F)>0\right\},
$$

where repetitions are retained. If $X$ is a subset of $M$, with $X \cap \mathcal{F}$ a nonempty collection of components of $\mathcal{F}$, then we similarly define

$$
C_{\mathcal{F}}(X)=\left\{\left(C_{1}(F), C_{2}(F), C_{3}(F)\right): F\right.
$$

a component of $X \cap \mathcal{F}$ with $I(F)>0\}$,

where again repetitions are retained.

An example is given in Fig. 5.2 of how $\mathcal{F}$ and its complexity behave when $\mathcal{H}$ is decomposed along a surface $S$.

We compare the triples $\left(C_{1}(F), C_{2}(F), C_{3}(F)\right)$ and $\left(C_{1}\left(F^{\prime}\right), C_{2}\left(F^{\prime}\right)\right.$, $\left.C_{3}\left(F^{\prime}\right)\right)$ by defining $\left(C_{1}(F), C_{2}(F), C_{3}(F)\right)>\left(C_{1}\left(F^{\prime}\right), C_{2}\left(F^{\prime}\right), C_{3}\left(F^{\prime}\right)\right)$ if:

- $C_{1}(F)>C_{1}\left(F^{\prime}\right)$, or

- $C_{1}(F)=C_{1}\left(F^{\prime}\right)$ and $C_{2}(F)>C_{2}\left(F^{\prime}\right)$, or

- $C_{1}(F)=C_{1}\left(F^{\prime}\right)$ and $C_{2}(F)=C_{2}\left(F^{\prime}\right)$ and $C_{3}(F)>C_{3}\left(F^{\prime}\right)$.

It is clear that this is a total ordering and a well-ordering.

We define a total order on the $\mathcal{F}$-complexity of handle structures, as follows. If $\mathcal{H}$ and $\mathcal{H}^{\prime}$ are two handle structures, we order their $\mathcal{F}$-complexity sets $C_{\mathcal{F}}(\mathcal{H})$ and $C_{\mathcal{F}}\left(\mathcal{H}^{\prime}\right)$ into two non-increasing sequences of triples. We extend each of these sequences by concatenating with an infinite sequence of triples $(0,0,0)$. (Note that always $C_{1}(F)>0$, and so $\left(C_{1}(F), C_{2}(F), C_{3}(F)\right.$ ) $>(0,0,0)$.) Then, we compare the first (and hence largest) triple $\left(C_{1}(F)\right.$, 

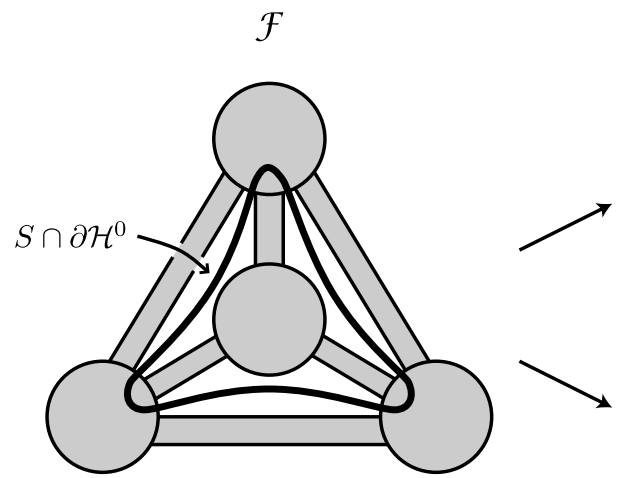

$\mathcal{F}$-complexity $=(7,4,4)$

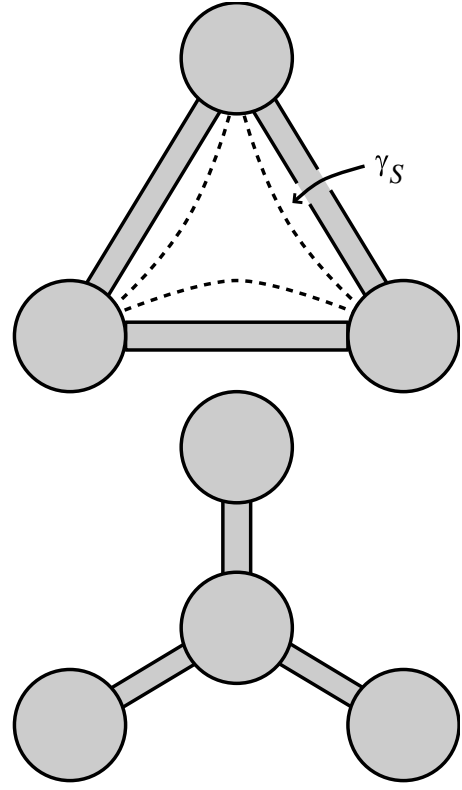

$\mathcal{F}$-complexity $=(4,6,2)$

Figure 5.2.

$\left.C_{2}(F), C_{3}(F)\right)$ of $C_{\mathcal{F}}(\mathcal{H})$ with the first (and hence largest) triple $\left(C_{1}\left(F^{\prime}\right)\right.$, $\left.C_{2}\left(F^{\prime}\right), C_{3}\left(F^{\prime}\right)\right)$ of $C_{\mathcal{F}}\left(\mathcal{H}^{\prime}\right)$. If $\left(C_{1}(F), C_{2}(F), C_{3}(F)\right)>\left(C_{1}\left(F^{\prime}\right), C_{2}\left(F^{\prime}\right)\right.$, $\left.C_{3}\left(F^{\prime}\right)\right)$, say, then we define $C_{\mathcal{F}}(\mathcal{H})>C_{\mathcal{F}}\left(\mathcal{H}^{\prime}\right)$. Otherwise, we pass to the second triples of $C_{\mathcal{F}}(\mathcal{H})$ and $C_{\mathcal{F}}\left(\mathcal{H}^{\prime}\right)$. Continuing in this way, we can compare the $\mathcal{F}$-complexities of $\mathcal{H}$ and $\mathcal{H}^{\prime}$.

We now define the complexity $C(\mathcal{H})$ of a handle structure $\mathcal{H}$ to be the ordered pair $\left(C_{\mathcal{F}}(\mathcal{H}), n(\mathcal{H})\right)$, where $n(\mathcal{H})$ is the number of 0 -handles of $\mathcal{H}$ containing a component of $\mathcal{F}(\mathcal{H})$ with positive index. We compare the complexity of handle structures $\mathcal{H}$ and $\mathcal{H}^{\prime}$ by asserting that $C(\mathcal{H})>C\left(\mathcal{H}^{\prime}\right)$ if one of the following holds:

- $C_{\mathcal{F}}(\mathcal{H})>C_{\mathcal{F}}\left(\mathcal{H}^{\prime}\right)$, or

- $C_{\mathcal{F}}(\mathcal{H})=C_{\mathcal{F}}\left(\mathcal{H}^{\prime}\right)$ and $n(\mathcal{H})<n\left(\mathcal{H}^{\prime}\right)$.

Lemma 5.3. This ordering on complexity of handle structures is a wellordering.

Proof. We need to show that there cannot exist an infinite strictly decreasing sequence $\left\{C\left(\mathcal{H}_{i}\right): i \in \mathbb{N}\right\}$. Suppose that there is such a sequence. Then $C_{\mathcal{F}}\left(\mathcal{H}_{i}\right) \geq C_{\mathcal{F}}\left(\mathcal{H}_{i+1}\right)$ for each $i$. Suppose first that this inequality is strict for only finitely many $i$. Then we may pass to a subsequence in which $C_{\mathcal{F}}\left(\mathcal{H}_{i}\right)$ is constant. Then the number of components of $\mathcal{F}\left(\mathcal{H}_{i}\right)$ with positive index 
is constant. However, since $C\left(\mathcal{H}_{i}\right)>C\left(\mathcal{H}_{i+1}\right)$ for each $i, n\left(\mathcal{H}_{i}\right)<n\left(\mathcal{H}_{i+1}\right)$ for each $i$. This is impossible.

Therefore, we may suppose that $C_{\mathcal{F}}\left(\mathcal{H}_{i}\right)>C_{\mathcal{F}}\left(\mathcal{H}_{i+1}\right)$ for infinitely many $i$. Pass to this subsequence. Let $T_{i}^{n}$ be the $n^{\text {th }}$ largest triple of $C_{\mathcal{F}}\left(\mathcal{H}_{i}\right)$. For each $i$, there is a natural number $N(i)$, such that:

- $T_{i}^{n}=T_{i+1}^{n}$ for $n<N(i)$, and

- $T_{i}^{N(i)}>T_{i+1}^{N(i)}$.

Define $M(i)=\min _{j \geq i} N(j)$. Then $\{M(i): i \in \mathbb{N}\}$ is a non-decreasing sequence. For all $i, M(i) \leq N(i)$, and for infinitely many $i$, this is an equality. Consider the sequence of triples $\left\{T_{i}^{M(i)}: i \in \mathbb{N}\right\}$. Then $T_{i}^{M(i)} \geq$ $T_{i+1}^{M(i)} \geq T_{i+1}^{M(i+1)}$. For the infinitely many $i$ when $M(i)=N(i)$, we have

$$
T_{i}^{M(i)}=T_{i}^{N(i)}>T_{i+1}^{N(i)}=T_{i+1}^{M(i)} \geq T_{i+1}^{M(i+1)} .
$$

Thus, the infinite sequence of triples $\left\{T_{i}^{M(i)}: i \in \mathbb{N}\right\}$ contains an infinite strictly decreasing sequence. This is impossible, since the ordering on the triples is a well-ordering.

By the above lemma, we can use the complexity of handle structures as the basis for an inductive argument. We will start with a sutured manifold $(M, \gamma)$ with a handle structure $\mathcal{H}$. If $H_{2}(M, \partial M) \neq 0$, we will perform a taut decomposition $(M, \gamma) \stackrel{S}{\longrightarrow}\left(M_{S}, \gamma_{S}\right)$. The manifold $M_{S}$ will inherit a handle structure $\mathcal{H}^{\prime}$. We will try to ensure that the complexity of $\mathcal{H}^{\prime}$ is no more than that of $\mathcal{H}$ (and preferably, strictly less than that of $\mathcal{H}$ ). The following lemma asserts that, to guarantee this, we need only restrict attention to smaller parts of $\mathcal{H}$. For example, it shows that we need only check $C\left(H_{0} \cap \mathcal{H}^{\prime}\right) \leq C\left(H_{0}\right)$ for each 0-handle $H_{0}$ of $\mathcal{H}$.

Lemma 5.4. Let $\mathcal{H}$ (respectively $\mathcal{H}^{\prime}$ ) be a handle structure for a sutured manifold $(M, \gamma)$ (respectively $\left(M^{\prime}, \gamma^{\prime}\right)$ ). Suppose that the 0 -handles of $\mathcal{H}$ (respectively $\mathcal{H}^{\prime}$ ) have been partitioned into $n$ subsets $A_{1}, \ldots, A_{n}$ (respectively, $\left.A_{1}^{\prime}, \ldots, A_{n}^{\prime}\right)$. (For example, each $A_{i}$ may be some 0 -handle $H_{0}$ of $\mathcal{H}$, and $A_{i}^{\prime}$ is $H_{0} \cap \mathcal{H}^{\prime}$.) Suppose that for each $i, C\left(A_{i}^{\prime}\right) \leq C\left(A_{i}\right)$. Then $C\left(\mathcal{H}^{\prime}\right) \leq$ $C(\mathcal{H})$. Additionally, if $C\left(A_{i}^{\prime}\right)<C\left(A_{i}\right)$ for some $i$, then $C\left(\mathcal{H}^{\prime}\right)<C(\mathcal{H})$.

Proof. Arrange the triples of $C_{\mathcal{F}}(\mathcal{H})$ into a non-increasing sequence $\left\{T_{j}\right.$ : $j \in \mathbb{N}\}$. Consider the first integer $j$ for which $T_{j}>T_{j+1}$. Then the triples $T_{1}, \ldots, T_{j}$ are all some fixed triple $T$. The partitioning of $\mathcal{H}^{0}$ gives a partitioning of $T_{1}, \ldots, T_{j}$ into $n$ subsets (some of which may be empty). Say that $k(i)$ of these lie in $A_{i}$. Since $C\left(A_{i}^{\prime}\right) \leq C\left(A_{i}\right)$, we must have $C_{\mathcal{F}}\left(A_{i}^{\prime}\right) \leq C_{\mathcal{F}}\left(A_{i}\right)$. So, there are at most $k(i)$ copies of $T$ in $A_{i}^{\prime}$, and there are no larger triples. Hence, in $C_{\mathcal{F}}\left(\mathcal{H}^{\prime}\right)$, there are at most $j$ copies of $T$ and no larger triples. If there are fewer than $j$ copies of $T$ in $C\left(\mathcal{H}^{\prime}\right)$, then $C_{\mathcal{F}}\left(\mathcal{H}^{\prime}\right)<C_{\mathcal{F}}(\mathcal{H})$, and the lemma is proved. Otherwise, we can remove 
each copy of $T$ from $C_{\mathcal{F}}(\mathcal{H})$ and $C_{\mathcal{F}}\left(\mathcal{H}^{\prime}\right)$, without affecting any ordering. Continuing in this fashion with the next largest triples of $C(\mathcal{H})$, and so on, we see that $C_{\mathcal{F}}\left(\mathcal{H}^{\prime}\right) \leq C_{\mathcal{F}}(\mathcal{H})$. Also, if we have equality, then we must have had $C_{\mathcal{F}}\left(A_{i}^{\prime}\right)=C_{\mathcal{F}}\left(A_{i}\right)$ for each $i$. Since $C\left(A_{i}^{\prime}\right) \leq C\left(A_{i}\right)$, the number of 0 -handles in $A_{i}^{\prime}$ containing components of $\mathcal{F}\left(\mathcal{H}^{\prime}\right)$ with positive index is at least the number of 0 -handles in $A_{i}$ containing components of $\mathcal{F}(\mathcal{H})$ with positive index. Therefore, $n\left(\mathcal{H}^{\prime}\right) \geq n(\mathcal{H})$ and so $C\left(\mathcal{H}^{\prime}\right) \leq C(\mathcal{H})$. Also, if we have equality, then we must have had $C\left(A_{i}^{\prime}\right)=C\left(A_{i}\right)$ for each $i$.

To perform an inductive argument we need to ensure that the complexity of $\mathcal{H}^{\prime}$ is less than that of $\mathcal{H}$, where $\mathcal{H}^{\prime}$ is the induced handle structure on $\left(M_{S}, \gamma_{S}\right)$. However, this is not in general true. To guarantee this, it is important that each 0 -handle of $\mathcal{F}$ has positive index, and to ensure this, we may first need to decompose $(M, \gamma)$ along some product discs and annuli, and then simplify the handle decomposition of the resulting sutured manifold. Even then, to ensure that complexity is reduced by decomposition along $S$, we may need to perform some modifications to $S$.

We will give these procedures in Sections 7-10. But first we explain the idea behind the above definition of complexity. The surface $S$ is in general $\partial$-compressible in $M$ and in [4] it was shown that there may exist infinitely long hierarchies of incompressible $\partial$-compressible surfaces in a 3 -manifold. Thus, it is vital that we use the fact that $M$ has a sutured manifold structure. This is encoded in the quantity $C_{2}(F)$ which was defined to be the index of a component $F$ of $\mathcal{F}$. We therefore study how index behaves under decomposition.

Let $\mathcal{H}$ (respectively, $\mathcal{H}^{\prime}$ ) be the handle decomposition of $(M, \gamma)$ (respectively, $\left.\left(M_{S}, \gamma_{S}\right)\right)$. Let $\mathcal{F}=\mathcal{F}(\mathcal{H})$ and let $\mathcal{F}^{\prime}=\mathcal{F}\left(\mathcal{H}^{\prime}\right)$. Let $V$ be a 0 -handle of $\mathcal{F}$ and let $V_{1}^{\prime}, \ldots, V_{k}^{\prime}$ be the 0 -handles $V \cap \mathcal{F}^{\prime}$. Now, $V_{1}^{\prime}, \ldots, V_{k}^{\prime}$ are obtained from $V$ by cutting along properly embedded arcs. The endpoint of each arc either lies in $\mathcal{R}_{ \pm}(M)$ or in $\mathcal{F}^{1}(\mathcal{H})$. Therefore, an elementary counting argument shows that

$$
I(V)=\sum_{i=1}^{k} I\left(V_{i}^{\prime}\right) .
$$

In particular, if $F$ is a component of $\mathcal{F}$ and $F^{\prime}=F \cap \mathcal{F}^{\prime}$, then $I(F)=I\left(F^{\prime}\right)$.

Hence, we can ensure that the quantity $C_{2}$ does not increase, as long as we create no discs of $\mathcal{F}^{\prime}$ with negative index. Thus, our goal is to alter $S$ in order to remove these discs. But, in general, this does not seem to be possible. An example is given in Figure 5.2. There, a 0-handle of $\mathcal{H}$ is decomposed into two 0-handles of $\mathcal{H}^{\prime}$. A negative index disc of $\mathcal{F}^{\prime}$ is created, but note that, nevertheless, the complexity of the handle structure has decreased. 
It is fairly easy to show that, in general, under mild assumptions on $S$, neither $C_{1}$ nor $C_{3}$ can increase. Our aim is to show that, if $C_{1}$ is left unchanged, then in fact no negative index discs of $\mathcal{F}^{\prime}$ are created, and so $C_{2}$ is not increased. Furthermore, if $C_{1}, C_{2}$ and $C_{3}$ are all left unchanged, then $[S, \partial S]=0 \in H_{2}(M, \partial M)$.

\section{Overview of the proof of the main theorems.}

We have now developed enough machinery to outline the proofs of Theorems 1.4, 1.5 and 1.6. We start with a generalised triangulation of $M$, and using this, we construct the dual handle structure $\mathcal{H}(M)$ (which we sometimes abbreviate to $\mathcal{H}$ ). Roughly speaking, the idea is to decompose $M$ along surfaces until we end with a solid torus neighbourhood of $K$, plus perhaps some 3-balls. At each stage, we will be examining a 3-manifold $M^{\prime}$ embedded in $M$. This manifold $M^{\prime}$ will have a handle structure which respects $\mathcal{H}(M)$, in the following sense.

Definition 6.1. Let $(M, \gamma)$ be a sutured manifold with a handle structure $\mathcal{H}(M)$. Let $\left(M^{\prime}, \gamma^{\prime}\right)$ be a sutured manifold lying in $M$ with a handle structure $\mathcal{H}\left(M^{\prime}\right)$. Then $\mathcal{H}\left(M^{\prime}\right)$ respects $\mathcal{H}(M)$ if each of the following conditions holds:

- The 0-handles of $M^{\prime}$ lie in the 0-handles of $M$.

- The 1-handles of $M^{\prime}$ lie in the 1-handles of $M$ in a vertical fashion and inherit their product structure.

- The surface $\mathcal{F}\left(M^{\prime}\right)$ lies in $\mathcal{F}(M)$, with the intersection $\mathcal{F}^{1}\left(M^{\prime}\right) \cap \mathcal{F}^{1}(M)$ lying in $\mathcal{F}^{1}(M)$ in a vertical fashion.

Note that, if $\mathcal{H}\left(M^{\prime}\right)$ respects $\mathcal{H}(M)$, then automatically the arcs $\gamma^{\prime} \cap$ $\mathcal{H}^{1}\left(M^{\prime}\right)$ are vertical in $\mathcal{H}^{1}(M)$ and the discs $\mathcal{H}^{2}\left(M^{\prime}\right) \cap \mathcal{H}^{1}\left(M^{\prime}\right)$ are vertical in $\mathcal{H}^{1}(M)$. Thus, the only restriction on the 2-handles of $M^{\prime}$ is a requirement on their attaching maps. The remainder of each 2-handle may lie inside $M$ in a complicated way.

Occasionally, the handle structure of $M^{\prime}$ will resemble the handle structure of $M$ in some 0-handle, in the following sense.

Definition 6.2. Suppose that the handle structure $\mathcal{H}\left(M^{\prime}\right)$ of $\left(M^{\prime}, \gamma^{\prime}\right)$ respects the handle structure $\mathcal{H}(M)$ of $(M, \gamma)$. Let $H_{0}$ be a 0 -handle of $M$. Then $\mathcal{H}\left(M^{\prime}\right) \cap H_{0}$ is obtained from $H_{0}$ by a trivial modification if each of the following conditions is satisfied:

(i) There is at most one 0-handle $H_{0}^{\prime}$ of $\mathcal{H}\left(M^{\prime}\right) \cap H_{0}$ containing a component of $\mathcal{F}\left(M^{\prime}\right)$ with positive index;

(ii) $H_{0}-H_{0}^{\prime}$ consists of a parallelity region $R$ between $\partial H_{0}-\partial H_{0}^{\prime}$ and $\partial H_{0}^{\prime}-\partial H_{0}$

(iii) for any component $F$ of $\mathcal{F}(M) \cap H_{0}$ with positive index, $F \cap R$ is either empty or a parallelity region between arcs and circles of $F \cap \partial H_{0}^{\prime}$ and 
arcs and circles in $\partial F$, the parallelity region respecting the handle structure of $F$;

(iv) the arcs $\partial H_{0}^{\prime} \cap \gamma^{\prime}$ are parallel in $R$ to the arcs of $\partial H_{0} \cap \gamma$, possibly joined up by index zero discs of $\mathcal{F}(M)$;

(v) $\partial H_{0}^{\prime} \cap \mathcal{F}(M)$ and $\partial H_{0}^{\prime} \cap \mathcal{F}\left(M^{\prime}\right)$ have the same handle structure.

Roughly speaking, a trivial modification leaves components of $\mathcal{F}(M)$ with positive index relatively unaltered.

We make the following definition: If $\mathcal{H}(M)$ is a handle structure for $M$, we define the important 0-handles $\mathcal{I} H^{0}(M)$ to be the 0 -handles $H_{0}$ with $H_{0} \cap \mathcal{F}$ containing at least one component with positive index. In Sections 7 and 8 , we will prove the following result, which gives a method of modifying a handle decomposition so that, afterwards, each 0 -handle of $\mathcal{F}$ has positive index. Recall from Section 5 that the index of a component of $\mathcal{F}$ is equal to the sum of the indices of its 0-handles. So this implies that each component of $\mathcal{F}$ has positive index. Therefore, each 0 -handle of $\mathcal{H}$ is either important or disjoint from the 1-handles and 2-handles.

Proposition 6.3. Let $\mathcal{H}(M)$ be a handle structure of a taut sutured manifold $(M, \gamma)$. Suppose that each component of $M$ has nonempty boundary, and that no component of $M$ is a solid torus. Suppose also that no component of $M$ is a Seifert fibre space disjoint from $\gamma$, with base space a disc and having two exceptional fibres. Then there is a (possibly empty) sequence of taut decompositions

$$
(M, \gamma) \stackrel{P_{1}}{\longrightarrow} \cdots \stackrel{P_{m}}{\longrightarrow}\left(M^{\prime}, \gamma^{\prime}\right),
$$

where each $P_{i}$ is either a product disc or an incompressible annulus disjoint from the sutures. There is a handle structure $\mathcal{H}\left(M^{\prime}\right)$ of $\left(M^{\prime}, \gamma^{\prime}\right)$ and an embedding of $M^{\prime}$ in $M$ isotopic to the embedding arising from the sutured manifold decomposition, with the following properties:

(i) $\mathcal{H}\left(M^{\prime}\right)$ respects $\mathcal{H}(M)$.

(ii) For each 0-handle $H_{0}$ of $\mathcal{H}(M)$, the complexity of $H_{0} \cap \mathcal{H}\left(M^{\prime}\right)$ is no more than that of $H_{0}$.

(iii) For each 0-handle $H_{0}$ of $\mathcal{H}(M)$, the intersections

$$
\begin{aligned}
& H_{0} \cap \mathcal{I} H^{0}\left(M^{\prime}\right) \\
& H_{0} \cap \mathcal{I} H^{0}\left(M^{\prime}\right) \cap \mathcal{F}\left(M^{\prime}\right) \\
& H_{0} \cap \mathcal{I} H^{0}\left(M^{\prime}\right) \cap \gamma^{\prime}
\end{aligned}
$$

are each one of a finite number of possibilities (up to trivial modifications), which depend only on $\mathcal{F}(M) \cap H_{0}$ and $H_{0} \cap \gamma$, and are otherwise independent of $M$ and $M^{\prime}$. 
(iv) If $H_{0}$ is a 0-handle of $\mathcal{H}(M)$ and the complexity of $H_{0} \cap \mathcal{H}\left(M^{\prime}\right)$ is equal to that of $H_{0}$, then $H_{0} \cap \mathcal{H}\left(M^{\prime}\right)$ is obtained from $H_{0}$ by a trivial modification.

(v) Each 0-handle of $\mathcal{F}\left(M^{\prime}\right)$ has positive index.

(vi) For each 0-handle $H_{0}^{\prime}$ of $\mathcal{H}\left(M^{\prime}\right), H_{0}^{\prime} \cap\left(\mathcal{F}\left(M^{\prime}\right) \cup \gamma^{\prime}\right)$ is connected.

Once we have such a handle structure, we then perform a sutured manifold decomposition.

Proposition 6.4. Let $\mathcal{H}(M)$ be a handle structure of a taut sutured manifold $(M, \gamma)$. Suppose that each 0-handle of $\mathcal{F}(M)$ has positive index, and that, for each 0-handle $H_{0}$ of $\mathcal{H}(M), H_{0} \cap(\mathcal{F}(M) \cup \gamma)$ is connected. Let $(M, \gamma) \stackrel{S}{\longrightarrow}\left(M_{S}, \gamma_{S}\right)$ be a taut sutured manifold decomposition, where $\partial S$ has essential intersection with $\mathcal{R}_{ \pm}(M)$ and $[S, \partial S] \neq 0 \in H_{2}(M, \partial M)$. Then there is a surface $S^{\prime}$ properly embedded in $(M, \gamma)$ and a commutative diagram of sutured manifold decompositions and pull-backs

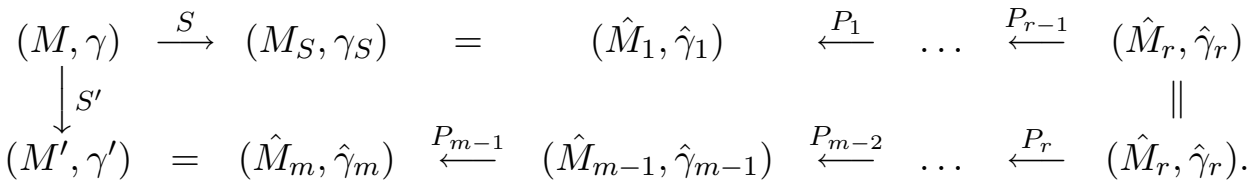

Each $P_{i}$ is either a product disc, an incompressible product annulus or (for $i<r)$ a surface parallel to a subsurface $F_{i}$ of $\mathcal{R}_{ \pm}\left(\hat{M}_{i+1}\right)$, with the orientations of $P_{i}$ and $F_{i}$ disagreeing near $\partial P_{i}$. The induced handle structure $\mathcal{H}\left(M^{\prime}\right)$ on $\left(M^{\prime}, \gamma^{\prime}\right)$ satisfies properties (i), (ii), (iii) and (iv) of Proposition 6.3 and also the following:

(v) For some 0-handle $H_{0}$ of $\mathcal{H}(M), C\left(\mathcal{H}\left(M^{\prime}\right) \cap H_{0}\right)<C\left(H_{0}\right)$.

Proof of Theorems 1.4, 1.5 and 1.6 using Propositions 6.3 and 6.4. Let $\mathcal{H}$ be the dual handle structure for $M$, arising from the generalised triangulation of $M$. We give $M$ the trivial sutured manifold structure with $\mathcal{R}_{-}=\partial M$ and $\mathcal{R}_{+}=\emptyset$. If this is not taut, then $M$ is either reducible or a solid torus. Hence, by Theorem 5.1 of [10], there are no exceptional or norm-exceptional surgery curves in $M$ satisfying the hypotheses of Theorem 1.4. Hence we may assume that $(M, \emptyset)$ is taut.

We will construct a sequence of taut sutured manifolds $\left(M_{i}, \gamma_{i}\right)$ where $1 \leq i \leq n$. The first sutured manifold $\left(M_{1}, \gamma_{1}\right)$ will be $(M, \emptyset)$. Each sutured manifold $\left(M_{i}, \gamma_{i}\right)$ will have a handle structure $\mathcal{H}_{i}$, and there will be an embedding of $M_{i}$ in $M_{i-1}$ having the following properties (some of which are only relevant for $i>1$ ):

(i) $\mathcal{H}_{i}$ respects $\mathcal{H}_{i-1}$.

(ii) For each 0 -handle $H_{0}$ of $\mathcal{H}_{i-1}$, the complexity of $H_{0} \cap \mathcal{H}_{i}$ is no more than that of $H_{0}$. 
(iii) For each 0 -handle $H_{0}$ of $\mathcal{H}_{i-1}$, the intersections

$$
\begin{aligned}
& H_{0} \cap \mathcal{I} H_{i}^{0} \\
& H_{0} \cap \mathcal{I} H_{i}^{0} \cap \mathcal{F}\left(\mathcal{H}_{i}\right) \\
& H_{0} \cap \mathcal{I} H_{i}^{0} \cap \gamma_{i}
\end{aligned}
$$

are each one of a finite number of possibilities (up to trivial modification), which depend only on $\mathcal{F}\left(\mathcal{H}_{i-1}\right) \cap H_{0}$ and $H_{0} \cap \gamma_{i-1}$.

(iv) For a 0 -handle $H_{0}$ of $\mathcal{H}_{i-1}$, if the complexity of $H_{0} \cap \mathcal{H}_{i}$ is equal to that of $H_{0}$, then $H_{0} \cap \mathcal{H}_{i}$ is obtained from $H_{0}$ by a trivial modification.

(v) For some 0-handle $H_{0}$ of $\mathcal{H}_{i-1}(i>2)$, we have $C\left(H_{0} \cap \mathcal{H}_{i}\right)<C\left(H_{0}\right)$.

(vi) For $1<i<n$, each 0-handle $H_{0}$ of $\mathcal{F}\left(\mathcal{H}_{i}\right)$ has positive index, and $H_{0} \cap\left(\mathcal{F}\left(\mathcal{H}_{i}\right) \cup \gamma_{i}\right)$ is connected.

(vii) $K$ lies in $M_{i}$.

(viii) For $1 \leq i<n, H_{2}\left(M_{i}-\operatorname{int}(\mathcal{N}(K)), \partial M_{i}\right) \neq 0$.

(ix) If $M_{i}(\sigma)$ is the manifold obtained from $M_{i}$ by Dehn surgery along $K$ with slope $\sigma$, then at least one of the following is true:

- $M_{i}=M$,

- $\left(M_{i}(\sigma), \gamma_{i}\right)$ is not taut, or

- the core of the surgery solid torus has finite order in $\pi_{1}\left(M_{i}(\sigma)\right)$.

The final manifold $M_{n}$ of the sequence is a solid torus neighbourhood of $K$, plus perhaps some 3-balls. The sequence is constructed using Propositions 6.3 and 6.4 in an alternating fashion.

We now show how to continue this sequence beyond $\left(M_{1}, \gamma_{1}\right)$. We would like to let $(M, \emptyset)=\left(M_{2}, \gamma_{2}\right)$, but (vi) above need not be satisfied in this case. Note, however, that each 0 -handle of $\mathcal{F}(M)$ does indeed have positive index in either of the following cases:

- $M$ is closed (and so we have a genuine triangulation), or

- $\partial M \neq \emptyset$ and we have an ideal triangulation.

In the case where $K$ and $\sigma$ are norm-exceptional, we would like to ensure that one of the above is true. In Theorem 1.6, we explicitly make this assumption. In Theorem 1.4, we alter the given generalised triangulation of $M$ so that it is either genuine or ideal. This can be done algorithmically. Hence, in the case where $K$ and $\sigma$ are norm-exceptional, we let $(M, \emptyset)=\left(M_{2}, \gamma_{2}\right)$.

Suppose now that $K$ and $\sigma$ are exceptional and that some 0 -handle of $\mathcal{F}$ has nonpositive index. Then we use Proposition 6.3 to decompose $(M, \emptyset)$ along product discs and incompressible annuli disjoint from the sutures, resulting in a taut sutured manifold $\left(M^{\prime}, \gamma^{\prime}\right)$ satisfying (i)-(vi) of 6.3 . Since $\Delta(\sigma, \mu)>1$, Theorem 3.2 gives that we may ambient isotope $K$ off each decomposing surface, and hence $K$ lies in $M^{\prime}$.

To apply Proposition 6.3, we need to check that $M$ is not a Seifert fibre space, with base space a disc, having two exceptional fibres, and having $\gamma=\emptyset$. We will suppose it is, and then achieve a contradiction. Let $\alpha$ be 
an arc properly embedded in the base space, separating the two exceptional points. Then $\alpha$ lifts to an annulus $A$ in $M$. Using Theorem 3.2, we may ambient isotope $K$ off $A$. Let $\hat{M}$ be the solid torus which is the closure of the component of $M-A$ containing $K$. Then, $\partial \hat{M}$ is an incompressible torus in $M-\operatorname{int}(\mathcal{N}(K))$. Since $M-\operatorname{int}(\mathcal{N}(K))$ is atoroidal, we deduce that $\partial \hat{M}$ must be parallel to $\partial \mathcal{N}(K)$ and so $K$ is isotopic to the exceptional fibre lying in $\hat{M}$. But then $H_{2}(M-\operatorname{int}(\mathcal{N}(K)), \partial M)$ is trivial, contrary to assumption. Hence, we may apply Proposition 6.3 , and then we let $\left(M_{2}, \gamma_{2}\right)=\left(M^{\prime}, \gamma^{\prime}\right)$.

We now verify (ix). We only applied Proposition 6.3 in the case where $K$ and $\sigma$ are exceptional. Hence, either $\left(M_{K}(\sigma), \emptyset\right)$ is not taut or the core of the surgery solid torus has finite order in $\pi_{1}\left(M_{K}(\sigma)\right)$. We shall show that either $\left(M_{K}^{\prime}(\sigma), \gamma^{\prime}\right)$ is not taut or the core of the surgery solid torus has finite order in $\pi_{1}\left(M_{K}^{\prime}(\sigma)\right)$. Suppose that $\left(M_{K}^{\prime}(\sigma), \gamma^{\prime}\right)$ is taut. Then, by 3.1, the sequence of decompositions

$$
\left(M_{K}(\sigma), \gamma\right) \stackrel{P_{1}}{\longrightarrow} \cdots \stackrel{P_{m}}{\longrightarrow}\left(M_{K}^{\prime}(\sigma), \gamma^{\prime}\right),
$$

is taut. In particular, $\left(M_{K}(\sigma), \gamma\right)$ is taut, and therefore (iii) of 1.3 holds. Also, since each $P_{i}$ has essential intersection with $\mathcal{R}_{ \pm}$, it is therefore incompressible. Therefore, the map $\pi_{1}\left(M_{K}^{\prime}(\sigma)\right) \rightarrow \pi_{1}\left(M_{K}(\sigma)\right)$ induced by inclusion is an injection. Therefore, the core of the surgery solid torus has finite order in $\pi_{1}\left(M_{K}^{\prime}(\sigma)\right)$.

Thus, we have now constructed $\left(M_{2}, \gamma_{2}\right)$ and have verified that it has the correct properties. Suppose that we have constructed a sequence as far as $\left(M_{i}, \gamma_{i}\right)$, satisfying (i)-(ix) above. If $H_{2}\left(M_{i}-\operatorname{int}(\mathcal{N}(K)), \partial M_{i}\right)$ is trivial, then we stop. If this homology group is nontrivial, then (see Section 3 or Theorem 2.6 of [10]) we may find a taut decomposition

$$
\left(M_{i}-\operatorname{int}(\mathcal{N}(K)), \gamma_{i}\right) \stackrel{S}{\longrightarrow}\left(M_{i}^{\prime}-\operatorname{int}(\mathcal{N}(K)), \gamma_{i}^{\prime}\right),
$$

such that

- $S$ is disjoint from $\partial \mathcal{N}(K)$,

- no curve of $\partial S$ bounds a disc in $\mathcal{R}_{ \pm}\left(M_{i}\right)$,

- no component $X$ of $M_{i}^{\prime}$ has $\partial X \subset \mathcal{R}_{-}\left(M_{i}^{\prime}\right)$ or $\partial X \subset \mathcal{R}_{+}\left(M_{i}^{\prime}\right)$, and

- $[S, \partial S] \neq 0 \in H_{2}\left(M_{i}-\operatorname{int}(\mathcal{N}(K)), \partial M_{i}\right)$.

This implies that $[S, \partial S] \neq 0 \in H_{2}\left(M_{i}, \partial M_{i}\right)$. In the case where $K$ and $\sigma$ are norm-exceptional and $M_{i}=M$, we insist that $[S, \partial S]=z \in H_{2}(M-$ $\operatorname{int}(\mathcal{N}(K)), \partial M)$, where $z$ is the homology class in Definition 1.3.

Let $M_{i}^{\prime}(\sigma)$ be the result of $M_{i}^{\prime}$ after Dehn surgery along $K$ with slope $\sigma$. Since $\left(M_{i}(\sigma), \gamma_{i}\right)$ is not taut or the core of the surgery solid torus has finite order in $\pi_{1}\left(M_{i}(\sigma)\right)$, the argument above gives that $\left(M_{i}^{\prime}(\sigma), \gamma_{i}\right)$ is not taut or the core of the surgery solid torus has finite order in $\pi_{1}\left(M_{i}^{\prime}(\sigma)\right)$. Using the argument in Section 3 (see also Theorem 1.8 of [1]), we deduce that the decomposition $\left(M_{i}, \gamma_{i}\right) \stackrel{S}{\longrightarrow}\left(M_{i}^{\prime}, \gamma_{i}^{\prime}\right)$ is taut. Since each 0-handle of $\mathcal{F}\left(\mathcal{H}_{i}\right)$ 
has positive index, we may apply Proposition 6.4 to $\left(M_{i}, \gamma_{i}\right)$, and so end with a sutured manifold $\left(M_{i}^{\prime \prime}, \gamma_{i}^{\prime \prime}\right)$ with a handle-decomposition $\mathcal{H}_{i}^{\prime \prime}$, satisfying (i)(iv) of 6.3 and (v) of 6.4. Again using 3.2, we may isotope $K$ off each product disc and incompressible product annulus, and so we may assume $K$ lies in $M_{i}^{\prime \prime}$. Also, using the commutative diagram in Proposition 6.4, we deduce that $\left(M_{i}^{\prime \prime}(\sigma), \gamma_{i}^{\prime \prime}\right)$ is not taut or the core of the surgery solid torus has finite order in $\pi_{1}\left(M_{i}^{\prime \prime}(\sigma)\right)$.

If any component of $M_{i}^{\prime \prime}$ is a solid torus disjoint from $K$, we decompose it along a meridian disc. If the component $X$ of $M_{i}^{\prime \prime}$ containing $K$ is a solid torus, then the atoroidality of $M-\operatorname{int}(\mathcal{N}(K))$ implies that $K$ is the core of $X$. In this case, we set $\left(M_{n}, \gamma_{n}\right)=\left(X, \gamma_{i}^{\prime \prime} \cap X\right)$, together with some 3-balls obtained by decomposing $M_{i}^{\prime \prime}-X$.

We may therefore assume that no component of $M_{i}^{\prime \prime}$ is a solid torus, and so we can apply 6.3 to $\left(M_{i}^{\prime \prime}, \gamma_{i}^{\prime \prime}\right)$ to obtain a sutured manifold $\left(M_{i+1}, \gamma_{i+1}\right)$ satisfying (i)-(ix) above. Note that each component of $\mathcal{F}\left(\mathcal{H}_{i+1}\right)$ has positive index, and therefore, the only 0 -handles of $\mathcal{H}_{i+1}$ not lying in $\mathcal{I} H_{i+1}^{0}$ are handles disjoint from $\mathcal{F}\left(\mathcal{H}_{i+1}\right)$.

By (ii), (v) and Lemma 5.4, the complexity of $\mathcal{H}_{i+1}$ is strictly less than that of $\mathcal{H}_{i}$. Hence, eventually, the sequence terminates with a sutured manifold $\left(M_{n}, \gamma_{n}\right)$ such that $H_{2}\left(M_{n}-\operatorname{int}(\mathcal{N}(K)), \partial M_{n}\right)=0$. Then $M_{n}$ is a solid torus neighbourhood of $K$, plus perhaps some 3 -balls. By (ix), the sutures $\gamma_{n} \cap \mathcal{N}(K)$ are parallel to $\sigma$.

Note that, for each 0 -handle $H_{0}$ of $\mathcal{H}(M)$, there are only finitely many possibilities for $\mathcal{F}(M) \cap H_{0}$. Thus, by induction on complexity using (ii), (iii) and (iv) above, there is in each 0-handle $H_{0}$ of $\mathcal{H}(M)$, only a finite number of possibilities for

$$
\begin{aligned}
& H_{0} \cap \mathcal{I} H_{n}^{0} \\
& H_{0} \cap \mathcal{I} H_{n}^{0} \cap \mathcal{F}\left(\mathcal{H}_{n}\right) \\
& H_{0} \cap \mathcal{I} H_{n}^{0} \cap \gamma_{n} .
\end{aligned}
$$

Each possibility for $H_{0} \cap \mathcal{I} H_{n}^{0} \cap \gamma_{n}$ gives a tangle in the associated 3-simplex of $M$. These tangles join to form $\gamma_{n}$ (with possibly some unknotted curves removed). Some component of $\gamma_{n}$ is the $\sigma$-cable of $K$, and hence the tangles required for Theorems 1.5 and 1.6 are constructed by taking all possible subtangles of $H_{0} \cap \mathcal{I} H_{n}^{0} \cap \gamma_{n}$.

Each possibility for $H_{0} \cap \mathcal{I} H_{n}^{0}$ and $H_{0} \cap \mathcal{I} H_{n}^{0} \cap \mathcal{F}^{0}\left(\mathcal{H}_{n}\right)$ gives a graph $G$ in the associated 3-simplex of $M$. When the collection of these graphs (one in each 3-simplex of $M$ ) are joined, they form the 0-handles and 1-handles of $M_{n}$. The 2-handles of $M_{n}$ are attached along the annuli $\left(\mathcal{H}_{n}^{0} \cup \mathcal{H}_{n}^{1}\right) \cap$ $\mathcal{H}_{n}^{2}$, which are determined by $\mathcal{H}_{n}^{0} \cap \mathcal{F}^{1}\left(\mathcal{H}_{n}\right)$. Thus, we readily see that the algorithm given in Section 2 constructs all possibilities for $K$ and $\sigma$. Hence, Theorem 1.4 is established. 
We end this section with the following:

Proof of Theorem 1.1.

This is an almost immediate corollary of Theorem 1.4, but there is one complication. If we set $M=S^{3}-\operatorname{int}(\mathcal{N}(L))$, then it is not obvious that $M-\operatorname{int}(\mathcal{N}(K))$ is atoroidal. To establish this, we will use a modified form of the argument in Proposition 2.3 of [6].

Suppose therefore that $T$ is an incompressible torus in $M-\operatorname{int}(\mathcal{N}(K))$ which is not parallel to $\partial \mathcal{N}(K)$ or $\partial \mathcal{N}(L)$. Since we are assuming that $L$ is not a nontrivial satellite knot, then $T$ must be compressible in $M$ or be parallel to $\partial \mathcal{N}(L)$. In the latter case, $K$ lies in the collar between $T$ and $\partial \mathcal{N}(L)$, and then it is easy to see that $L^{\prime}$ is a winding number one satellite of $L$. In particular, genus $\left(L^{\prime}\right) \geq \operatorname{genus}(L)$, which is contrary to hypothesis. Therefore, $T$ must be compressible in $M$. There are now two cases.

Case 1. $T$ lies in a 3-ball in $M$.

Then, $T$ separates $S^{3}$ into a nontrivial knot exterior $X$ disjoint from $K$ and $L$, and a solid torus containing $K$ and $L$. Let $Y$ be the manifold obtained from $M$ by removing the interior of $X$. Let $Y^{\prime}$ be the manifold obtained from $S^{3}-\operatorname{int}\left(\mathcal{N}\left(L^{\prime}\right)\right)$ by removing the corresponding knot exterior, which we also call $X$. Note that $H_{2}(Y-\operatorname{int}(\mathcal{N}(K)), \partial Y)$ is nontrivial. Also, $\partial Y$ has compressible boundary. So, by Theorem 5.1 of [10] (see also the argument in Section 3) the minimal genus of a Seifert surface for $L^{\prime}$ in the complement of $X$ is at least the genus of $L$ in the complement of $X$, which is the genus of $L$. Since $X$ is a nontrivial knot exterior, the minimal genus of a Seifert surface for $L^{\prime}$ in the complement of $X$ is just the genus of $L^{\prime}$. So, in this case, the genus of $L^{\prime}$ is at least the genus of $L$, which is a contradiction.

Case 2. $T$ bounds a solid torus $V$ in $M$ which contains $K$.

Let $V^{\prime}$ be the manifold obtained from $V$ by $1 / q$ Dehn surgery along $K$.

Case $2 \mathrm{~A} . K$ has winding number zero in $V$.

Then consider a minimal genus Seifert surface $S$ for the knot $L^{\prime}$. We may assume that it intersects $\partial V^{\prime}$ in a collection of simple closed curves, which inherit an orientation from $S$. The union of these curves is homologically trivial in $V^{\prime}$. Hence, by making annular modifications to $S$, if necessary, which do not increase its genus, we may assume that each curve of $S \cap \partial V^{\prime}$ is homologically trivial in $V^{\prime}$. Since $K$ has winding number zero in $V$, these curves are also homologically trivial in $V$. Hence, we may fill them in with meridian discs in $V$. This gives a Seifert surface for $L$ with genus at most that of $S$, which is a contradiction.

Case 2B. $K$ has nonzero winding number in $V$.

Since $K$ and $L$ have zero linking number, so do $L$ and the core of $V$. Therefore, there exists a Seifert surface $S^{\prime}$ for $L^{\prime}$ which is disjoint from $V^{\prime}$. 
If $\partial V^{\prime}$ is incompressible in $V^{\prime}$, then by Lemma A.16 of [7], there is a minimal genus Seifert surface for $L^{\prime}$ which is disjoint from $V^{\prime}$. This gives a Seifert surface for $L$, and again we reach the contradiction that genus $(L) \leq$ $\operatorname{genus}\left(L^{\prime}\right)$.

So, we may assume that $\partial V^{\prime}$ is compressible in $V^{\prime}$. This implies that $V^{\prime}$ is a solid torus, since it cannot be reducible, as it lies in $S^{3}$. Now, $1 / q$ surgery along a knot $K$ in the solid torus $V$ never yields another solid torus if $|q|>1$, unless $K$ is a core of $V$ or $K$ lies in a 3-ball in $V$ [9]. If $K$ lies in a 3-ball in $V$, then $1 / q$ surgery along $K$ does not alter $L$, which is a contradiction. If $K$ is a core of $V$, then $T$ is parallel to $\partial \mathcal{N}(K)$, contradicting the assumption that it is essential.

This proves then that $M-\operatorname{int}(\mathcal{N}(K))$ is atoroidal. Theorem 1.1 now follows directly from Theorem 1.4.

\section{Simplifying handle structures.}

In the next two sections, we will give a proof of Proposition 6.3. In particular, we will assume that each component of $M$ has nonempty boundary, and that no component of $M$ is a solid torus or a Seifert fibre space as in 6.3. We start by giving various elementary procedures for simplifying a handle structure $\mathcal{H}$ of the taut sutured manifold $(M, \gamma)$. Our aim is to end with a handle structure in which each 0 -handle of $\mathcal{F}$ has positive index. Each procedure will satisfy (i)-(iv) of 6.3. It will not increase the complexity of the handle structure, but it need not decrease it. To ensure that these procedures eventually terminate, we therefore introduce the following definition:

Definition 7.1. Let $\mathcal{H}$ be a handle structure for a sutured manifold $(M, \gamma)$. Define the extended $\mathcal{F}$-complexity for $\mathcal{H}$ to be the set of triples

$$
C_{\mathcal{F}}^{+}(\mathcal{H})=\left\{\left(C_{1}(F), C_{2}(F), C_{3}(F)\right): F \text { a component of } \mathcal{F}\right\},
$$

where repetitions are retained. Here, $C_{1}, C_{2}$ and $C_{3}$ are the integers defined in Section 5. We also define the extended complexity $C^{+}(\mathcal{H})$ to be the ordered pair $\left(C_{\mathcal{F}}^{+}(\mathcal{H}), n(\mathcal{H})\right)$.

The difference between the extended $\mathcal{F}$-complexity and the $\mathcal{F}$-complexity of a handle structure is that extended $\mathcal{F}$-complexity also takes into account components of $\mathcal{F}$ with nonpositive index.

We order the extended $\mathcal{F}$-complexities and extended complexities as we do the $\mathcal{F}$-complexities and complexities of handle structures (see Section 5). As in Lemma 5.3, this is a well-ordering. The procedures we give in the next two sections will all reduce the extended complexity of the handle structure, and so are guaranteed to terminate.

The following lemma will be useful in our verification that (i)-(iv) of 6.3 holds and that extended complexity is reduced: 
Lemma 7.2. Let $(M, \gamma)$ be a sutured manifold with a handle structure $\mathcal{H}$. Let $\left(M^{\prime}, \gamma^{\prime}\right)$ be embedded in $M$, with a handle structure $\mathcal{H}^{\prime}$ which respects $\mathcal{H}$. Suppose that, for each component $F$ of $\mathcal{F}$, either $C_{\mathcal{F}}^{+}\left(F \cap \mathcal{F}^{\prime}\right)<C_{\mathcal{F}}^{+}(F)$ or $F \subset \mathcal{F}^{\prime}$. Suppose also that the former of the above two possibilities holds for at least one component $F$ of $\mathcal{F}$. Suppose also that, if $I(F) \leq 0$, then the index of each component of $F \cap \mathcal{F}^{\prime}$ is nonpositive. Then, $C_{\mathcal{F}}^{+}\left(\mathcal{H}^{\prime}\right)<C_{\mathcal{F}}^{+}(\mathcal{H})$, and (i), (ii) and (iv) of 6.3 are verified.

Proof. A version of the argument in Lemma 5.4 gives that $C_{\mathcal{F}}^{+}\left(\mathcal{H}^{\prime}\right)<C_{\mathcal{F}}^{+}(\mathcal{H})$. We now check (ii) and (iv) of 6.3. (Note that (i) of 6.3 is part of the hypothesis of the lemma.) For each component $F$ of $\mathcal{F}$, we have one of the following possibilities:

(i) $F \subset \mathcal{F}^{\prime}$ and so $F \cap \mathcal{F}^{\prime}$ is a copy of $F$, or

(ii) $I(F)>0$ and $C_{\mathcal{F}}\left(F \cap \mathcal{F}^{\prime}\right) \leq C_{\mathcal{F}}^{+}\left(F \cap \mathcal{F}^{\prime}\right)<C_{\mathcal{F}}^{+}(F)=C_{\mathcal{F}}(F)$, or

(iii) $I(F) \leq 0$ and $C_{\mathcal{F}}\left(F \cap \mathcal{F}^{\prime}\right)=C_{\mathcal{F}}(F)$.

In (iii), we are using that if $I(F) \leq 0$, then the index of each component of $F \cap \mathcal{F}^{\prime}$ is nonpositive, and so does not contribute to $\mathcal{F}$-complexity. Therefore, for any 0-handle $H_{0}$ of $\mathcal{H}, C_{\mathcal{F}}\left(\mathcal{H}^{\prime} \cap H_{0}\right) \leq C_{\mathcal{F}}\left(H_{0}\right)$. Also, if we have equality, then (ii) above cannot occur for any component $F$ of $\mathcal{F} \cap H_{0}$, which implies that components $F$ of $\mathcal{F} \cap H_{0}$ with positive index remain unchanged and hence that $n\left(\mathcal{H}^{\prime} \cap H_{0}\right) \geq n\left(H_{0}\right)$. So, $C\left(\mathcal{H}^{\prime} \cap H_{0}\right) \leq C\left(H_{0}\right)$. This verifies (ii) of 6.3. Also, if $C\left(\mathcal{H}^{\prime} \cap H_{0}\right)=C\left(H_{0}\right)$, then $\mathcal{H}^{\prime} \cap H_{0}$ is obtained from $H_{0}$ by a trivial modification, verifying (iv) of 6.3 .

Before we describe the procedures in detail, we mention that many of them simply remove some handles of $\mathcal{H}$. The following lemma will therefore be useful:

Lemma 7.3. Let $\mathcal{H}^{\prime}$ be a collection of handles of $\mathcal{H}$ forming a 3-manifold $M^{\prime}$ embedded in $M$, with $\mathcal{H}-\mathcal{H}^{\prime}$ containing at least one $i$-handle for some $i \leq 2$. Suppose that $\mathcal{H}^{\prime}$ is a handle structure, that each handle of $\mathcal{H}-\mathcal{H}^{\prime}$ is disjoint from $\gamma$ and that $\left(M^{\prime}, \gamma\right)$ is a sutured manifold structure. Then, (i)-(iv) of 6.3 are satisfied, and extended $\mathcal{F}$-complexity is reduced.

Proof. It follows straight from the definition that $\mathcal{H}^{\prime}$ respects $\mathcal{H}$. Let us now check that the hypotheses of 7.2 hold. Let $F$ be some component of $\mathcal{F}$ and let $F^{\prime}=F \cap \mathcal{F}^{\prime}$. The 1-handles of $F$ are either removed or divided up amongst the components of $F^{\prime}$. In particular, each component $X$ of $F^{\prime}$ has $C_{1}(X) \leq C_{1}(F)$. If this inequality is an equality for some $X$, then in fact $F^{\prime}=F$. Hence, either $C_{\mathcal{F}}^{+}\left(F^{\prime}\right)<C_{\mathcal{F}}^{+}(F)$ or $F \subset \mathcal{F}^{\prime}$. Also, the former case holds for some component $F$ of $\mathcal{F}$.

We now check that if $I(F) \leq 0$, then each component of $F^{\prime}$ has nonpositive index. But $F^{\prime}$ is obtained from $F$ by removing some 1-handles (or equivalently, cutting $F$ along properly embedded arcs), then removing some 0 -handles disjoint from $\gamma$. Thus, each component $X$ of $F^{\prime}$ has $\chi(X) \geq \chi(F)$ 
and $\gamma \cap X \leq \gamma \cap F$. Hence, $X$ does not have positive index if $F$ does not have positive index.

Thus, by Lemma 7.2, (i), (ii) and (iv) of 6.3 hold and extended $\mathcal{F}$ complexity is reduced. Also, (iii) of 6.3 is obvious.

Procedure 1. Slicing a 0-handle along a disc.

Suppose that there is a disc $D$ properly embedded in some 0 -handle $H_{0}$ with $D \cap \gamma=D \cap \mathcal{F}=\emptyset$, and which separates $\mathcal{F} \cap H_{0}$. Then, $\partial D$ either lies in $\partial \mathcal{H}^{3}$ or in $\mathcal{R}_{ \pm}$. In the former case, $\partial D$ bounds a disc $D^{\prime}$ in $\partial \mathcal{H}^{3}$, since each component of $\partial \mathcal{H}^{3}$ is a sphere. In the case where $\partial D$ lies in $\mathcal{R}_{ \pm}$, the incompressibility of $\mathcal{R}_{ \pm}$implies that $\partial D$ bounds a disc $D^{\prime}$ in $\mathcal{R}_{ \pm}$. The irreducibility of $M$ implies that $D \cup D^{\prime}$ bounds a ball $B$ in $M$. Procedure 1 is the removal of all handles of $\mathcal{H}$ which intersect int $(B)$, other than $H_{0}$. If $D^{\prime} \subset$ $\partial \mathcal{H}^{3}$, we extend $\mathcal{H}^{3}$ over $B$. By Lemma 7.3 , (i)-(iv) of 6.3 hold, and extended $\mathcal{F}$-complexity is reduced. Thus, using this procedure, we eventually obtain a handle structure $\mathcal{H}\left(M^{\prime}\right)$ on the resulting sutured manifold $\left(M^{\prime}, \gamma^{\prime}\right)$, with $H_{0}^{\prime} \cap\left(\mathcal{F}\left(M^{\prime}\right) \cup \gamma^{\prime}\right)$ connected, for each 0-handle $H_{0}^{\prime}$ of $\mathcal{H}\left(M^{\prime}\right)$. This is (vi) of 6.3 .

Procedure 2. Collapsing a 2-handle and a 1-handle disjoint from $\gamma$.

Suppose now that $H_{1}$ is a 1-handle of $M$ which is disjoint from $\gamma$ and which intersects $\mathcal{H}^{2}$ in a single disc. Then this disc is contained in a single 2-handle $H_{2}$. We may remove $H_{1}$ and $H_{2}$, without changing the homeomorphism type of $(M, \gamma)$. Lemma 7.3 gives that (i)-(iv) of 6.3 are satisfied, and that extended $\mathcal{F}$-complexity is reduced.

Procedure 3. Collapsing a 2-handle and a 1-handle containing an arc of $\gamma$.

Let $H_{1}$ be a 1-handle of $M$ which intersects $\gamma$ in a single arc, and which intersects $\mathcal{H}^{2}$ in a single disc, lying in a 2-handle $H_{2}$. Procedure 3 is the collapsing of $H_{1}$ and $H_{2}$. This moves $\gamma \cap H_{1}$ onto an arc running along $\partial\left(\mathcal{H}^{0} \cup \mathcal{H}^{1}-H_{1}\right)$. Let $\left(M^{\prime}, \gamma^{\prime}\right)$ be the new sutured manifold, with handle structure $\mathcal{H}^{\prime}$.

This procedure has the following effect on $\mathcal{F}$ : Removing $H_{2} \cap \partial \mathcal{H}^{0}$ (which is a collection of 1 -handles of $\mathcal{F}$ ), removing $H_{1} \cap \partial \mathcal{H}^{0}$ (which is precisely two 0 -handles of $\mathcal{F}$ ) and then replacing each handle of $\mathcal{F}$ which we have removed with a sub-arc of $\gamma$. Thus, if $F$ is a component of $\mathcal{F}$, and $F^{\prime}=F \cap \mathcal{F}^{\prime}$, then each component of $X$ of $F^{\prime}$ has $C_{1}(X) \leq C_{1}(F)$, and if we have equality for some component $X$, then in fact $F$ is unchanged by the procedure. This verifies one of the hypotheses of Lemma 7.2.

We now check that if $F$ has nonpositive index, then each component $X$ of $F^{\prime}$ has nonpositive index. Suppose therefore $F$ has nonpositive index and that $F$ is changed by the procedure. It is simple to show that $I(F)$ is the 
sum of the indices of $X$, as $X$ ranges over all components of $F^{\prime}$. Therefore, the only way that a component $X$ of $F^{\prime}$ can have positive index is if another component of $F^{\prime}$ has negative index. However, since $F$ is changed by the procedure, then each component of $F^{\prime}$ touches $\gamma^{\prime}$, and hence $I(X) \geq 0$ for all components $X$ of $F^{\prime}$. Lemma 7.2 now gives us that extended $\mathcal{F}$-complexity decreases and that (i), (ii) and (iv) of 6.3 hold. It is straightforward to verify (iii) of 6.3 .

Procedure 4. Decomposing along a product disc, then sliding $\gamma$.

Suppose that $H_{1}$ is a 1-handle of $\mathcal{H}$ which is disjoint from $\mathcal{H}^{2}$ and which has $\left|H_{1} \cap \gamma\right|=2$. Let $D$ be one of the two discs of $H_{1} \cap \mathcal{H}^{0}$, lying in some 0 -handle $H_{0}$. Push $D$ a little into $H_{0}$. Then $D$ is a product disc, which we decompose along. This decomposition creates a new handle decomposition which respects $\mathcal{H}$, and leaves both the complexity and extended complexity of $\mathcal{H}$ unchanged. But now the two arcs of $H_{1} \cap \gamma$ are joined by an arc of $\mathcal{H}^{0} \cap \gamma$. We may therefore perform an ambient isotopy which slides $\gamma$ off $H_{1}$. Then, using Procedure 1 , we may remove $H_{1}$. Again, (i)-(iv) of 6.3 are satisfied and extended $\mathcal{F}$-complexity is reduced.

Procedure 5. Collapsing a 3-ball disjoint from $\gamma$.

Suppose that a component of $M$ is a 3-ball disjoint from $\gamma$, comprised of two 0-handles joined by a 1-handle. Then we may collapse the 1-handle and one of the 0 -handles. This reduces the extended $\mathcal{F}$-complexity and (i)-(iv) of 6.3 are satisfied.

Procedure 6. Collapsing a 2-handle and a 3-handle.

Let $H_{2}=D^{1} \times D^{2}$ be a 2-handle, with one component of $\partial D^{1} \times D^{2}$ in $\partial M$, and the other component touching a 3-handle $H_{3}$. Then we may remove $H_{2}$ and $H_{3}$ without changing the homeomorphism type of $M$. By Lemma 7.3, this procedure reduces extended $\mathcal{F}$-complexity and satisfies (i)-(iv) of 6.3 . Note that we are assuming in 6.3 that each component of $M$ has nonempty boundary. Hence, if $\mathcal{H}^{3}$ is nonempty, we may always apply this procedure somewhere. In this way, we remove all 3 -handles from $M$.

The above six procedures are not enough to ensure that each 0-handle of $\mathcal{F}$ has positive index. To deal with components of $\mathcal{F}$ which are annuli disjoint from $\gamma$, we must clump collections of handles into groups, known as amalgams, which are defined as follows:

Definition 7.4. An amalgam $\mathcal{A}$ is a connected collection of handles with the following properties:

(i) $\mathcal{A}$ is disjoint from $\gamma$,

(ii) $\mathcal{A}$ is an $I$-bundle over a connected surface $G$,

(iii) the $(I-\partial I)$-bundle over $\partial G$ is disjoint from $\partial M \cup \partial \mathcal{H}^{3}$, 
(iv) the handles of $\mathcal{A}$ touching the $(I-\partial I)$-bundle over $\partial G$ are 1-handles and 2-handles,

(v) no 2-handle or 3 -handle of $\mathcal{H}-\mathcal{A}$ touches $\mathcal{A}$,

(vi) the $\partial I$-bundle over $G$ lies in $\mathcal{R}_{ \pm}$, and

(vii) $\operatorname{cl}(\mathcal{H}-\mathcal{A})$ inherits a handle structure from $\mathcal{H}$.

An amalgam is trivial if it is a single 2-handle; otherwise it is nontrivial.

An amalgam $\mathcal{A}$ behaves in many ways just like a 2 -handle. For example, it is attached onto the 0 -handles and 1-handles of $\mathcal{H}-\mathcal{A}$ in a fashion that is very similar to the attachment of a 2 -handle.

The main example of a nontrivial amalgam $\mathcal{A}$ is a connected collection of 2-handles and 1-handles disjoint from $\gamma$ and $\mathcal{H}^{3}$, such that each 1-handle of $\mathcal{A}$ intersects $\mathcal{H}^{2}$ in precisely two discs, and these discs lie in 2-handles of $\mathcal{A}$. For then the co-core $D^{2}$ of each 1-handle $H_{1}=D^{2} \times D^{1}$ in $\mathcal{A}$ has a product structure as $I \times I$, in which $H_{1} \cap \mathcal{H}^{2}=\partial I \times I \times D^{1}$. The product structures on the 1-handles combine with the product structures on the 2-handles to form an $I$-bundle structure on $\mathcal{A}$ with the required properties. An example is given in Figure 7.5.

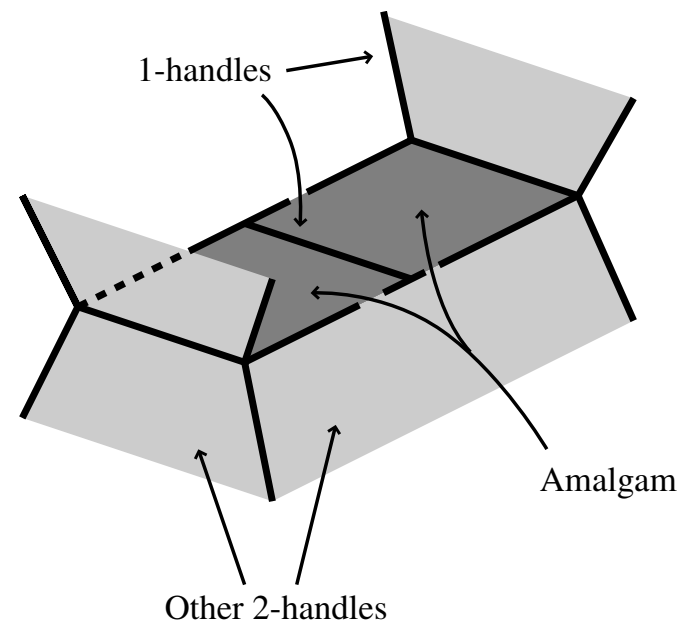

Figure 7.5.

In Section 8, we will show how to remove all nontrivial amalgams. This, together with Procedures 1-6 is enough to ensure that each 0 -handle of $\mathcal{F}$ has positive index.

Lemma 7.6. Let $\mathcal{H}$ be a handle-decomposition of a connected sutured manifold with nonempty boundary, containing no nontrivial amalgams. If some 0 -handle of $\mathcal{F}$ has nonpositive index, then we may apply one of Procedures 16. 
Proof. Let $V$ be a 0 -handle of $\mathcal{F}$ with nonpositive index. Then, there are a number of cases.

1. $\left|\mathcal{H}^{2} \cap V\right|=0$ and $|\gamma \cap V|=0$.

2. $\left|\mathcal{H}^{2} \cap V\right|=1$ and $|\gamma \cap V|=0$.

3. $\left|\mathcal{H}^{2} \cap V\right|=1$ and $|\gamma \cap V|=1$.

4. $\left|\mathcal{H}^{2} \cap V\right|=0$ and $|\gamma \cap V|=2$.

5. $\left|\mathcal{H}^{2} \cap V\right|=0$ and $|\gamma \cap V|=1$.

6. $\left|\mathcal{H}^{2} \cap V\right|=2$ and $|\gamma \cap V|=0$.

For $i=2,3$ and 4, we may apply Procedure $i$. In Case 1 , let $H_{1}$ be the 1-handle containing $V$, and let $V^{\prime}$ be $\partial H_{1} \cap \partial \mathcal{H}^{0}-V$. If we cannot apply Procedure 1 to either of the discs $V$ or $V^{\prime}$, then this component of $M$ is a 3ball disjoint from $\gamma$, comprised of two 0-handles joined by a single 1-handle. We may therefore apply Procedure 5. Case 5 cannot arise since $\gamma$ separates $\partial M$ into $\mathcal{R}_{-}$and $\mathcal{R}_{+}$. By applying Procedure 6 if necessary, we may assume that $M$ contains no 3 -handles. In Case 6 , the 1 -handle of $\mathcal{H}$ containing $V$ is part of a nontrivial amalgam, contrary to assumption.

\section{Removing nontrivial amalgams.}

We now give a procedure for removing all nontrivial amalgams, which will complete the proof of Proposition 6.3. Suppose that there is a nontrivial amalgam $\mathcal{A}$ in the handle structure $\mathcal{H}$ of the taut sutured manifold $(M, \gamma)$. We will assume that $\mathcal{A}$ is maximal, in the sense that if any other handles are added to $\mathcal{A}$, the resulting collection of handles does not form an amalgam. We will also assume that we cannot apply any of Procedures 1-6 in Section 7. In particular, due to Procedure 6, this implies that $M$ has no 3 -handles. Recall that $\mathcal{A}$ has the structure of an $I$-bundle over a connected surface $G$. The $I$-bundle over $\partial G$ will be denoted by $\partial_{v} \mathcal{A}$.

Note that (iii) of 7.4 implies that $\partial_{v} \mathcal{A}$ is a union of intersections between handles of $\mathcal{H}$. By (v) of 7.4, only 0-handles and 1-handles of $\mathcal{H}-\mathcal{A}$ touch $\partial_{v} \mathcal{A}$. By (iv) of 7.4 , only 1 -handles and 2 -handles of $\mathcal{A}$ touch $\partial_{v} \mathcal{A}$. Hence, we may define a handle structure on $\partial_{v} \mathcal{A}$ as follows: The 0-handles of $\partial_{v} \mathcal{A}$ arise from the intersection of 1 -handles of $\mathcal{A}$ with the 0 -handles of $\mathcal{H}-\mathcal{A}$. The 1-handles of $\partial_{v} \mathcal{A}$ arise from the intersection of 2-handles of $\mathcal{A}$ with 0-handles and 1-handles of $\mathcal{H}-\mathcal{A}$.

Lemma 8.1. Suppose that we cannot apply Procedure 2 of Section 7. Then each 0-handle of $\partial_{v} \mathcal{A}$ abuts precisely two 1-handles of $\partial_{v} \mathcal{A}$.

Proof. If not, then some 0-handle of $\partial_{v} \mathcal{A}$ abuts precisely one 1-handle of $\partial_{v} \mathcal{A}$, since $\partial_{v} \mathcal{A}$ is a collection of annuli. This 0 -handle $D$ is a component of $\mathcal{H}^{1} \cap \mathcal{H}^{0}$. Let $H_{0}$ (respectively, $H_{1}$ ) be the 0-handle (respectively, the 1-handle) of $\mathcal{H}$ containing $D$. Then $H_{1}$ lies in $\mathcal{A}$, but $H_{0}$ does not. Since $D$ abuts precisely one 1 -handle of $\partial_{v} \mathcal{A}, H_{1}$ intersects $\mathcal{A} \cap \mathcal{H}^{2}$ in a single disc, 
lying in some 2-handle $H_{2}$. This in fact must the only intersection between $H_{1}$ and $\mathcal{H}^{2}$, by (v) of 7.4. Hence, we may apply Procedure 2 of Section 7 to $H_{1}$ and $H_{2}$, which is a contradiction.

The following lemma will also be useful:

Lemma 8.2. Let $\mathcal{H}$ be a handle structure for $(M, \gamma)$ to which we cannot apply any of Procedures 1-6. Let $\mathcal{A}$ be a maximal amalgam in $\mathcal{H}$. Let $F$ be a component of $\mathcal{F}$ touching $\partial_{v} \mathcal{A}$. Then $F$ has positive index.

Proof. Since we cannot apply any of Procedures 1-6, the only 0-handles of $\mathcal{F}$ with nonpositive index have valence two and are disjoint from $\gamma$ (see the proof of Lemma 7.6). If $F$ has nonpositive index then each 0-handle of $F$ must be of this form. Hence, $F$ is an annulus disjoint from $\gamma$. Therefore, $F$ must be the only component of $\mathcal{F}$ lying in $H_{0}$, where $H_{0}$ is the 0 -handle of $\mathcal{H}$ containing $F$. For, otherwise we could apply Procedure 1 .

Consider a handle of $F$ lying in $\partial_{v} \mathcal{A}$. This is a component of intersection between $H_{0}$ and some 1-handle or 2-handle of $\mathcal{H}$. By (iv) of 7.4, we must have $H_{0} \notin \mathcal{A}$.

If $F$ lies entirely in $\partial_{v} \mathcal{A}$, then each handle of $\mathcal{H}$ touching $H_{0}$ must be in $\mathcal{A}$, and so we may extend $\mathcal{A}$ over $H_{0}$, contradicting its maximality. Therefore, $F \cap \partial_{v} \mathcal{A}$ is not the whole of $F$.

If $V$ is a 0 -handle of $F$ lying in $\partial_{v} \mathcal{A}$, then the 1-handle of $\mathcal{H}$ touching $V$ must lie in $\mathcal{A}$. Hence, by (v) of 7.4, the 1-handles of $F$ touching $V$ also lie in $\partial_{v} \mathcal{A}$. Hence, we may find a 0 -handle $F_{0}$ of $F$ and a 1 -handle $F_{1}$ of $F$ which are adjacent, with $F_{1}$ in $\partial_{v} \mathcal{A}$, but $F_{0}$ not in $\partial_{v} \mathcal{A}$. Let $H_{1}$ (respectively, $H_{2}$ ) be the 1-handle (respectively, 2-handle) of $\mathcal{H}$ containing $F_{0}$ (respectively, $F_{1}$ ). Then, we must have $H_{0} \notin \mathcal{A}, H_{1} \notin \mathcal{A}$ and $H_{2} \in \mathcal{A}$. Let $H_{2}^{\prime}$ be the 2-handle other than $H_{2}$ which touches $H_{1}$. (If $H_{2}$ touches $H_{1}$ in two discs, then let $H_{2}^{\prime}=H_{2}$.) If $H_{2}^{\prime} \in \mathcal{A}$, then we may extend $\mathcal{A}$ over $H_{1}$. If $H_{2}^{\prime} \notin \mathcal{A}$, we may extend $\mathcal{A}$ over $H_{1} \cup H_{2}^{\prime}$. In each case, the maximality of $\mathcal{A}$ is contradicted.

We now consider the various possibilities for $\mathcal{A}$ case by case.

Case 1. $\mathcal{A}$ is an $I$-bundle over a $\operatorname{disc} G$.

In this case, we replace $\mathcal{A}$ with a single 2-handle $H_{2}$. We attach $H_{2}$ to $\mathcal{H}^{0} \cup \mathcal{H}^{1}-\mathcal{A}$ using the annulus $\partial_{v} \mathcal{A}$. We now check that $\mathcal{H}^{\prime}=(\mathcal{H}-\mathcal{A}) \cup H_{2}$ is a handle structure. By (vii) of 7.4, the only requirement that is not immediately obvious is that $H_{2}$ touches some 1-handle. But, if not, then $\partial_{v} \mathcal{A}$ would have been an annular component of $\mathcal{F}$, contradicting Lemma 8.2.

We now check that extended $\mathcal{F}$-complexity is decreased and that (i)-(iv) of 6.3 are satisfied. It is clear that $\mathcal{H}^{\prime}$ respects $\mathcal{H}$. This is because (for $i=0$ and 1) each $i$-handle of $\mathcal{H}^{\prime}$ is an $i$-handle of $\mathcal{H}$ and inherits its product structure. Of course, $\mathrm{H}_{2}$ need not lie in any 2-handle of $\mathcal{H}$, but this was not 
a requirement of Definition 6.1. This explains why Definition 6.1 did not make more stringent requirements on 2-handles.

If $F$ is any component of $\mathcal{F}$ and $F^{\prime}=F \cap \mathcal{F}^{\prime}$, then $F^{\prime}$ is either a copy of $F$, or is completely removed, or is obtained by performing a sequence of the following operations: Remove a 0-handle of $F$ which abuts precisely two 1-handles of $F$, and amalgamate these two 1-handles into a single 1-handle of $F^{\prime}$. Hence, Lemma 7.2 ensures that Conditions (ii) and (iv) of 6.3 are satisfied and also that extended $\mathcal{F}$-complexity is reduced. Condition (iii) of 6.3 is clear.

We may therefore assume that $\mathcal{A}$ is an $I$-bundle over a surface $G$ other than a disc.

Case 2. $\partial_{v} \mathcal{A}=\emptyset$.

In other words, $G$ is a closed surface. If $\partial \mathcal{A}$ is entirely contained in $\mathcal{R}_{-}$or entirely contained in $\mathcal{R}_{+}$, then it has zero Euler characteristic, since $(M, \gamma)$ is taut and so $G$ is a torus or Klein bottle. In either case, we pick a nonseparating orientation-preserving curve in $G$, and perform a decomposition along the $I$-bundle over this curve. This cuts $\mathcal{A}$ into a solid torus. We perform one further taut decomposition along a product disc, ending with a single 3 -ball. We let this be a 0 -handle of $\mathcal{H}^{\prime}$. Suppose now that $\partial \mathcal{A}$ intersects both $\mathcal{R}_{-}$and $\mathcal{R}_{+}$. Then $\mathcal{A}$ must be a product $G \times I$. We can then perform a sequence of decompositions along product annuli and product discs, ending with a 3-ball, which again we let be a single 0 -handle of $\mathcal{H}^{\prime}$.

We therefore assume that $\partial_{v} \mathcal{A}$ is nonempty.

Case 3. Each annulus of $\partial_{v} \mathcal{A}$ is an incompressible product annulus.

Then by Lemma 4.2 of [10], the decomposition $(M, \gamma) \stackrel{\partial_{v} \mathcal{A}}{\longrightarrow}\left(M^{\prime}, \gamma^{\prime}\right)$ is taut. We perform this decomposition. In other words, we separate off $\mathcal{A}$ from $\mathcal{H}-\mathcal{A}$, and add sutures $\gamma^{\prime}$ as appropriate. By definition, $\mathcal{H}-\mathcal{A}$ is a handle structure.

The amalgam $\mathcal{A}$ does not inherit a handle structure (for example, 1handles of $\mathcal{A}$ need not be attached to 0 -handles of $\mathcal{A}$ ). However, since $\partial_{v} \mathcal{A}$ touches both $\mathcal{R}_{-}$and $\mathcal{R}_{+}$, the $\partial I$-bundle over $G$ cannot be connected, and so $\mathcal{A}$ must a product $G \times I$. As in Case 2, we may perform some further decompositions along product discs, which reduce $G \times I$ to a ball. We let this be a single 0 -handle of $\mathcal{H}^{\prime}$.

This whole procedure has the effect of removing some components of $\mathcal{F}$ and also replacing some 0 -handles and 1-handles of $\mathcal{F}$ with $\operatorname{arcs}$ of $\gamma^{\prime} \cap$ $\mathcal{H}^{0}\left(M^{\prime}\right)$. An argument almost identical to that in Procedure 3 of Section 7 establishes that the hypotheses of Lemma 7.2 hold. Therefore, (i)-(iv) of 6.3 hold and extended $\mathcal{F}$-complexity has been reduced.

We therefore assume that some annulus of $\partial_{v} \mathcal{A}$ is not an incompressible product annulus. 
Case 4. Some annulus $A$ of $\partial_{v} \mathcal{A}$ is compressible in $M$.

Then $A$ compresses in $M$ to two discs $D_{1}^{\prime}$ and $D_{2}^{\prime}$ with boundaries in $\mathcal{R}_{ \pm}$. Since $\mathcal{R}_{ \pm}$is incompressible in $M$ and $M$ is irreducible, $D_{1}^{\prime}$ and $D_{2}^{\prime}$ are parallel in $M$ to discs $D_{1}$ and $D_{2}$ in $\mathcal{R}_{ \pm}$. We pick $A$ so that the curve $\partial D_{1}$ is an innermost curve of $\partial_{v} \mathcal{A} \cap \mathcal{R}_{ \pm}$in $\mathcal{R}_{ \pm}$. Since $\mathcal{A}$ is not an $I$-bundle over a disc, this implies that $\operatorname{int}\left(D_{1}\right)$ is disjoint from $\mathcal{A}$. The parallelity region between the discs $D_{i}$ and $D_{i}^{\prime}$ is a ball $B_{i}$. Then, $B_{1}$ and $B_{2}$ are either disjoint or nested.

Case 4A. $B_{1}$ and $B_{2}$ are disjoint.

Then, $D_{1}$ and $D_{2}$ are disjoint, and the sphere $D_{1} \cup D_{2} \cup A$ bounds a ball $B$ in $M$. Since $\mathcal{A} \cap B=A$, we can extend the $I$-bundle structure of $\mathcal{A}$ over $B$. This contradicts the maximality of $\mathcal{A}$.

Case 4B. $B_{1}$ and $B_{2}$ are nested.

Then $B_{1} \subset B_{2}$ and $D_{1} \subset D_{2}$. The component $V$ of $M-\operatorname{int}(\mathcal{N}(A))$ lying wholly within $B_{2}$ is homeomorphic to the exterior of a knot in $S^{3}$. The amalgam $\mathcal{A}$ lies in $V$, and we may therefore remove $V$ from $M$ and still retain a handle structure. This does not change the homeomorphism type of $M$ and Lemma 7.3 gives that extended $\mathcal{F}$-complexity decreases and that (i)-(iv) of 6.3 hold.

Case 5. $\partial_{v} \mathcal{A}$ is incompressible, and some component of $\partial_{v} \mathcal{A}$ is not a product annulus.

Now, the $\partial I$-bundle over $G$ has at most two components. Therefore, if some component of $\partial_{v} \mathcal{A}$ is not a product annulus, then no component of $\partial_{v} \mathcal{A}$ is a product annulus. Let us suppose that $\partial_{v} \mathcal{A}$ is disjoint from $\mathcal{R}_{-}$ (say).

Pick any component $A$ of $\partial_{v} \mathcal{A}$. Then we let $A_{1}$ and $A_{2}$ be two parallel copies of $A$, incoherently oriented in such a way that the parallelity region $Y$ in $M^{\prime}=M-\operatorname{int}\left(\mathcal{N}\left(A_{1} \cup A_{2}\right)\right)$ inherits four sutures. Isotope $A_{1}$ and $A_{2}$ a little so that they become standard surfaces. Consider the decomposition $(M, \gamma) \stackrel{A_{1} \cup A_{2}}{\longrightarrow}\left(M^{\prime}, \gamma^{\prime}\right)$.

Case 5A. $\left(M^{\prime}, \gamma^{\prime}\right)$ is taut.

Then we perform this decomposition. We now check the requirements of 6.3 and also that extended $\mathcal{F}$-complexity has been reduced. We will use Lemma 7.2 to do this.

Let $\mathcal{H}^{\prime}$ be the handle structure which $\left(M^{\prime}, \gamma^{\prime}\right)$ inherits. Consider a component $F$ of $\mathcal{F}$ which is altered by this decomposition, and let $F^{\prime}$ be $F \cap \mathcal{F}^{\prime}$. By Lemma 8.2, $F$ must have had positive index.

Suppose that the extended $\mathcal{F}$-complexity of $F^{\prime}$ is at least that of $F$; we aim to reach a contradiction. We must have $C_{1}(X) \geq C_{1}(F)$ for some component $X$ of $F^{\prime}$. But each 1-handle of $F$ gives rise to precisely one 1-handle of $F^{\prime}$. Hence, $X$ must have all the 1-handles of $F^{\prime}$. 
Each component of $A \cap F$ yields three discs of $F^{\prime}$. Two of these discs have no 1-handles and intersect $\gamma^{\prime}$ four times. The remaining disc has at least one 1-handle, and has negative index. Since it has least one 1-handle, it must be $X$, and therefore $X$ has negative index. Therefore $C_{2}(X)<C_{2}(F)$. Hence, $C_{\mathcal{F}}^{+}\left(F^{\prime}\right)<C_{\mathcal{F}}^{+}(F)$. Lemma 7.2 now gives that (i), (ii) and (iv) of 6.3 hold, and that extended complexity has been reduced. Verifying (iii) of 6.3 is straightforward.

Case 5B. $\left(M^{\prime}, \gamma^{\prime}\right)$ is not taut.

Since $M$ is irreducible, so must $M^{\prime}$ be. Also, $\mathcal{R}_{ \pm}\left(M^{\prime}\right)$ is norm-minimising in $H_{2}\left(M^{\prime}, \gamma^{\prime}\right)$. For if $S$ is any surface in $M^{\prime}$ with $S \cap \partial M^{\prime}=\gamma^{\prime}$ and $[S, \partial S]=$ $\left[\mathcal{R}_{ \pm}\left(M^{\prime}\right), \gamma^{\prime}\right] \in H_{2}\left(M^{\prime}, \gamma^{\prime}\right)$, then $[S-Y, \partial S-Y]=\left[\mathcal{R}_{ \pm}\left(M^{\prime}\right)-Y, \gamma^{\prime}-Y\right]$ $=\left[\mathcal{R}_{ \pm}(M), \gamma\right] \in H_{2}(M, \gamma)$. So, $\chi_{-}(S) \geq \chi_{-}(S-Y) \geq \chi_{-}\left(\mathcal{R}_{ \pm}(M)\right)=$ $\chi_{-}\left(\mathcal{R}_{ \pm}\left(M^{\prime}\right)\right)$. Hence, the only way that $\left(M^{\prime}, \gamma^{\prime}\right)$ can fail to be taut is if $\mathcal{R}_{ \pm}\left(M^{\prime}\right)$ is compressible. This compression cannot reduce $\chi_{-}\left(\mathcal{R}_{ \pm}\left(M^{\prime}\right)\right)$, as $\mathcal{R}_{ \pm}\left(M^{\prime}\right)$ is norm-minimising. Hence, any compressible component of $\mathcal{R}_{ \pm}\left(M^{\prime}\right)$ is a torus or annulus. However, any circle in a compressible annulus is homotopically trivial in $M$. In particular, $A$ could not have been incompressible, contrary to assumption. Thus, if $\mathcal{R}_{ \pm}\left(M^{\prime}\right)$ is not taut, there are three cases to consider:

(i) Only one of $A_{1}$ and $A_{2}$ (say $A_{1}$ ) lies in a compressible torus component of $\mathcal{R}_{ \pm}\left(M^{\prime}\right)$ (called $T_{1}$, say) which disjoint from $\gamma^{\prime}$, or

(ii) $A_{1}$ and $A_{2}$ both lie in the same compressible torus $T_{1}$ disjoint from $\gamma^{\prime}$, or

(iii) $A_{1}$ and $A_{2}$ lie in distinct compressible tori $T_{1}$ and $T_{2}$ disjoint from $\gamma^{\prime}$.

Since $T_{i}$ is compressible and $M^{\prime}$ is irreducible, $T_{i}$ bounds a solid torus $V_{i}$ in $M^{\prime}$.

In Case (iii), the component of $M$ containing $A$ is the union of two solid tori, glued along an essential annulus. Thus, it is a Seifert fibre space with base space a disc and having at most two exceptional fibres (which are the cores of the solid tori). Also, it is disjoint from $\gamma$. Recall that, in the statement of 6.3 , we explicitly ruled out the case where it is has two exceptional fibres. If it has at most one exceptional fibre, it is a solid torus, and again, we ruled this case out.

In Case (ii), we pick the $A_{i}$ which is closest to $\mathcal{A}$. Then, the orientation of $A_{i}$ and $\mathcal{R}_{+} \cap \mathcal{A}$ agree near $\partial A_{i}$. The decomposition $(M, \gamma) \stackrel{A_{i}}{\longrightarrow}\left(M_{1}, \gamma_{1}\right)$ is taut. Exactly as in Case $5 \mathrm{~A}$, this reduces extended $\mathcal{F}$-complexity and (i)-(iv) of 6.3 are satisfied.

In Case (i), suppose first that $M-\operatorname{int}\left(\mathcal{N}\left(V_{1}\right)\right)$ contains $\mathcal{A}$. As above, the decomposition $(M, \gamma) \stackrel{A_{2}}{\longrightarrow}\left(M_{1}, \gamma_{1}\right)$ is taut, and, again, this reduces extended $\mathcal{F}$-complexity and (i)-(iv) of 6.3 are satisfied. 
Suppose now that $V_{1}$ contains $\mathcal{A}$. Then again the decomposition $(M, \gamma)$ $\stackrel{A_{2}}{\longrightarrow}\left(M_{1}, \gamma_{1}\right)$ is taut. This time one must work a little harder to verify that extended $\mathcal{F}$-complexity decreases and that (i)-(iv) of 6.3 are satisfied. Let $F$ be a component of $\mathcal{F}$, let $F^{\prime}=F \cap \mathcal{F}\left(M_{1}\right)$ and let $H_{0}$ be the 0-handle of $\mathcal{H}$ containing $F$. By Lemma 8.2, if $F$ is altered, then it must have positive index, and so it contributes towards $\mathcal{F}$-complexity. If $C_{\mathcal{F}}^{+}\left(F^{\prime}\right) \geq C_{\mathcal{F}}^{+}(F)$, then as in Case 5A, there must be a single component $X$ of $F^{\prime}$ containing all the 1-handles of $F^{\prime}$. Also, $X$ arises from a component of $F \cap A$. However, unlike in Case 5A, $X$ will not have negative index. In fact, it will be a disc which intersects $\gamma_{1}$ in four points. Hence, it has index two. Now, $F$ has positive index and therefore its index is at least two. Thus, if $C_{\mathcal{F}}^{+}\left(F^{\prime}\right) \geq C_{\mathcal{F}}^{+}(F)$, the index of $F$ is precisely two. If $F$ is an annulus intersecting $\gamma$ in two points, then $C_{3}(F)>C_{3}(X)$ and so $C_{\mathcal{F}}^{+}(F)>C_{\mathcal{F}}^{+}\left(F^{\prime}\right)$. Hence, we may assume that $F$ is a disc intersecting $\gamma$ in four points. In this case, all but two 0-handles of $F$ have valence two and are disjoint from $\gamma$. The two remaining 0-handles $D_{1}$ and $D_{2}$ each have valence one. These two handles contain a total of four points of $\gamma \cap F$. Since $\partial_{v} \mathcal{A}$ is disjoint from $\mathcal{R}_{-}$, each $D_{i}$ contains an even number of points of $\gamma \cap F$. If one of these 0-handles contains no points of $\gamma$, then it becomes a compression disc for $\mathcal{R}_{ \pm}\left(M_{1}\right)$, which contradicts the fact that $\left(M_{1}, \gamma_{1}\right)$ is taut. Hence, each $D_{i}$ contains precisely two points of $\gamma \cap F$. If these two points are joined by an arc of $\gamma \cap \partial H_{0}$, then again $\mathcal{R}_{ \pm}\left(M_{1}\right)$ is compressible, which is a contradiction. Therefore, for each $i$, the two points $\gamma \cap D_{i}$ are not joined by an arc of $\gamma \cap \partial H_{0}$.

Now, $F^{\prime}$ is $X$, together with two index zero discs. We remove the two index zero discs using Procedure 4. The component $X$ is a copy of $F$, and so $C_{\mathcal{F}}\left(F^{\prime}\right)=C_{\mathcal{F}}(F)$. If $F$ was not the only component of $\mathcal{F}$ in $H_{0}$, then these components of $\mathcal{F}$ have positive index, since otherwise we can apply one of Procedures 1-5. Hence, $n\left(\mathcal{H}^{\prime} \cap H_{0}\right)>1=n\left(H_{0}\right)$, where $n\left(\mathcal{H}^{\prime} \cap H_{0}\right)$ was defined in Section 5 to be the number of 0-handles of $\mathcal{H}^{\prime} \cap H_{0}$ containing a component of $\mathcal{F}^{\prime}$ of positive index. This implies that $C\left(H_{0} \cap \mathcal{H}^{\prime}\right)<C\left(H_{0}\right)$. If $F$ was the only component of $\mathcal{F}$ in $H_{0}$, then this is a trivial modification. This verifies (i)-(iv) of 6.3 .

We now need to check $C^{+}\left(\mathcal{H}^{\prime}\right)<C^{+}(\mathcal{H})$. But, if it is not, then the above must happen in every 0 -handle of $\mathcal{H}$ which is altered by the decomposition. This implies that the component of $M-\operatorname{int}\left(\mathcal{N}\left(V_{1}\right)\right)$ containing $A$ is a solid torus $V_{2}$ with $A \cap V_{2}$ a single annulus in $\partial V_{2}$ having winding number one. But $V_{1}$ is a solid torus, and so this component of $M$ is a solid torus, contradicting one of the assumptions of 6.3 . 


\section{Modifications to a decomposing surface.}

In the previous two sections, we performed a sequence of alterations to $\mathcal{H}$. We are now ready to tackle Proposition 6.4. Consider the taut decomposition $(M, \gamma) \stackrel{S}{\longrightarrow}\left(M_{S}, \gamma_{S}\right)$, where $S$ is a compact oriented surface properly embedded in $M$, having essential intersection with $\mathcal{R}_{ \pm}$. This implies that $S$ is taut and hence incompressible. Thus, by Lemmas 4.5 and 4.9 , we can assume that $S$ is in standard form in $\mathcal{H}$. But, as was remarked in Section 5 , there is a great deal of freedom over the form of $S \cap \partial \mathcal{H}^{0}$. The aim here is to perform a series of alterations to $S$, creating a new standard surface $S^{\prime}$ which has a considerably more restricted intersection with $\partial \mathcal{H}^{0}$. The sutured manifold obtained by decomposing $(M, \gamma)$ along $S^{\prime}$ will be written as $\left(M_{S^{\prime}}, \gamma_{S^{\prime}}\right)$.

Modification 1. Tubing along an arc.

Suppose that $\alpha$ is an arc in $\mathcal{R}_{ \pm}$with $\alpha \cap S=\partial \alpha$. Then there is an embedding of $\alpha \times[-1,1]$ in $\mathcal{R}_{ \pm}$with $\alpha \times\{0\}=\alpha$ and $(\alpha \times[-1,1]) \cap S=$ $\partial \alpha \times[-1,1]$. Suppose that the orientation that $\alpha \times[-1,1]$ inherits from $\mathcal{R}_{ \pm}$ agrees with the orientation of $S$ near $\partial \alpha \times[-1,1]$. Then we call $\alpha$ a tubing arc. We construct a new surface $S^{\prime}$ as follows: Embed $\alpha \times[-1,1] \times[0,1]$ in $M$ so that $(\alpha \times[-1,1] \times[0,1]) \cap \partial M=\alpha \times[-1,1] \times\{0\}=\alpha \times[-1,1]$ and $(\alpha \times[-1,1] \times[0,1]) \cap S=\partial \alpha \times[-1,1] \times[0,1]$. Then let

$$
\begin{aligned}
S^{\prime}= & S \cup(\alpha \times[-1,1] \times\{1\}) \\
& \cup(\alpha \times\{-1,1\} \times[0,1]) \\
& -(\partial \alpha \times(-1,1) \times[0,1)) .
\end{aligned}
$$

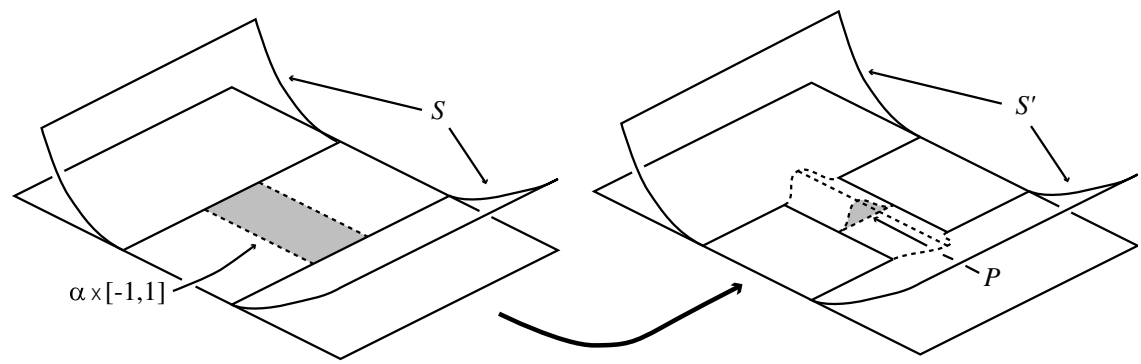

Figure 9.1.

We say that $S^{\prime}$ is obtained from $S$ by tubing along the arc $\alpha$. Note that if $\{*\}$ is a point in $\alpha-\partial \alpha$, then $P=\{*\} \times[-1,1] \times[0,1]$ is a product disc in $\left(M_{S^{\prime}}, \gamma_{S^{\prime}}\right)$. There is a commutative diagram: 


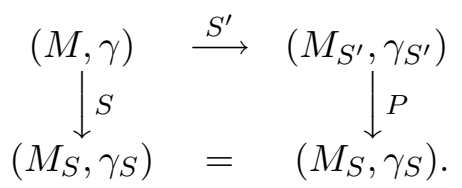

Hence, $\left(M_{S}, \gamma_{S}\right)$ is taut if and only if $\left(M_{S^{\prime}}, \gamma_{S^{\prime}}\right)$ is taut. However, $S^{\prime}$ need not have essential intersection with $\mathcal{R}_{ \pm}(M)$.

Modification 2. Slicing under an incompressible annulus.

Suppose that there is an annulus $A$ in $\mathcal{R}_{ \pm}$which is incompressible in $M$ and has $A \cap S=\partial A$. Let $A \times[0,1]$ be embedded in $M$, so that $(A \times[0,1]) \cap$ $\partial M=A \times\{0\}=A$, and so that $(A \times[0,1]) \cap S=\partial A \times[0,1]$. If the orientation of $A$ agrees with that of $S$ near $\partial A$, then we construct a new surface $S^{\prime}=S \cup(A \times\{1\})-(\partial A \times[0,1))$ by slicing under the incompressible annulus $A$. Let $C$ be a core circle of $A$. If we give $C \times[0,1]$ any orientation, then we have a commutative diagram:

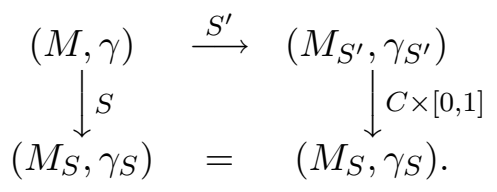

Since $A$ is incompressible in $M$, the annulus $C \times[0,1]$ is incompressible in $\left(M_{S^{\prime}}, \gamma_{S^{\prime}}\right)$, and so by 4.2 of $[\mathbf{1 0}],\left(M_{S}, \gamma_{S}\right)$ is taut if and only if $\left(M_{S^{\prime}}, \gamma_{S^{\prime}}\right)$ is taut.

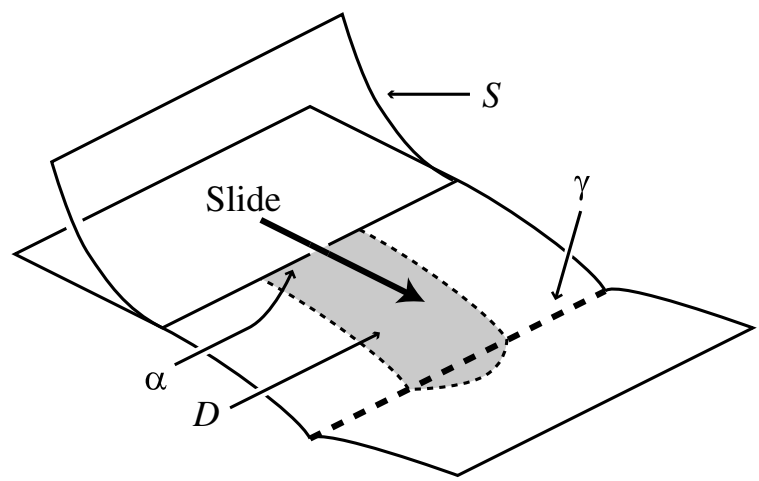

Figure 9.2.

Modification 3. Sliding $\partial S$ across $\gamma$.

Suppose that $D$ is a disc in $\partial M$, such that $D \cap S$ is an arc $\alpha$ in $\partial D$ and $D \cap \gamma$ is an arc properly embedded in $D$ disjoint from $\alpha$. Suppose that the orientation of $D$ near $\partial S$ agrees with the orientation of $S$. Let 
$D \times[0,1]$ be embedded in $M$ so that $(D \times[0,1]) \cap \partial M=D \times\{0\}=D$ and $(D \times[0,1]) \cap S=\alpha \times[0,1]$. Let

$$
\begin{aligned}
S^{\prime}= & S \cup \partial(D \times[0,1]) \\
& -(D-\partial D) \times\{0\} \\
& -(\alpha-\partial \alpha) \times[0,1) .
\end{aligned}
$$

(See Figure 9.2.) Then $\left(M_{S}, \gamma_{S}\right)$ and $\left(M_{S^{\prime}}, \gamma_{S^{\prime}}\right)$ are homeomorphic.

Modification 4. Slicing under a disc of contact.

Suppose that there is a disc $D$ in $\mathcal{R}_{ \pm}$with $D \cap S=\partial D$, with the orientation of $D$ matching that of $S$ near $\partial D$. Then $D$ is known as a disc of contact. Embed $D \times[0,1]$ in $M$, so that $(D \times[0,1]) \cap \partial M=D \times\{0\}=D$, and so that $(D \times[0,1]) \cap S=\partial D \times[0,1]$. The surface $S^{\prime}=S \cup(D \times\{1\})-(\partial D \times[0,1))$ is obtained from $S$ by slicing under the disc of contact.

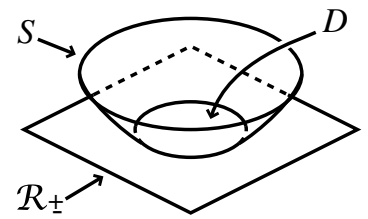

Figure 9.3.

Unfortunately, in this case, we have no guarantee that $\left(M_{S^{\prime}}, \gamma_{S^{\prime}}\right)$ is taut. But the following sequence of lemmas circumvents this. We introduce a temporary definition.

Definition 9.4. The surface $S$ in $(M, \gamma)$ is mountainous if some curve of $\partial S$ bounds a disc $D$ in $\mathcal{R}_{ \pm}$, such that the orientation of $D$ disagrees with that of $S$ near $\partial D$. The disc $D$ may also intersect $S$ away from $\partial D$.

Lemma 9.5. Suppose that $S$ is not mountainous. Let $S^{\prime}$ be obtained from $S$ by a sequence of Modifications 1, 2 and 3. Then $S^{\prime}$ is not mountainous.

Proof. It suffices to consider the case where $S^{\prime}$ is obtained from $S$ by a single modification of Type 1, 2 or 3. Consider first a modification of Type 1. Suppose that some curve of $\partial S^{\prime}$ bounds a disc $D$ in $\mathcal{R}_{ \pm}$, with orientation on $D$ disagreeing with that on $S^{\prime}$. Then at least one of the curves of $\alpha \times\{-1,1\}$ must lie in $\partial D$, since $S$ was not mountainous.

If both curves of $\alpha \times\{-1,1\}$ lie in $\partial D$, then the curves $\partial D \cup(\partial \alpha \times[-1,1])-$ $((\alpha-\partial \alpha) \times\{-1,1\})$ would have bounded the discs $D-((\alpha-\partial \alpha) \times[-1,1])$. Then $S$ would have been mountainous.

Suppose now that only one curve of $\alpha \times\{-1,1\}$ lies in $\partial D$, say $\alpha \times\{-1\}$. Then $\alpha \times\{1\}$ lies in $D-\partial D$, and so is part of a component of $\partial S^{\prime}$ bounding 
a disc $D^{\prime}$ in $\mathcal{R}_{ \pm}$. Then, $D-\left(D^{\prime}-\partial D^{\prime}\right)-((\alpha-\partial \alpha) \times[-1,1])$ is a disc which would have made $S$ mountainous.

Now consider the case where $S^{\prime}$ is obtained from $S$ by slicing under an incompressible annulus $A$. This has the effect of removing two curves of $\partial S$, neither of which bounded discs in $\mathcal{R}_{ \pm}$. Hence, in this case, $S^{\prime}$ is mountainous if and only if $S$ is mountainous.

Finally, consider the case where $S^{\prime}$ is obtained from $S$ by sliding an arc of $\partial S$ across $\gamma$. Then, this only creates new intersection points between the surface and $\gamma$, and so a curve of $\partial S^{\prime}$ disjoint from $\gamma$ is a copy of a curve of $\partial S$ disjoint from $\gamma$. Thus, if $S^{\prime}$ is mountainous, then so is $S$.

Lemma 9.6. Suppose that no component of $S$ is a disc disjoint from $\gamma$. Let $S^{\prime}$ be a surface obtained from $S$ by Modifications 1, 2 and 3. Then no component of $S^{\prime}$ is a disc disjoint from $\gamma$.

Proof. It suffices to consider a single modification of Type 1, 2 or 3 . If a component of $S^{\prime}$ which is a disc disjoint from $\gamma$ arises by tubing along an arc $\alpha$, then the components of $S$ containing $\partial \alpha$ were both discs disjoint from $\gamma$, contrary to assumption. If a disc component of $S^{\prime}$ arises by slicing under an annulus, then that annulus could not have been incompressible in $M$. A Type 3 modification cannot create components of $S^{\prime}$ disjoint from $\gamma$.

Lemma 9.7. Let $S$ be a surface in a taut sutured manifold $(M, \gamma)$ having essential intersection with $\mathcal{R}_{ \pm}$. Let $S_{2}$ be obtained from $S$ by a sequence of Modifications 1, 2 and 3, and let $S_{3}$ be obtained from $S_{2}$ by slicing under a disc of contact $D$. Then $S_{3}$ is in fact obtained from $S$ by a sequence of Modifications 1, 2 and 3.

Proof. We shall prove this by induction on the number $n$ of Type 1, 2 and 3 modifications from $S$ to $S_{2}$. For $n=0$, the statement of the lemma is empty, since $S=S_{2}$ has essential intersection with $\mathcal{R}_{ \pm}(M)$ and so has no discs of contact.

Suppose now the lemma is true for sequences of length at most $(n-1)$. Let $S_{1}$ be the surface obtained from $S$ by the first $(n-1)$ modifications. Then $S_{2}$ is obtained from $S_{1}$ by a modification of Type 1, 2 or 3 , and $S_{3}$ is obtained from $S_{2}$ by slicing under a disc of contact $D$.

Suppose that $S_{2}$ is obtained from $S_{1}$ by tubing along an $\operatorname{arc} \alpha$. Then, $D$ is disjoint from $\alpha \times(-1,1)$. If the disc $D$ is disjoint from $\alpha \times\{-1,1\}$, then we can obtain $S_{3}$ from $S_{1}$ by slicing under $D$, which gives a surface $S_{4}$ say, then tubing along $\alpha$. Inductively, $S_{4}$ is obtained from $S$ by Modifications 1 , 2 and 3 , and so the lemma is true in this case. Hence, we may assume that $D$ touches at least one of the $\operatorname{arcs} \alpha \times\{-1,1\}$. If it touches only one arc, then $S_{3}$ is ambient isotopic to $S_{1}$, and the lemma is true. If $D$ touches both arcs, then $D \cup(\alpha \times[-1,1])$ is an annulus $A$ in $\mathcal{R}_{ \pm}$. The two curves of $\partial A$ are boundary components of $S_{1}$. If $A$ is compressible in $M$, then both curves 
of $\partial A$ bound discs in $\mathcal{R}_{ \pm}$, since $(M, \gamma)$ is taut. One of these discs has an orientation disagreeing with that of $S_{1}$ near the boundary of the disc, and so $S_{1}$ is mountainous, contrary to Lemma 9.5. Hence, $A$ is incompressible in $M$. We may slice under $A$ to obtain $S_{3}$ from $S_{1}$. This proves the lemma in this case.

Suppose that $S_{2}$ is obtained from $S_{1}$ by slicing under an incompressible annulus $A$. Then $A$ cannot lie in $D$, since $A$ is incompressible. Hence, we can obtain $S_{3}$ from $S_{1}$ by slicing under $D$, then slicing under $A$. The inductive hypothesis proves the lemma.

Similarly, if $S_{2}$ is obtained from $S_{1}$ by sliding an arc of $\partial S_{1}$ across $\gamma$, then the relevant component of $\partial S_{2}$ is disjoint from $D$, and therefore we may obtain $S_{3}$ from $S_{1}$ by slicing under $D$, then performing the Type 3 modification. Again, the inductive hypothesis proves the lemma.

Corollary 9.8. Let $S$ be a surface in the taut sutured manifold $(M, \gamma)$ having essential intersection with $\mathcal{R}_{ \pm}$. Then any surface obtained from $S$ by Modifications 1, 2, 3 and 4 is in fact obtained from $S$ by Modifications 1, 2 and 3 .

Unfortunately, if $S^{\prime}$ is a surface created from $S$ by Modifications 1, 2 and 3 , then $S^{\prime}$ need not be incompressible. The incompressibility of $S$ was useful in showing that $S$ can be isotoped into standard form. We therefore need the following lemma:

Lemma 9.9. Let $S$ be a surface in $(M, \gamma)$, having essential intersection with $\mathcal{R}_{ \pm}$. Suppose that $(M, \gamma) \stackrel{S}{\longrightarrow}\left(M_{S}, \gamma_{S}\right)$ is taut. Let $S^{\prime}$ be a surface obtained from $S$ by a sequence of Modifications 1, 2 and 3 . If $S^{\prime}$ is in vertical form with respect to some handle structure on $(M, \gamma)$, then we may perform an ambient isotopy of $S^{\prime}$ and perhaps some Type 4 modifications, taking $S^{\prime}$ into standard form, without increasing its complexity.

Proof. Consider again the proof of Lemma 4.9. The crucial property of $S$ was that if $D$ is any disc in $M-\partial M$ with $D \cap S=\partial D$, then $\partial D$ bounds a disc $D^{\prime}$ in $S$, and we may ambient isotope $D^{\prime}$ onto $D$. In fact, we need only restrict attention to discs $D$ lying in a single 0-handle of $M$. In the case of $S^{\prime}$, the circle $\partial D$ need not bound a disc in $S^{\prime}$, since $S^{\prime}$ might not be incompressible. But, since $\left(M_{S^{\prime}}, \gamma_{S^{\prime}}\right)$ is taut, $\partial D$ bounds a disc $D^{\prime}$ in $\mathcal{R}_{ \pm}\left(M_{S^{\prime}}\right)$. Consider a circle $C$ of $D^{\prime} \cap \partial S^{\prime}$ innermost in $D^{\prime}$. By Lemma 9.6, this cannot bound a disc of $S^{\prime}$. Hence, it bounds a disc of contact in $\mathcal{R}_{ \pm}(M)$. Slice under this disc of contact. By Corollary 9.8, the new surface (also called $S^{\prime}$ ) is in fact obtained from $S$ by a sequence of Modifications 1, 2 and 3. Hence, the new $\left(M_{S^{\prime}}, \gamma_{S^{\prime}}\right)$ is taut. So, we may repeat this process and, in this way, we may remove all circles of $D^{\prime} \cap \partial S^{\prime}$. But then $\partial D$ bounds a disc $D^{\prime}$ in $S^{\prime}$, and we may ambient isotope $D^{\prime}$ onto $D$. The new surface $S^{\prime}$ is obtained from the old $S^{\prime}$ by removing $S^{\prime} \cap\left(D^{\prime}-\partial D^{\prime}\right)$ and gluing in $D$. Thus, the complexity 
of the new $S^{\prime}$ is no more than the complexity of the old $S^{\prime}$. Continuing in this fashion, we may get $S^{\prime}$ into standard form.

We will alter $S$, using Modifications 1, 2, 3 and 4, until $S$ has become a standard surface satisfying each of the following three conditions:

1. Each curve of $S \cap \partial \mathcal{H}^{0}$ meets any 1-handle of $\mathcal{F}$ in at most one arc.

2. There exists no tubing arc in $\partial \mathcal{F}^{0} \cap \mathcal{R}_{ \pm}$.

3. Suppose that $D$ is a disc in $\mathcal{F}^{0}$ with $\partial D$ the union of two arcs $\alpha$ and $\beta$, where $\alpha=S \cap \partial D$ and $\beta=D \cap \partial \mathcal{F}^{0}$. Suppose that one endpoint of $\alpha$ lies in $\mathcal{R}_{ \pm}$and one endpoint lies in $\mathcal{F}^{1}$. Then at least one of the following must happen:

- $\beta$ touches at least two components of $\partial \mathcal{F}^{0} \cap \partial \mathcal{F}^{1}$,

- $\beta$ touches $\gamma$ and the orientation of $\alpha$ and $\beta$ disagree locally near $\alpha \cap \beta \cap \mathcal{R}_{ \pm}$, or

- $\beta$ touches $\gamma$ at least twice, and the orientation of $\alpha$ and $\beta$ agree locally near $\alpha \cap \beta \cap \mathcal{R}_{ \pm}$.

Diagrams clarifying Conditions 1, 2 and 3 are given in Figures 9.10, 9.11 and 9.12. The alterations to $S$ which ensure that Conditions 1,2 and 3 hold will reduce its complexity, and so they are guaranteed to terminate. The above three conditions are not quite sufficient for our purposes. We also wish to ensure that the following two conditions hold:

4. Each curve of $S \cap \partial \mathcal{H}^{0}$ meets any component of $\mathcal{R}_{ \pm} \cap \partial \mathcal{H}^{0}$ in at most one arc.

5. If $\alpha$ is an arc of $S \cap \mathcal{F}^{0}$ with both endpoints in $\mathcal{R}_{ \pm}$, then each of the two arcs in $\partial \mathcal{F}^{0}$ joining $\partial \alpha$ must either touch $\partial \mathcal{F}^{1}$ or hit $\gamma_{S}$ more than twice.

A diagram clarifying Condition 5 is given in Figure 9.17. To achieve Conditions 4 and 5, we will need two further types of modification to $S$, which we will describe later. We now show that Conditions 1-3 can be achieved. By Lemma 4.9, we may assume that $S$ is in standard form. Each alteration to $S$ leaves it in vertical form, but not necessarily standard form. However, we can then use Lemma 9.9 to get $S$ into standard form, since the alterations to $S$ used there result in the removal of some components of $S \cap \partial \mathcal{H}^{0}$, and hence the new $S$ also satisfies Conditions 1-3.

Condition 1. Each curve of $S \cap \partial \mathcal{H}^{0}$ meets any 1-handle of $\mathcal{F}$ in at most one arc.

Suppose that this condition does not hold. We will construct a ball $B$ lying in $M-\partial M$, such that $B \cap S$ is a disc in $\partial B$. We will then ambient isotope $S$ across $B$, and in doing so, reduce the complexity of $S$.

By assumption, there is a curve $C$ of $S \cap \partial \mathcal{H}^{0}$ containing two sub-arcs $\alpha_{1}$ and $\alpha_{2}$, which are both properly embedded in the same 1-handle $D_{1}$ of $\mathcal{F}$. Pick $C$ to be a curve of $S \cap \partial \mathcal{H}^{0}$ which is innermost in $\partial \mathcal{H}^{0}$ amongst all 


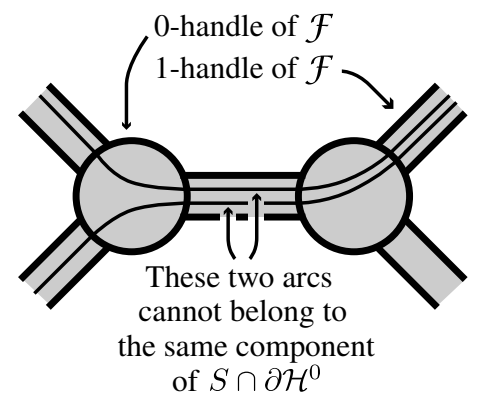

Figure 9.10.

curves with this property. Since $S$ is standard, there is a disc $D_{2}$ of $S \cap \mathcal{H}^{0}$ with $\partial D_{2}=C$. Let $H_{0}$ (respectively, $H_{2}$ ) be the 0-handle (respectively, 2-handle) containing $\alpha_{1}$ and $\alpha_{2}$, and let $E_{1}$ and $E_{2}$ be the discs of $H_{2} \cap S$ containing $\alpha_{1}$ and $\alpha_{2}$. By the 'innermost' assumption on $C$, the two arcs $\alpha_{1}$ and $\alpha_{2}$ are adjacent in $D_{1}$, in the sense that no other arcs of $S \cap D_{1}$ lie between them. Let $B^{\prime}$ be the closure of the component of $H_{2}-\left(E_{1} \cup E_{2}\right)$ lying between $E_{1}$ and $E_{2}$. The ball $B^{\prime}$ will be part of $B$.

Let $\beta_{1}$ be an arc in the interior of $D_{1}$ which runs from $\alpha_{1}$ to $\alpha_{2}$, but which intersects $S$ in no other points. Let $\beta_{2}$ be an arc properly embedded in $D_{2}$, with $\partial \beta_{2}=\partial \beta_{1}$. Then $\beta_{1} \cup \beta_{2}$ bounds a disc $D_{3}$ in $H_{0}$. We can assume that $D_{3}-\partial D_{3}$ misses $S$ and $\partial H_{0}$. Let $B$ be a small neighbourhood of $D_{3} \cup B^{\prime}$ in $M$. Then an ambient isotopy of $S$ across $B$ has the effect of reducing $\left|S \cap \mathcal{H}^{2}\right|$, by removing the discs $E_{1}$ and $E_{2}$. The new surface is a vertical surface with lower complexity than that of $S$.

Condition 2. There exists no tubing arc in $\partial \mathcal{F}^{0} \cap \mathcal{R}_{ \pm}$.

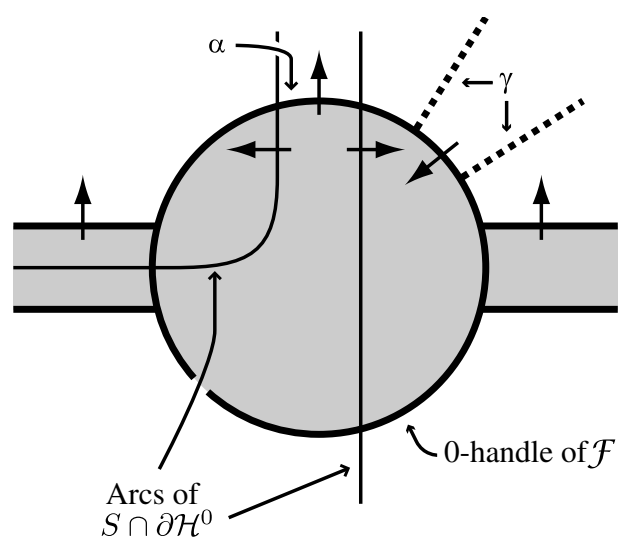

Figure 9.11. 
Suppose that $\alpha$ is such an arc. We will tube $S$ along $\alpha$. Let $H_{1}$ be the 1handle of $\mathcal{H}$ containing $\alpha$. We may pick $\alpha \times[-1,1]$ so that $(\alpha \times[-1,1]) \cap H_{1}$ is vertical in $H_{1}$, and so that $\operatorname{cl}\left((\alpha \times[-1,1])-H_{1}\right)$ is two small discs in $\mathcal{H}^{0}$. Then the surface $S^{\prime}$ constructed from $S$ by tubing along $\alpha$ has lower complexity than that of $S$, since $\left|S \cap \mathcal{H}^{2}\right|=\left|S^{\prime} \cap \mathcal{H}^{2}\right|$, and $\left|\partial S^{\prime} \cap \mathcal{H}^{1}\right|=\left|\partial S \cap \mathcal{H}^{1}\right|-2$.

Condition 3. Suppose that $D$ is a disc in $\mathcal{F}^{0}$ with $\partial D$ the union of two arcs $\alpha$ and $\beta$, where $\alpha=S \cap \partial D$ and $\beta=D \cap \partial \mathcal{F}^{0}$. Suppose that one endpoint of $\alpha$ lies in $\mathcal{R}_{ \pm}$and one endpoint lies in $\mathcal{F}^{1}$. Then at least one of the following must happen:

- $\beta$ touches at least two components of $\partial \mathcal{F}^{0} \cap \partial \mathcal{F}^{1}$,

- $\beta$ touches $\gamma$ and the orientation of $\alpha$ and $\beta$ disagree locally near $\alpha \cap$ $\beta \cap \mathcal{R}_{ \pm}$, or

- $\beta$ touches $\gamma$ at least twice, and the orientation of $\alpha$ and $\beta$ agree locally near $\alpha \cap \beta \cap \mathcal{R}_{ \pm}$.

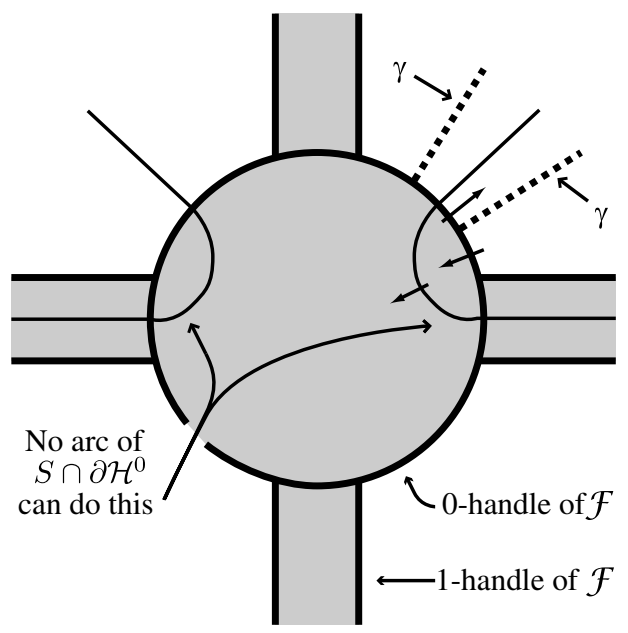

Figure 9.12.

Suppose that $D$ is such a disc but that it fails to satisfy each of the three alternatives of Condition 3. In particular, $\beta$ touches only one component of $\partial \mathcal{F}^{0} \cap \partial \mathcal{F}^{1}$ (which is therefore the component of $\partial \mathcal{F}^{0} \cap \partial \mathcal{F}^{1}$ which contains an endpoint of $\alpha$ ). Suppose also that there is no sub-arc of $\beta$ which violates Condition 2. Let $H_{1}=D^{2} \times I$ be the 1-handle of $\mathcal{H}$ containing $D$.

There are a number of cases to consider. Suppose first that $\beta \cap \gamma=\emptyset$. Then, let $B$ be the vertical ball $D \times I$ in $H_{1}$. Let $D^{\prime}$ be the disc of $S \cap \mathcal{H}^{2}$ which touches $\alpha$ at a single point. Let $B^{\prime}$ be the closure of the component of $\mathcal{H}^{2}-D^{\prime}$ which has nonempty intersection with $\beta-\partial \beta$. There is an ambient isotopy of $S$ across the ball $B \cup B^{\prime}$, leaving $S$ in vertical form, and reducing 
$\left|S \cap \mathcal{H}^{2}\right|$. This isotopy will also move parts of $S$ lying in $D \times I$, but this causes no problems.

Suppose now that $\beta \cap \gamma \neq \emptyset$. Then, by assumption, the orientations of $\alpha$ and $\beta$ agree near $\alpha \cap \beta \cap \mathcal{R}_{ \pm}$, and also $\beta \cap \gamma$ is a single point. Suppose first that there is no arc of $S \cap \mathcal{F}^{0}$ other than $\alpha$ lying in $D$. Then we perform a Type 3 modification, supported in a small neighbourhood of $H_{1}$, which slides the vertical arc $\left(\beta \cap \alpha \cap \mathcal{R}_{ \pm}\right) \times I$ in $H_{1}$ across the vertical arc $(\beta \cap \gamma) \times I$. Then, we can perform the ambient isotopy described above to reduce the complexity of $S$.

Suppose now that there exists some arc of $S \cap \partial \mathcal{F}^{0}$ other than $\alpha$ lying in $D$. Let $\alpha_{1}$ be the arc adjacent to $\alpha$. If the sub-arc $\beta_{1}$ of $\beta$ lying between $\alpha$ and $\alpha_{1}$ is a tubing arc, then Condition 2 is violated. If $\beta_{1}$ is not a tubing arc, then there are two possibilities: $\beta_{1}$ touches $\gamma$, or the orientations of $\alpha_{1}$ and $\beta_{1}$ disagree near $\alpha_{1} \cap \beta_{1}$. Applying this argument to each arc of $S \cap D$ with an endpoint lying between $\beta \cap \alpha \cap \mathcal{R}_{ \pm}$and $\beta \cap \gamma$, we see that these arcs are all coherently oriented. In particular, we can slide each of these arcs across $\gamma$. Then, we can apply the ambient isotopy described above.

Thus, we may ensure that $S$ satisfies Conditions 1,2 and 3 . We now ensure that $S$ also satisfies Condition 4 and 5. To do this, we will need two further modifications.

Modification 5. Surgery along a product disc.

This is defined to be the reverse of a Type 1 modification.

Modification 6. Removal of a product region.

Suppose that a component $F_{1}$ of $S$ is parallel to a surface $F_{2}$ in $\mathcal{R}_{ \pm}$, and the orientations of $F_{1}$ and $F_{2}$ disagree near $\partial F_{1}=\partial F_{2}$. Suppose also that the interior of the parallelity region between $F_{1}$ and $F_{2}$ is disjoint from $S$. If we remove $F_{1}$ from $S$, creating a new surface $S^{\prime}$, then $\left(M_{S}, \gamma_{S}\right)$ is homeomorphic to $\left(M_{S^{\prime}}, \gamma_{S^{\prime}}\right)$, plus a product component $\left(F_{1} \times[0,1], \partial F_{1} \times\{0\}\right)$. Hence, $\left(M_{S^{\prime}}, \gamma_{S^{\prime}}\right)$ is taut if and only if $\left(M_{S}, \gamma_{S}\right)$ is taut.

We need some lemmas to ensure that Modifications 5 and 6 are well behaved.

Lemma 9.13. Let $S$ be a surface in $(M, \gamma)$, and let $S_{2}$ be a surface obtained from $S$ by a sequence of $n$ Type 5 modifications. Let $S_{3}$ be obtained from $S_{2}$ by slicing under a disc of contact. Then in fact, $S_{3}$ is obtained from $S$ by a sequence of Type 4 modifications, and then at most $n$ Type 5 modifications.

Proof. This is by induction on $n$. For $n=0$, the lemma is trivial. Therefore, assume that the lemma is true for less than $n$ Type 5 modifications, and let $S_{1}$ be the surface obtained from $S$ by the first $(n-1)$ Type 5 modifications. Let $\alpha$ be the tubing arc for $S_{2}$. If the disc of contact $D$ is disjoint from $\alpha$, then we may slice under $D$ before doing the Type 5 move, and so the lemma is true 
by induction. If $D$ is not disjoint from $\alpha$, then $D_{1} \cup D_{2}=D-(\alpha \times(-1,1))$ is two discs of contact for $S_{1}$, and $S_{3}$ is obtained by slicing under both $D_{1}$ and $D_{2}$. Applying the inductive hypothesis twice proves the lemma.

Lemma 9.14. Let $S$ be a surface in $(M, \gamma)$. Let $S_{2}$ be a surface obtained from $S$ by a sequence of Type 5 modifications, and let $S_{3}$ be a surface obtained from $S_{2}$ by a Type 6 modification. Then $S_{3}$ is obtained from $S$ by at most one Type 6 modification, then perhaps some Type 5 modifications.

Proof. We will prove this by induction on the number $n$ of Type 5 modifications from $S$ to $S_{2}$. For $n=0$, the statement of the lemma is empty. So, assume that the statement is true for sequences of length at most $(n-1)$. Let $S_{1}$ be the surface obtained from $S$ by the first $(n-1)$ Type 5 modifications. The surface $S_{1}$ is obtained from $S_{2}$ by tubing along an arc $\alpha$. Let $F_{1}$ be the component of $S_{2}$ which we remove in the Type 6 modification. If neither component of $\partial \alpha$ lies in $F_{1}$, then we may obtain $S_{3}$ from $S_{1}$ by performing the Type 6 modification, then the Type 5 modification. The lemma is then true by induction. If both components of $\partial \alpha$ lie in $F_{1}$, then a single Type 6 modification takes $S_{1}$ to $S_{3}$, and again the lemma is true by induction. If a single component of $\partial \alpha$ lies in $F_{1}$, then we find a (possibly empty) collection of properly embedded arcs in $F_{1}$ which cut it to a disc. These arcs define Type 5 moves which can be applied to $S_{1}$, at the end of which we obtain a surface ambient isotopic to $S_{3}$. Hence, in this case also, the lemma is true.

Lemma 9.15. Let $S$ be a surface in $(M, \gamma)$ which is not mountainous. Let $S^{\prime}$ be obtained from $S$ by a sequence of Type 6 modifications, then by slicing under a disc of contact $D$. Then $S^{\prime}$ is obtained from $S$ by a Type 4 modification, then some Type 6 modifications.

Proof. Suppose that some component $F_{1}$ of $S$ has $\partial F_{1}$ lying in $\operatorname{int}(D)$. Then it must be removed by some Type 6 modification. In particular, it must be parallel to a subsurface $F_{2}$ of $D$. The outermost component of $\partial F_{2}$ in $D$ is a component of $\partial S$ which makes $S$ mountainous, contrary to assumption. Hence, each component of $S$ is disjoint from $\operatorname{int}(D)$, and we may therefore slice under $D$ before performing the Type 6 modifications.

The above lemmas all give the following proposition:

Proposition 9.16. Let $S$ be a taut surface in the taut sutured manifold $(M, \gamma)$, with $S$ having essential intersection with $\mathcal{R}_{ \pm}$. Let $S^{\prime}$ be obtained from $S$ firstly by a (possibly empty) sequence of Modifications 1, 2, 3 and 4, then by a (possibly empty) sequence of Modifications 3, 4, 5 and 6. Then $S^{\prime}$ is in fact obtained from $S$ by a (possibly empty) sequence of Modifications 1 , 2 and 3 , then possibly by some Type 6 modifications, then possibly some Type 3 and 5 modifications. In particular, no Type 4 modifications are needed. 
Proof. Consider the sequence of numbers from 1 to 6 which are the type of each modification. Ignore repetitions; for example, if we perform two Type 3 modifications in a row, then only write down one 3 . Lemma 9.13 implies that if we write down 54, we may replace this with 45 or 4 . Lemma 9.14 implies that if we write down 56, we may instead write down $65,5,6$ or nothing. Also, we may replace 34 with 43, since each slide across $\gamma$ creates a component of $\partial S$ touching $\gamma$, whereas each slice under a disc of contact deals with a component of $\partial S$ disjoint from $\gamma$. Hence, we can perform the Type 4 modifications before the Type 3 modifications. Similarly, we can replace 36 with 63 . Hence, in the sequence of 3, 4, 5 and 6 modifications, we can arrange to do all the Type 5 and 3 modifications last. Corollary 9.8 asserts that we may replace the initial sequence of $1,2,3$ and 4 with just a sequence of 1,2 and 3 . Let $S_{1}$ be the surface obtained from $S$ after this initial sequence. Then, the sequence of numbers after $S_{1}$ is a (possibly empty) sequence of 4 and 6 (starting with 6 ), and then possibly a sequence of 5 and 3 . If the sequence of 6 and 4 is empty or a single 6 , the proposition is proved. Otherwise, the sequence of 6 and 4 starts with 64. By Lemma 9.5, $S_{1}$ is not mountainous and so, by Lemma 9.15, we may replace the 64 with 46. We may include the 4 in the initial sequence of 1, 2 and 3. Proceeding in this way, we prove the proposition.

We have so far modified $S$ using Modifications 1, 2, 3 and 4, resulting in a surface satisfying Conditions 1,2 and 3. We are now going to make some further alterations, using Modifications 3, 4, 5 and 6, resulting in a surface $S^{\prime}$ which also satisfies Conditions 4 and 5 . The point behind the above proposition is that we can in fact obtain $S^{\prime}$ from $S$ without slicing under discs of contact.

Condition 4. Each curve of $S \cap \partial \mathcal{H}^{0}$ meets any component of $\mathcal{R}_{ \pm} \cap \partial \mathcal{H}^{0}$ in at most one arc.

Suppose that, on the contrary, there are two arcs $\alpha_{1}$ and $\alpha_{2}$ of $S \cap \mathcal{R}_{ \pm} \cap \partial \mathcal{H}^{0}$ properly embedded in a component of $\mathcal{R}_{ \pm} \cap \partial \mathcal{H}^{0}$, such that $\alpha_{1}$ and $\alpha_{2}$ belong to the same component of $S \cap \partial \mathcal{H}^{0}$. We may assume that there is an $\operatorname{arc} \beta$ in $\mathcal{R}_{ \pm} \cap \partial \mathcal{H}^{0}$ with one endpoint in $\alpha_{1}$, one endpoint in $\alpha_{2}$, and the remainder of $\beta$ disjoint from $S$. There is also a disc $D$ in $\mathcal{H}^{0}$ with $\partial D$ containing $\beta$, and $D \cap S=\operatorname{cl}(\partial D-\beta)$.

Suppose first that $\beta$ is a tubing arc and hence that $D$ is disjoint from $\gamma_{S}$. Since $\mathcal{R}_{ \pm}\left(M_{S}\right)$ is incompressible, $\partial D$ bounds a disc $D^{\prime}$ in $\mathcal{R}_{ \pm}\left(M_{S}\right)$. If $\partial S \cap D^{\prime}$ is a single arc, then we may ambient isotope $S \cap D^{\prime}$ onto $D$. It is straightforward to verify that the resulting surface $S^{\prime}$ is standard and still satisfies Conditions 1-3. This procedure does not increase $\left|S \cap \mathcal{H}^{2}\right|$ and it decreases $\left|\partial S \cap \mathcal{H}^{1}\right|$. Hence, it decreases the complexity of $S$.

We must deal with the case where $\partial S \cap D^{\prime}$ contains some circles. Pick one $C$ innermost in $D^{\prime}$, bounding a disc $E$ in $D^{\prime}$. Then $E$ is either a disc 
of contact or a disc component of $S$. In the former case, we slice under the disc of contact. We now give a procedure for dealing with the latter case. The curve $C$ bounds a disc $E^{\prime}$ in $\mathcal{R}_{ \pm}(M)$, and $E \cup E^{\prime}$ bounds a ball $B$ in $M$. Pick a curve $C^{\prime}$ of $E^{\prime} \cap \partial S$ innermost in $E^{\prime}$. Then we may apply either Modification 4 or Modification 6 to the component of $S$ containing $C^{\prime}$. In this way, we eventually remove all components of $S$ lying in $B$. We can then apply Modification 6 to $E$. Continuing in this fashion, we eventually remove all circles of $\partial S \cap D^{\prime}$. Then we may ambient isotope $S \cap D^{\prime}$ onto $D$.

Suppose now that $\beta$ is not a tubing arc, in which case the $\operatorname{disc} D$ is a product disc in $M_{S}$. Let $D \times[-1,1]$ be embedded in $\mathcal{H}^{0}$, so that

- $D \times\{0\}=D$,

- $(D \times[-1,1]) \cap \gamma=\emptyset$,

- $(D \times[-1,1]) \cap \mathcal{R}_{ \pm}(M)=\beta \times[-1,1]$,

- $(D \times[-1,1]) \cap S=\operatorname{cl}(\partial D-\beta) \times[-1,1]$.

Let $S^{\prime}$ be $S-(\operatorname{cl}(\partial D-\beta) \times(-1,1)) \cup(D \times\{-1,1\})$. Let $\{*\}$ be a point in $\beta-\partial \beta$. Then $S$ is obtained from $S^{\prime}$ by tubing along the arc $\{*\} \times[-1,1]$. Hence, $S^{\prime}$ is obtained from $S$ by a Type 5 modification. It is straightforward to check that $S^{\prime}$ still satisfies Conditions 1,2 and 3. This procedure leaves the complexity of $S$ unchanged. It also creates two discs of $S^{\prime} \cap \mathcal{H}^{0}$ from one disc of $S \cap \mathcal{H}^{0}$. Each of the new discs either touches $\gamma$ or touches $\mathcal{H}^{1}$. But $S \cap \gamma=S^{\prime} \cap \gamma$ and $S \cap \mathcal{H}^{1}=S^{\prime} \cap \mathcal{H}^{1}$. Hence, eventually, this process terminates.

Condition 5. If $\alpha$ is an arc of $S \cap \mathcal{F}^{0}$ with both endpoints in $\mathcal{R}_{ \pm}$, then each of the two arcs in $\partial \mathcal{F}^{0}$ joining $\partial \alpha$ must either touch $\partial \mathcal{F}^{1}$ or hit $\gamma_{S}$ more than twice.

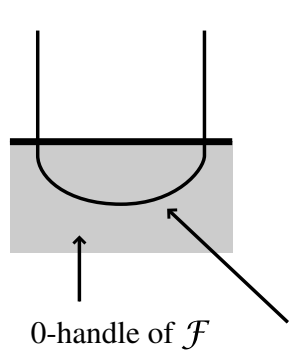

Forbidden arcs of $S \cap \partial \mathcal{H}^{0}$
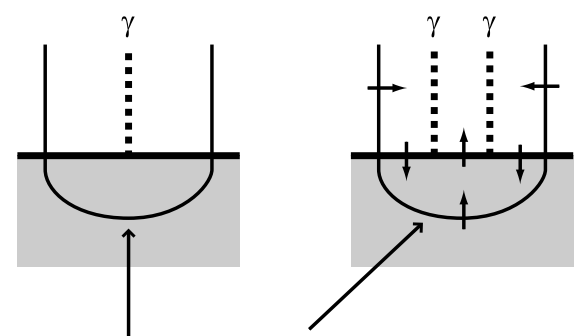

Figure 9.17.

Let $\alpha$ be such an arc. Suppose that there is an arc $\beta$ in $\partial \mathcal{F}^{0}$ joining the endpoints of $\alpha$, such that $\beta$ is disjoint from $\mathcal{F}^{1}$ and touches $\gamma_{S}$ at most twice. Let $D$ be the disc of $\mathcal{F}^{0}$ containing $\alpha$. Then, since Condition 2 holds, we may take $\alpha$ to be extrememost in $D$, separating off a disc $D^{\prime}$ from $D$ with $D^{\prime} \cap S=\alpha$. Then $D^{\prime}$ is a disc properly embedded in $M_{S}$. Hence, $\partial D^{\prime}$ hits 
$\gamma_{S}$ either twice or not at all. If $\partial D^{\prime}$ is disjoint from $\gamma_{S}$, then $\beta$ is a tubing arc, contrary to Condition 2.

Suppose therefore that $\partial D^{\prime}$ hits $\gamma_{S}$ at precisely two points. If both of these points lie at the endpoints of $\alpha$, then we will apply Modification 5 . If $H_{1}=D \times D^{1}$ is the 1 -handle containing $\alpha$, then we will take the tubing arc to be a slight extension of $\{*\} \times D^{1}$, where $\{*\}$ is a point in $\beta$. The result of this Type 5 modification is to leave $\left|S \cap \mathcal{H}^{2}\right|$ unchanged and to reduce $\left|\partial S \cap \mathcal{H}^{1}\right|$ by 2 . Hence, it reduces the complexity of $S$.

If there is a point $P$ of $\gamma_{S} \cap \partial D^{\prime}$ not lying at an endpoint of $\alpha$, then $P$ lies on $\gamma$, and we can perform a Type 3 modification sliding $\alpha \times D^{1}$ across $P \times D^{1}$. This slide leaves the complexity of $S$ unchanged. After possibly performing this operation once more, we end with a situation where both points of $\gamma_{S} \cap \partial D^{\prime}$ lie at the endpoints of $\alpha$. Hence, we may apply Modification 5 to reduce the complexity of $S$. It is clear that, in the above procedure, we have not violated Conditions 1-3.

\section{Behaviour of handle complexity under decomposition.}

The aim of this section is to complete the proof of Proposition 6.4. Recall that we are given a taut decomposition $(M, \gamma) \stackrel{S}{\longrightarrow}\left(M_{S}, \gamma_{S}\right)$, where $S$ has essential intersection with $\mathcal{R}_{ \pm}(M)$. Also, $(M, \gamma)$ is equipped with a handle structure $\mathcal{H}$, for which each 0 -handle of $\mathcal{F}=\mathcal{F}(\mathcal{H})$ has positive index.

In the previous section, we performed a sequence of alterations to $S$, resulting in a new standard surface (called $S^{\prime}$, say) satisfying Conditions 1-5. Let $(M, \gamma) \stackrel{S^{\prime}}{\longrightarrow}\left(M^{\prime}, \gamma^{\prime}\right)$ be the decomposition along $S^{\prime}$ and let $\mathcal{H}^{\prime}$ be the induced handle structure on $M^{\prime}$. Note that $[S, \partial S]=\left[S^{\prime}, \partial S^{\prime}\right] \in$ $H_{2}(M, \partial M)$.

Proposition 9.16 asserted that it sufficed to perform a sequence of Modifications 1, 2 and 3, then some Type 6 modifications, then some Type 3 and 5 modifications. If a Type 1 or 2 modification to $S$ results in a surface $S_{1}$, then there is a pull-back $\left(M_{S}, \gamma_{S}\right) \stackrel{P}{\longleftarrow}\left(M_{S_{1}}, \gamma_{S_{1}}\right)$, where $P$ is a product disc or incompressible product annulus. Hence, the sequence of Modifications 1, 2, 3 and 6 gives rise to the sequence of pull-backs

$$
(M, \gamma)=\left(\hat{M}_{1}, \hat{\gamma}_{1}\right) \stackrel{P_{1}}{\longleftarrow} \cdots \stackrel{P_{r-1}}{\longleftarrow}\left(\hat{M}_{r}, \hat{\gamma}_{r}\right)
$$

which was mentioned in Proposition 6.4. Then Modifications 3 and 5 give the sequence of decompositions

$$
\left(\hat{M}_{r}, \hat{\gamma}_{r}\right) \stackrel{P_{r}}{\longrightarrow} \cdots \stackrel{P_{m-1}}{\longrightarrow}\left(\hat{M}_{m}, \hat{\gamma}_{m}\right)=\left(M^{\prime}, \gamma^{\prime}\right) .
$$

In the case of a Type 5 modification, the relevant decomposing surface is a product disc.

We just have to check that Conditions (i)-(v) of 6.4 hold if $S^{\prime}$ satisfies Conditions 1-5. Since $S^{\prime}$ is standard, (i) is trivially true. We claim that 
Conditions 1, 2 and 4 guarantee (iii). Conditions 1 and 4 ensure that there is only a finite number of possibilities for each curve of $S^{\prime} \cap \partial \mathcal{H}^{0}$, up to ambient isotopy which keeps $\gamma$ and each handle invariant.

Lemma 10.1. Let $C$ and $C^{\prime}$ be two disjoint simple closed curves of $S^{\prime} \cap \partial \mathcal{H}^{0}$ where $S^{\prime}$ satisfies Conditions 1,2 and 4 . If there is an isotopy of $\partial \mathcal{H}^{0}$ which leaves $\gamma$ and each handle of $\mathcal{F}$ invariant and which takes $C$ onto $C^{\prime}$, then $C$ and $C^{\prime}$ are parallel in a way which respects $\gamma$ and the handle structure on $\mathcal{F}$.

Proof. Let $\alpha$ be an arc of $C \cap \mathcal{F}^{0}, C \cap \mathcal{F}^{1}$ or $C \cap \mathcal{R}_{ \pm}$, and let $\alpha^{\prime}$ be the image of $\alpha$ after the isotopy taking $C$ to $C^{\prime}$. It suffices to show that no arc of $C$ or $C^{\prime}$ lies between $\alpha$ and $\alpha^{\prime}$. Suppose that there is such an arc. If $\alpha$ lies in $\mathcal{F}^{1}$, then this means that Condition 1 is violated. If $\alpha$ lies in $\mathcal{R}_{ \pm}$, then Condition 4 is violated. If $\alpha$ lies in $\mathcal{F}^{0}$, then by Condition $4, C$ must intersect $\mathcal{F}^{0}$ in two arcs which are joined by two arcs in $\mathcal{R}_{ \pm}$. If $V$ is the 0 -handle of $\mathcal{F}$ containing $\alpha \cup \alpha^{\prime}$, then some sub-arc of $\partial V$ is a tubing arc for $S^{\prime}$, contrary to Condition 2.

Hence, there is only a finite number of possible arrangements for $S^{\prime} \cap \partial \mathcal{H}^{0}$, up to possibly taking multiple parallel copies of each curve and performing an ambient isotopy which leaves $H_{0}, H_{0} \cap \mathcal{F}$ and $H_{0} \cap \gamma$ invariant. Consider therefore a collection $C$ of $n$ parallel curves of $S^{\prime} \cap \partial \mathcal{H}^{0}$, the parallelity regions respecting $\mathcal{F}$ and $\gamma$. Let $H_{1}^{\prime}, \ldots, H_{n-1}^{\prime}$ be the associated 0 -handles of $\mathcal{H}^{\prime}$ lying between them. There are three possibilities: Either each curve of $C$ lies entirely in $\mathcal{F}$, or each curve of $C$ is disjoint from $\mathcal{F}$, or each curve of $C$ hits $\partial \mathcal{F}$. In the first case, $\mathcal{F}^{\prime} \cap H_{i}^{\prime}$ is an annulus disjoint from $\gamma^{\prime}$ for each $i$, and so none of the $H_{i}^{\prime}$ lie in $\mathcal{I} H^{0}\left(M^{\prime}\right)$, and hence can be ignored. Similarly, in the second case, none of the $H_{i}^{\prime}$ lie in $\mathcal{I} H^{0}\left(M^{\prime}\right)$. In the final case, we claim that at most one $H_{i}^{\prime}$ lies in $\mathcal{I} H^{0}\left(M^{\prime}\right)$. For if two adjacent curves of $C$ are coherently oriented, then $\mathcal{F}^{\prime}$ intersects the 0-handle between them in a collection of product discs. Hence, this 0-handle does not lie in $\mathcal{I} H^{0}\left(M^{\prime}\right)$. If two adjacent curves of $C$ are incompatibly oriented (say they point towards each other), then the arcs of $\partial \mathcal{F}$ lying between them must all point out of $\mathcal{F}$. Otherwise, Condition 2 is violated. Hence, at most one pair of adjacent curves of $C$ can be incoherently oriented. In particular, at most one $H_{i}^{\prime}$ can lie in $\mathcal{I} H^{0}\left(M^{\prime}\right)$. Therefore, (iii) of 6.4 is established.

We will now focus on a component $F$ of $\mathcal{F}$, and will compare its complexity with the complexity of $F^{\prime}=F \cap \mathcal{F}^{\prime}$.

Lemma 10.2. Let $S^{\prime}$ be a standard surface satisfying Condition 1. Then no simple closed curve of $S^{\prime} \cap F$ bounds a disc in $F$.

Proof. We may pick such a simple closed curve to be innermost in $F$, bounding a disc $D$, which inherits a handle structure from $F$. Since $D$ is a disc, 
there is a 0 -handle of $D$ with valence at most one. If this 0 -handle has valence zero, then $S^{\prime}$ is not standard. If this 0 -handle has valence one, then Condition 1 is violated.

The following corollary of Lemma 10.2 is a simple property of planar surfaces:

Corollary 10.3. Let $S^{\prime}$ be a standard surface satisfying Condition 1 . Then one of the following holds:

- Each component $X$ of $F^{\prime}$ has $|\partial X|<|\partial F|$, or

- $F^{\prime}$ is obtained from $F$ by cutting along arcs and circles which are parallel to arcs and circles in $\partial F$.

Condition 1 also has the following implication:

Lemma 10.4. Let $S^{\prime}$ be a standard surface satisfying Condition 1. Then any component of $F^{\prime}$ meets any 1-handle of $F$ in at most one disc.

Proof. Suppose, on the contrary, that there is a component $X$ of $F^{\prime}$ meeting a 1-handle $D$ of $\mathcal{F}$ in more than one disc. Let $D_{1}$ and $D_{2}$ be two discs of $X \cap D$, and let $\alpha_{1}$ be an arc in $X$ joining $D_{1}$ to $D_{2}$. Let $\alpha_{2}$ be an $\operatorname{arc}$ in $D$ transverse to $S^{\prime} \cap D$, joining the endpoints of $\alpha_{1}$, in such a way that $\alpha_{1} \cup \alpha_{2}$ forms a simple closed curve, which bounds a disc $D^{\prime}$ in $\partial \mathcal{H}^{0}(M)$. There exists at least one circle $C$ of $S^{\prime} \cap \partial \mathcal{H}^{0}$ entering $D^{\prime}$ through $\alpha_{2}$. This arc cannot leave $D^{\prime}$ through $\alpha_{1}$ and so must leave $D^{\prime}$ through $\alpha_{2}$. Hence, $C$ violates Condition 1.

Lemma 10.5. Let $S^{\prime}$ be a standard surface satisfying Conditions 1,2 and 3. Let $D$ be a component of $F^{\prime}$ with a negative index 0-handle. Then there is a 1-handle of $F$ which lies entirely in $D$.

Proof. The 0-handle $V$ of $D$ must be disjoint from $\gamma^{\prime}$ and have valence at most one. The boundary of $V$ is divided into $\partial V \cap \partial F, \partial V \cap S^{\prime}$ and at most one arc $V \cap \mathcal{F}^{1}\left(M^{\prime}\right)$. If $\alpha$ is an arc of $\partial V \cap \partial F$ with both endpoints lying in $S^{\prime}$, then $\alpha$ is a tubing arc, contrary to Condition 2 .

Suppose first that $V$ has zero valence. Then, $\partial V$ is divided into $\partial V \cap \partial F$ and $\partial V \cap S^{\prime}$. However, we cannot have an arc of $\partial V \cap \partial F$, since its endpoints would lie in $S^{\prime}$ and so would be a tubing arc. Hence, $\partial V$ lies wholly in $S^{\prime}$. But this violates the assumption that $S^{\prime}$ is standard, which is a contradiction.

Now suppose that $V$ has valence one, with a single 1-handle $E$ of $\mathcal{F}^{\prime}$ attached to it. Let $\beta_{1}$ and $\beta_{2}$ be the two arcs of $\partial E \cap \partial D$. Then each $\beta_{i}$ originally came from $\partial F$ or from $S^{\prime} \cap F$.

If both $\beta_{1}$ and $\beta_{2}$ lie inside $S^{\prime} \cap F$, then the arc $\partial V-\partial E$ also lies inside $S^{\prime} \cap F$, for otherwise there would be an arc of $\partial V \cap \partial F$ with endpoints in $S^{\prime}$, violating Condition 2. But if $\partial V-\partial E$ lies wholly in $S^{\prime}$, then Condition 1 is violated. Similarly, if $\beta_{1}$ lies inside $S^{\prime} \cap F$ and $\beta_{2}$ lies inside $\partial F$, then 
Condition 3 is violated. If both $\beta_{1}$ and $\beta_{2}$ lie inside $\partial F$, then $E$ is the required 1-handle of $F$ lying solely in $D$.

An example of a component $D$ of $F^{\prime}$ with $I(D)<0$ is given in Fig. 5.2.

Proposition 10.6. Let $F$ be a component of $\mathcal{F}$, and let $F^{\prime}=F \cap \mathcal{F}^{\prime}$. Suppose that every 0-handle of $F$ has positive index. If $S$ is a standard surface satisfying Conditions 1-5, then $C_{\mathcal{F}}\left(F^{\prime}\right) \leq C_{\mathcal{F}}(F)$. Also, if we have equality, then each component of $S^{\prime} \cap F$ is a circle parallel to a component of $\partial F$ disjoint from $\gamma$. The parallelity region inherits a handle structure from $\mathcal{F}$ in which each 0 -handle has valence two.

Proof. Suppose that $C_{\mathcal{F}}\left(F^{\prime}\right) \geq C_{\mathcal{F}}(F)$. By Lemma 10.4, each component $Y$ of $F^{\prime}$ has $C_{1}(Y) \leq C_{1}(F)$. If some component $D$ of $F^{\prime}$ has negative index, then some 0 -handle of $D$ has negative index and so by Lemma 10.5, all remaining components $Y$ of $F^{\prime}$ have $C_{1}(Y)<C_{1}(F)$. But by definition $D$ does not contribute to the $\mathcal{F}$-complexity of $F^{\prime}$. Hence $C_{\mathcal{F}}\left(F^{\prime}\right)<C_{\mathcal{F}}(F)$, which is contrary to assumption. Thus no component of $F^{\prime}$ has negative index. Hence, the index of $F$ is shared among the components of $F^{\prime}$. Since $C_{\mathcal{F}}\left(F^{\prime}\right) \geq C_{\mathcal{F}}(F)$, then one component $X$ of $F^{\prime}$ has $C_{1}(X)=C_{1}(F)$ and $I(X)=I(F)$. All other components $Y$ of $F^{\prime}$ have zero index, and so, by definition, they do not contribute to the $\mathcal{F}$-complexity of $F^{\prime}$. By Corollary 10.3, $|\partial X| \leq|\partial F|$. Hence, $C_{\mathcal{F}}\left(F^{\prime}\right) \leq C_{\mathcal{F}}(F)$, and so $C_{\mathcal{F}}\left(F^{\prime}\right)=C_{\mathcal{F}}(F)$.

We now wish to examine further the case when $C_{\mathcal{F}}\left(F^{\prime}\right)=C_{\mathcal{F}}(F)$. Since $|\partial X|=|\partial F|$, Corollary 10.3 implies that $F^{\prime}$ is obtained from $F$ by cutting along arcs and circles which are parallel to arcs and circles in $\partial F$. Each component of $F^{\prime}-X$ has index zero. If $V$ is a 0 -handle of $F^{\prime}$ not lying in $X$, then $V$ cannot have negative index. For otherwise, Lemma 10.5 would imply that $C_{1}(X)<C_{1}(F)$. Thus, each 0-handle $V$ of $F^{\prime}-X$ must have zero index.

We will now show that in fact there are no index zero discs of $F^{\prime}$. If there is such a disc, then there is an arc of $F \cap S^{\prime}$ extrememost in $F$, parallel to an arc in $\partial F$ via a parallelity disc $D$. If this disc $D$ does not have zero index, then $D=X$ and hence $F$ is a disc. In this case, we may find an arc of $F \cap S^{\prime}$ which is extrememost in $F$ and which does separate off an index zero disc. Thus, we may assume that $D$ has zero index. Let $V$ be a 0 -handle of $D$ with valence at most one. Since $V$ has zero index, there are two cases to consider. If $V$ has valence zero and hits $\gamma^{\prime}$ twice, then Condition 5 is violated. If $V$ has valence one and hits $\gamma^{\prime}$ once, then Condition 3 is violated.

Hence, each component of $F \cap S^{\prime}$ is a simple closed curve parallel to a curve of $\partial F$. An extrememost component of $F \cap S^{\prime}$ separates off an annulus $A$. If $A=X$, then $F$ is an annulus, in which case we may find an arc of $F \cap S^{\prime}$ extrememost in $F$ which is parallel to a component of $\partial F$ via a component of $F^{\prime}$ other than $X$. Hence, we may assume $A \neq X$. Therefore, $A$ has zero index, and so each 0 -handle of $A$ has valence two and is disjoint 
from $\gamma$. Repeating this argument for each component of $F \cap S^{\prime}$ proves the proposition.

The following verifies (ii), (iv) and (v) of Proposition 6.4 and completes the proof of that proposition and hence of Theorems 1.4, 1.5 and 1.6:

Proposition 10.7. Suppose that every 0-handle of $\mathcal{F}$ has positive index. Suppose also that $H_{0} \cap(\mathcal{F}(M) \cup \gamma)$ is connected for each 0-handle $H_{0}$ of $\mathcal{H}(M)$. Let $S^{\prime}$ be a standard surface satisfying Conditions 1-5, with $\left[S^{\prime}, \partial S^{\prime}\right]$ $\neq 0 \in H_{2}(M, \partial M)$. Then $C\left(H_{0} \cap \mathcal{H}^{\prime}\right) \leq C\left(H_{0}\right)$ for each 0 -handle $H_{0}$ of $\mathcal{H}$. Also, this inequality is strict for some 0-handle $H_{0}$. If this inequality is an equality for some 0 -handle $H_{0}$, then $H_{0} \cap \mathcal{H}^{\prime}$ is obtained from $H_{0}$ by a trivial modification.

Proof. Suppose that $C\left(H_{0} \cap \mathcal{H}^{\prime}\right) \geq C\left(H_{0}\right)$ for some 0-handle $H_{0}$ of $\mathcal{H}$. Then $C_{\mathcal{F}}\left(H_{0} \cap \mathcal{H}^{\prime}\right) \geq C_{\mathcal{F}}\left(H_{0}\right)$. But, by Proposition 10.6, each component $F$ of $\mathcal{F}$ has $C_{\mathcal{F}}\left(F \cap \mathcal{F}^{\prime}\right) \leq C_{\mathcal{F}}(F)$. Hence $C_{\mathcal{F}}\left(H_{0} \cap \mathcal{H}^{\prime}\right) \leq C_{\mathcal{F}}\left(H_{0}\right)$. Therefore, for each component $F$ of $\mathcal{F} \cap H_{0}$, we must have $C_{\mathcal{F}}\left(F \cap \mathcal{F}^{\prime}\right)=C_{\mathcal{F}}(F)$. Proposition 10.6 then implies that each component of $S^{\prime} \cap \mathcal{F} \cap H_{0}$ is a circle parallel to a component of $\partial \mathcal{F}$ disjoint from $\gamma$. Therefore, $n\left(\mathcal{H}^{\prime} \cap H_{0}\right) \geq$ $n\left(H_{0}\right)=1$. Hence, $C\left(\mathcal{H}^{\prime} \cap H_{0}\right) \leq C\left(H_{0}\right)$.

Suppose that this is an equality for some 0 -handle $H_{0}$ of $\mathcal{H}$. Then, as above, this implies that $n\left(\mathcal{H}^{\prime} \cap H_{0}\right)=n\left(H_{0}\right)=1$. Also, the argument above gives that each component of $S^{\prime} \cap \mathcal{F} \cap H_{0}$ is a circle parallel to a component of $\partial \mathcal{F}$ disjoint from $\gamma$. This component of $\partial \mathcal{F}$ bounds a disc in $\partial H_{0}$ with interior disjoint from $\mathcal{F}$, since $H_{0} \cap(\mathcal{F}(M) \cup \gamma)$ is connected. Hence, $H_{0} \cap \mathcal{H}^{\prime}$ is obtained from $H_{0}$ by a trivial modification.

Suppose now that $C\left(H_{0} \cap \mathcal{H}^{\prime}\right)=C\left(H_{0}\right)$ for every 0-handle $H_{0}$ of $\mathcal{H}$. We aim to achieve a contradiction. Let $C$ be the collection of circles of $S^{\prime} \cap \mathcal{F}$ extrememost in $\mathcal{F}$. Then, there is a collection of annuli $A$ in $\mathcal{F}$ which is disjoint from $\gamma$ and with $A \cap S^{\prime}=C$. Let $D$ be the collection of discs of $S^{\prime} \cap \mathcal{H}^{0}$ which $C$ bounds. Then $A \cup D$ is a collection of discs properly embedded in $M$ which are parallel to discs in $\mathcal{R}_{ \pm}$via balls $B_{0}$. These balls lie in $\mathcal{H}^{0}$ since $H_{0} \cap(\mathcal{F}(M) \cap \gamma)$ is connected for each 0-handle $H_{0}$ of $\mathcal{H}(M)$.

For each 1-handle $H_{1}=D^{2} \times[0,1]$, the discs $D^{2} \times\{0\}$ and $D^{2} \times\{1\}$ are each divided up by the decomposition along $S^{\prime}$. For $i=0$ and 1 , all but one 0 -handle of $D^{2} \times\{i\}-\operatorname{int}\left(\mathcal{N}\left(S^{\prime}\right)\right)$ has index zero. The remaining component has index equal to the index of $D^{2} \times\{i\}$. But the index of $D^{2} \times\{i\}$ is positive, since we are assuming that the index of each 0 -handle of $\mathcal{F}$ is positive. Hence, the product structure on $H_{1}$ matches $A \cap\left(D^{2} \times\{0\}\right)$ with $A \cap\left(D^{2} \times\{1\}\right)$. We may therefore unambiguously define $A \cap D^{2}$. Let $B_{1}$ be the union (over all 1-handles) of the balls $\left(A \cap D^{2}\right) \times[0,1]$. Similarly, we may find a collection $B_{2}$ of components of $\mathcal{H}^{2}-\operatorname{int}\left(\mathcal{N}\left(S^{\prime}\right)\right)$, and such that $B_{2} \cap \mathcal{H}^{0}=\mathcal{H}^{2} \cap A$. 
Then $B_{0} \cup B_{1} \cup B_{2}$ is a parallelity region between some closed components of $S^{\prime}$ and a subsurface of $\mathcal{R}_{ \pm}$. If we remove these components, we may repeat the argument, and show eventually that each component of $S^{\prime}$ which touches $\mathcal{F}$ is closed and parallel to some component of $\mathcal{R}_{ \pm}$. This does not quite show that $\left[S^{\prime}, \partial S^{\prime}\right]=0 \in H_{2}(M, \partial M)$, since there may be components of $S^{\prime}$ which are disjoint from $\mathcal{F}$. Such a component $X$ lies entirely in a 0 -handle $H_{0}$ of $\mathcal{H}$. But recall from above that $n\left(\mathcal{H}^{\prime} \cap H_{0}\right)=n\left(H_{0}\right)=1$. Hence, $\partial X$ cannot separate components of $H_{0} \cap \mathcal{F}$. In particular, $X$ is parallel to a disc in $\partial M$. Therefore, $\left[S^{\prime}, \partial S^{\prime}\right]=0 \in H_{2}(M, \partial M)$, contrary to assumption.

\section{The algorithm to construct the tangles.}

In this section, we demonstrate how to construct the graphs $G$ required for algorithm of Theorem 1.4 which we outlined in Section 2. Recall that each graph $G$ is embedded in a 3 -simplex $\Delta^{3}$ and comes with a regular neighbourhood $\mathcal{N}(G)$ and arcs labelled $\gamma$ and $\tau$ in $\partial \mathcal{N}(G)$. Recall that the $\operatorname{arcs} \gamma$ form the tangles required for Theorems 1.5 and 1.6.

In line with the rest of this paper, we work with the handle structure $\mathcal{H}$ arising by dualising the given generalised triangulation of $M$. We will focus on a single 0 -handle $H_{0}$ of $\mathcal{H}$. The algorithm starts with the 0 -handle $H_{0}$ and the surface $\mathcal{F}(M) \cap H_{0}$. This surface is one of finitely many possibilities, but, for the moment, we will assume that $\mathcal{F}(M) \cap H_{0}$ is as in Figure 11.1. In general, it may be a subsurface of this; we will explain later how to cope with this eventuality.

At each stage $j \in \mathbb{N}$ of the algorithm, we will construct a finite list of possibilities for the following objects lying in $H_{0}$ :

- A subset $\mathcal{H}_{j}^{0}$ of $H_{0}$, which is a union of 3-balls embedded in $H_{0}$,

- a subsurface $\mathcal{F}\left(\mathcal{H}_{j}\right)$ of $\mathcal{H}_{j}^{0} \cap \mathcal{F}(M)$, and

- $\operatorname{arcs} \gamma_{j}$ properly embedded in $\operatorname{cl}\left(\partial \mathcal{H}_{j}^{0}-\mathcal{F}\left(\mathcal{H}_{j}\right)\right)$.

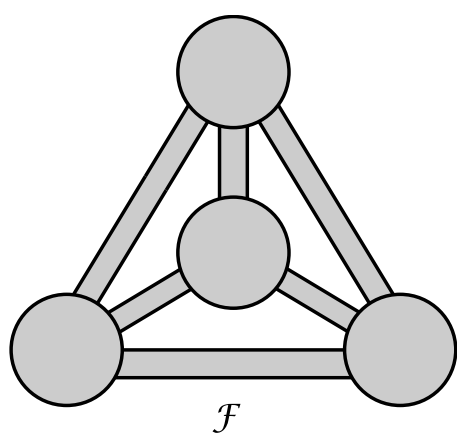

Figure 11.1. 
Each component of $\partial \mathcal{H}_{j}^{0}-\left(\mathcal{F}\left(\mathcal{H}_{j}\right) \cup \gamma_{j}\right)$ will have a specified orientation, pointing into or out of $\mathcal{H}_{j}^{0}$. When we wish to refer to the above data, we will denote it simply by $\mathcal{H}_{j}$.

For $j=1$, we take $\mathcal{H}_{1}^{0}=H_{0}, \mathcal{F}\left(\mathcal{H}_{1}\right)=H_{0} \cap \mathcal{F}(M)$, and $\gamma_{1}=\emptyset$. We consider all possible orientations for $\partial \mathcal{H}_{1}^{0}-\mathcal{F}\left(\mathcal{H}_{1}\right)$. If (as we supposed above) $H_{0} \cap \mathcal{F}(M)$ is as in Figure 11.1, then there are four components of $\partial \mathcal{H}_{1}^{0}-\mathcal{F}\left(\mathcal{H}_{1}\right)$, and so there are 16 possible orientations.

The algorithm constructs the list of possibilities for $\mathcal{H}_{j+1}$ by considering each possibility for $\mathcal{H}_{j}$ in turn, and performing some modifications to it, which we describe below. These modifications have the property that, if $\mathcal{H}_{j}$ is some fixed possibility at the $j^{\text {th }}$ stage, then each possibility for $\mathcal{H}_{j+1}$ to which it gives rise satisfies one of the following:

- $C\left(\mathcal{H}_{j+1}\right)<C\left(\mathcal{H}_{j}\right)$ or

- $C\left(\mathcal{H}_{j+1}\right)=C\left(\mathcal{H}_{j}\right)$ and $C^{+}\left(\mathcal{H}_{j+1}\right)<C^{+}\left(\mathcal{H}_{j}\right)$.

Thus, by Lemma 5.3, the algorithm will terminate at stage $m$, say. However, we do not know this value of $m$ until we run the algorithm.

It should be clear that this algorithm is modelling within the single 0handle $H_{0}$ what is happening in the Proof of Theorem 1.4. Recall that, in that proof, we constructed a sequence of sutured manifolds embedded within $M$, and examined how each sutured manifold $\left(M_{i}, \gamma_{i}\right)$ intersected any given 0 -handle $H_{0}$. However, the intersection $M_{i} \cap H_{0}$ does not necessarily correspond precisely with the $i^{\text {th }}$ stage of the algorithm we are about to outline. This is because, when passing from a single possibility for $\mathcal{H}_{j}$ to several possibilities for $\mathcal{H}_{j+1}$, we insist that complexity or extended complexity strictly decreases within our given 0 -handle. However, at each stage in the induction of Theorem 1.4, we merely insisted that complexity decreased within some 0 -handle (not necessarily the one we are examining). Therefore, in order to determine how the final sutured manifold $M_{n}$ lies in $H_{0}$, we must consider every possibility for $\mathcal{H}_{j}$, where $1 \leq j \leq m$. Given one such possibility $\mathcal{H}_{j}$, we construct the graph $G$ by associating a vertex of $G$ with each component of $\mathcal{H}_{j}^{0}$; we associate an edge of $G$ with each component of $\mathcal{F}^{0}\left(\mathcal{H}_{j}\right)$; the curves $\tau$ are specified by $\mathcal{F}^{1}\left(\mathcal{H}_{j}\right)$; the arcs $\gamma$ are formed by taking all possible subtangles of $\gamma_{j}$.

We now give the heart of the algorithm, namely the procedure which constructs each possibility for $\mathcal{H}_{j+1}$ arising from a single possibility for $\mathcal{H}_{j}$. We apply one of the following procedures to $\mathcal{H}_{j}$ (and we give the points in Sections 7-9 where we applied them):

\section{Removal of a component of $\mathcal{H}_{j}^{0}$.}

This can occur in Procedures 1 and 5 of Section 7. It can also occur in Cases 1, 2, 3 and 4B of Section 8. When a component of $\mathcal{H}_{j}^{0}$ is removed, so are the components of $\mathcal{F}\left(\mathcal{H}_{j}\right)$ and arcs of $\gamma_{j}$ which it contains. 
2. Removing some handles of $\mathcal{F}\left(\mathcal{H}_{j}\right)$ disjoint from $\gamma_{j}$.

In order that the new surface inherits a handle structure, we insist that if a 0 -handle of $\mathcal{F}\left(\mathcal{H}_{j}\right)$ is removed, then so are the 1 -handles of $\mathcal{F}\left(\mathcal{H}_{j}\right)$ which abut it. Also, we may only perform this procedure if the components of $\partial \mathcal{H}_{j}^{0}-\left(\mathcal{F}\left(\mathcal{H}_{j}\right) \cup \gamma_{j}\right)$ which touch any removed handle have orientations which agree. This operation can occur in Procedures 1, 2, 5 and 6 of Section 7, and Case 4B of Section 8.

Note that, in general, $\mathcal{F}(M) \cap H_{0}$ is obtained from the surface in Figure 11.1 by removing some handles. Therefore, by applying this procedure at the first stage $j=1$, we can incorporate all possibilities for $\mathcal{F}(M) \cap H_{0}$ into this algorithm.

3. Replacing handles of $\mathcal{F}\left(\mathcal{H}_{j}\right)$ with a sub-arc of $\gamma_{j+1}$.

Here, we may replace a 1 -handle of $\mathcal{F}\left(\mathcal{H}_{j}\right)$ with an arc of $\gamma_{j+1}$, providing that the components of $\partial \mathcal{H}_{j}^{0}-\left(\mathcal{F}\left(\mathcal{H}_{j}\right) \cup \gamma_{j}\right)$ which touch this handle have orientations which disagree. We may also remove a 0 -handle of $\mathcal{F}\left(\mathcal{H}_{j}\right)$ which has valence one and which intersects $\gamma_{j}$ in a single point, providing that we also remove the 1-handle of $\mathcal{F}\left(\mathcal{H}_{j}\right)$ which it abuts and we then replace these handles with a sub-arc of $\gamma_{j+1}$. This occurs in Procedure 3 of Section 7 and in Case 3 of Section 8.

4. Removal of a product disc component of $\mathcal{F}\left(\mathcal{H}_{j}\right)$.

If $F$ is a disc component of $\mathcal{F}\left(\mathcal{H}_{j}\right)$ intersecting $\gamma_{j}$ twice, we may replace $F$ with an arc of $\gamma_{j+1}$ joining the two points of $F \cap \gamma_{j}$. This occurs in Procedure 4 of Section 7.

5. Removal of a valence two 0-handle of $\mathcal{F}\left(\mathcal{H}_{j}\right)$.

If $V$ is a 0 -handle of $\mathcal{F}\left(\mathcal{H}_{j}\right)$ which is disjoint from $\gamma_{j}$ and which abuts two distinct 1-handles of $\mathcal{F}\left(\mathcal{H}_{j}\right)$, then we may combine $V$ and the two 1-handles into a single 1-handle of $\mathcal{F}\left(\mathcal{H}_{j+1}\right)$. This occurs in Case 1 of Section 8.

6. Decomposition along a surface.

This step models the sutured manifold decomposition outlined in Section 9. We only perform this operation providing each 0-handle of $\mathcal{F}\left(\mathcal{H}_{j}\right)$ has positive index and providing $H_{0} \cap\left(\mathcal{F}\left(\mathcal{H}_{j}\right) \cup \gamma_{j}\right)$ is connected for each 0 -handle $H_{0}$ of $\mathcal{H}_{j}$. We construct all possible oriented curves $C$ which satisfy Conditions 1-5 of Section 9 (viewing $C$ as a possibility for $S^{\prime} \cap \partial \mathcal{H}^{0}$ ). There is only a finite number of possibilities $\left(C_{1}, \ldots, C_{t}\right.$, say) for $C$. We then let $C^{\prime}$ be a collection of disjoint simple closed curves in $\partial \mathcal{H}_{j}^{0}$, each curve being a copy of one of the $C_{i}$ 's, and with no two components of $C^{\prime}$ representing the same $C_{i}$ (although, two components of $C^{\prime}$ may be the same underlying curve, but have opposite orientations). We insist that $C^{\prime}$ also satisfies Condition 2 of Section 9 . We then extend $C^{\prime}$ to a collection of disjoint discs properly embedded in $\mathcal{H}_{j}^{0}$. We then decompose $\mathcal{H}_{j}^{0}$ along these discs, creating a new 
collection of 0-handles $\mathcal{H}_{j+1}^{0}$, which naturally inherit $\mathcal{F}\left(\mathcal{H}_{j+1}\right)$ and sutures $\gamma_{j+1}$. By the argument of Propositions 10.6 and 10.7, $C\left(\mathcal{H}_{j+1}\right)<C\left(\mathcal{H}_{j}\right)$, unless each component of $C^{\prime}$ is a curve lying entirely in $\mathcal{F}\left(\mathcal{H}_{j}\right)$ parallel to some component of $\partial \mathcal{F}\left(\mathcal{H}_{j}\right)$ disjoint from $\gamma_{j}$, the parallelity region respecting the handle structure of $\mathcal{F}\left(\mathcal{H}_{j}\right)$. In this case, the modification is trivial. We therefore do not include this case as a possibility for $\mathcal{H}_{j+1}$. However, the modification may alter the orientations of some components of $\partial \mathcal{H}_{j}^{0}-\mathcal{F}\left(\mathcal{H}_{j}\right)$ disjoint from $\gamma_{j}$. We therefore have to consider all possible orientations for these components as giving distinct possibilities for $\mathcal{H}_{j}$.

\section{Amalgam removal.}

We only perform this operation when each component of $\mathcal{F}\left(\mathcal{H}_{j}\right)$ has positive index and each 0 -handle of $\mathcal{F}\left(\mathcal{H}_{j}\right)$ with nonpositive index has valence two and is disjoint from $\gamma_{j}$. Suppose that $D$ is a union of handles of $\mathcal{F}\left(\mathcal{H}_{j}\right)$ which forms a disc disjoint from $\gamma$. Suppose also that if $D$ has any 0-handles, then each such 0 -handle abuts precisely two 1-handles of $\mathcal{F}\left(\mathcal{H}_{j}\right)$, both of which lie in $D$. Suppose also that the two components of $\partial \mathcal{H}_{j}^{0}-\left(\mathcal{F}\left(\mathcal{H}_{j}\right) \cup \gamma_{j}\right)$ which touch $D$ have the same orientation. We take one or two copies of $\partial D$ and move them a little, creating a curve $C_{1}$ (and possibly $C_{2}$ ) which intersect $\mathcal{F}\left(\mathcal{H}_{j}\right)$ in a collection of arcs lying in 0-handles of $\mathcal{F}\left(\mathcal{H}_{j}\right)$. Extend each $C_{i}$ to a disc $D_{i}$ properly embedded in $\mathcal{H}_{j}^{0}$. If we have both $D_{1}$ and $D_{2}$, we orient them inconsistently, in a way which gives the parallelity region between them some sutures. If we are just dealing with $D_{1}$, we consider both possible orientations. We then decompose $\mathcal{H}_{j}^{0}$ along $D_{1}$ (and possibly $D_{2}$ ), providing this is not a trivial modification, as outlined in Operation 6 above. This occurs in Case 5 of Section 8 . Note that we cannot necessarily include this case here in Operation 6 , since the curves $C_{1}$ and $C_{2}$ might fail Conditions 2, 4 or 5 of Section 9.

The Figure 11.2 gives a concrete example of some of the above operations. It is clear that these procedures may implemented algorithmically, although they may pose some challenges for a computer programmer.

\section{Exceptional and norm-exceptional surgeries with $\Delta(\sigma, \mu)=1$.}

We now give examples which demonstrate that the restriction on $\Delta(\sigma, \mu)$ in Theorems 1.4, 1.5 and 1.6 is necessary. We give a method of constructing in a 3-manifold $M$ (satisfying certain conditions) an infinite number of surgery curves $K$ with exceptional or norm-exceptional surgery slopes $\sigma$ satisfying $\Delta(\sigma, \mu)=1$, where $\mu$ is the meridian slope on $\partial \mathcal{N}(K)$.

Let $M$ be a compact orientable 3-manifold with $\partial M$ a (possibly empty) union of tori. Suppose also that $M$ is irreducible, atoroidal and has incompressible boundary. Let $S$ be a connected oriented surface properly 


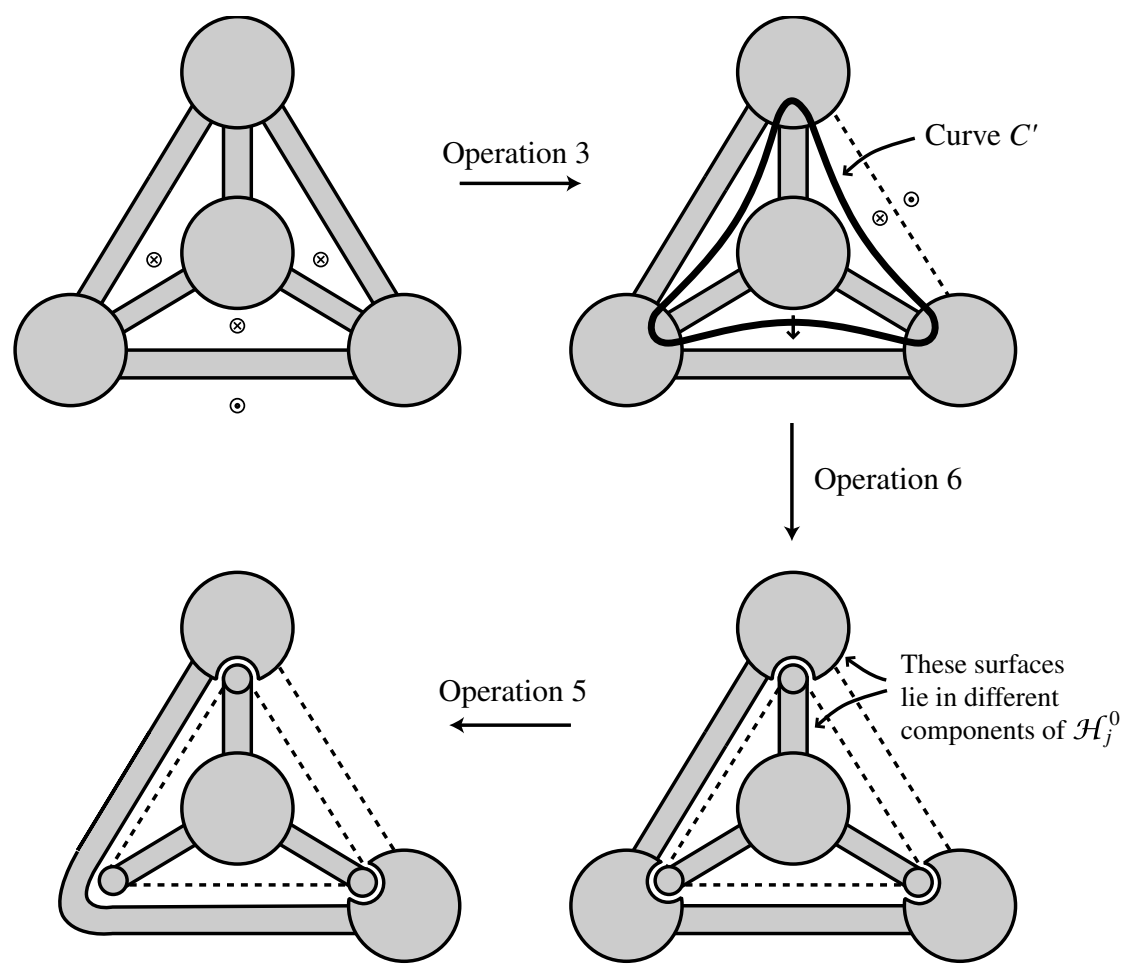

Key: $\odot$ Orientation out of $\mathcal{H}_{j}^{0}$

$\otimes$ Orientation into $\mathcal{H}_{j}^{0}$

Figure 11.2.

embedded in $M$ with $[S, \partial S] \neq 0 \in H_{2}(M, \partial M)$, and so that $S$ is incompressible and norm-minimising in its homology class. Then $S$ is neither a sphere nor a disc. Let $K$ be any essential simple closed curve on $S$ disjoint from $\partial S$. Let $\sigma$ be the slope of the curves $\partial \mathcal{N}(K) \cap S$, which is known as 'surface framing'.

Proposition 12.1. The slope $\sigma$ is exceptional or norm-exceptional.

Proof. The surface $S$ determines a class $z \in H_{2}(M-\operatorname{int}(\mathcal{N}(K)), \partial M)$ as follows: The two curves $S \cap \partial \mathcal{N}(K)$ divide $\partial \mathcal{N}(K)$ into two annuli. Attach either of these annuli to $S-\operatorname{int}(\mathcal{N}(K))$ and let $S^{\prime}$ be the resulting surface. Then $z=\left[S^{\prime}, \partial S^{\prime}\right] \in H_{2}(M-\operatorname{int}(\mathcal{N}(K)), \partial M)$ is independent of the choice of annulus. In fact, $S^{\prime}$ is norm-minimising in its class in $H_{2}(M-\operatorname{int}(\mathcal{N}(K)), \partial M)$, since $\chi_{-}\left(S^{\prime}\right)=\chi_{-}(S)=x([S, \partial S]) \leq x\left(\left[S^{\prime}, \partial S^{\prime}\right]\right)$. Let $z_{\sigma} \in H_{2}\left(M_{K}(\sigma), \partial M_{K}(\sigma)\right)$ be the image of $z$ under the map induced by inclusion. 
We may construct a surface $S_{\sigma}$ in $M_{K}(\sigma)$ by starting with $S-\operatorname{int}(\mathcal{N}(K))$ and attaching a disc to each curve of $S \cap \partial \mathcal{N}(K)$, the discs being meridian discs in the surgery solid torus. Then $\left[S_{\sigma}, \partial S_{\sigma}\right]=z_{\sigma} \in H_{2}\left(M_{K}(\sigma), \partial M_{K}(\sigma)\right)$. Also, $-\chi\left(S_{\sigma}\right)=-\chi\left(S^{\prime}\right)-2$. Since we assumed that $S$ was connected, there are two possibilities:

(i) $\chi_{-}\left(S_{\sigma}\right)<\chi_{-}\left(S^{\prime}\right)$, or

(ii) $\chi_{-}\left(S_{\sigma}\right)=\chi_{-}\left(S^{\prime}\right)=0$.

In Case (i), $x\left(z_{\sigma}\right)<x(z)$, and therefore $K$ and $\sigma$ are norm-exceptional. In Case (ii), $S_{\sigma}$ is a non-separating sphere or two non-separating discs in $M_{K}(\sigma)$. If $S_{\sigma}$ is a sphere, then $M_{K}(\sigma)$ is reducible. If $S_{\sigma}$ is two discs, then $M_{K}(\sigma)$ has compressible boundary, which implies that either $M_{K}(\sigma)$ is a solid torus or it is reducible. Thus, in this case, $K$ and $\sigma$ are exceptional.

We now show that one may find an infinite number of such knots $K$ on a given $S$ (satisfying some conditions), such that no two knots in this collection are ambient isotopic to each other in $M$.

Proposition 12.2. Let $M$ be a compact 3-manifold with $\partial M$ a (possibly empty) union of tori and with $H_{1}(M)$ torsion free. Let $S$ be a compact connected oriented surface properly embedded in $M$ which has positive genus and which is norm-minimising in its class in $\mathrm{H}_{2}(M, \partial M)$. Then, we may find an infinite collection of knots, each essential curves on $S$, no two of which are ambient isotopic in $M$.

Proof. We may find two simple closed curves $C_{1}$ and $C_{2}$ on $S$ which intersect each other precisely once. If one of these curves has infinite order in $H_{1}(M)$ $\left(C_{1}\right.$, say), then, for any integer $n$, consider the curve $n C_{1}+C_{2}$, which is constructed by taking $n$ (coherently oriented) parallel copies of $C_{1}$, together with $C_{2}$ and smoothing off the double-points. This is the required collection of knots on $S$.

Suppose therefore that $C_{1}$ and $C_{2}$ have finite order in $H_{1}(M)$. Since $H_{1}(M)$ is torsion free, this implies that $C_{1}$ and $C_{2}$ are homologically trivial. We will construct our collection of knots by analysing the 'Seifert form' on $S$. Given two disjoint homologically trivial closed curves $\alpha_{1}$ and $\alpha_{2}$ in $M$, define their linking number $l k\left(\alpha_{1}, \alpha_{2}\right)$ to be the signed intersection number between $\alpha_{2}$ and a (not necessarily embedded) Seifert surface for $\alpha_{1}$. This is independent of the choice of Seifert surface for $\alpha_{1}$, since any two Seifert surfaces can be glued to form a closed (not necessarily embedded) surface, with which $C_{2}$ has zero intersection, since it is homologically trivial. Also, it is symmetric: $l k\left(\alpha_{1}, \alpha_{2}\right)=l k\left(\alpha_{2}, \alpha_{1}\right)$. Given any curve $C$ on $S$, define $C^{+}$ to be the push-off of $C$ from $S$ in some specified normal direction. Define the framing $\operatorname{fr}(C)$ of any curve $C$ on $S$ which is homologically trivial in $M$ as $l k\left(C^{+}, C\right)$. Now,

$$
l k\left(C_{1}^{+}, C_{2}\right)-l k\left(C_{1}, C_{2}^{+}\right)= \pm 1,
$$


since $C_{1}$ and $C_{2}$ intersect in one point on $S$. This implies that

$$
l k\left(C_{1}^{+}, C_{2}\right)+l k\left(C_{1}, C_{2}^{+}\right) \neq 0 .
$$

Let $n_{1}$ be an arbitrary integer. Then

$$
\begin{aligned}
& f r\left(n_{1} C_{1}+C_{2}\right) \\
& =l k\left(\left(n_{1} C_{1}+C_{2}\right)^{+}, n_{1} C_{1}+C_{2}\right) \\
& =n_{1}^{2} l k\left(C_{1}^{+}, C_{1}\right)+n_{1}\left(l k\left(C_{2}^{+}, C_{1}\right)+l k\left(C_{1}^{+}, C_{2}\right)\right)+l k\left(C_{2}^{+}, C_{2}\right) \\
& =n_{1}^{2} k_{1}+n_{1} k_{2}+k_{3},
\end{aligned}
$$

for integers $k_{1}, k_{2}$ and $k_{3}$, where $k_{2}=l k\left(C_{1}, C_{2}^{+}\right)+l k\left(C_{1}^{+}, C_{2}\right) \neq 0$. Hence, $\operatorname{fr}\left(n_{1} C_{1}+C_{2}\right)$ takes infinitely many values.

We now claim that if $C$ and $C^{\prime}$ on $S$ are two closed curves on $S$ which are homologically trivial in $M$ and freely homotopic in $M$, then $\operatorname{fr}(C)=\operatorname{fr}\left(C^{\prime}\right)$. A free homotopy is realised by a map $f: A \rightarrow M$, where $A$ is an annulus and where $f(\partial A)=C \cup C^{\prime}$. We may ensure that $f$ respects the product structure on $\mathcal{N}(S)$ and hence that $f^{-1}(S)$ is $\partial A$, together with some properly embedded simple closed curves. We may also ensure that no region of $A-$ $\operatorname{int}\left(\mathcal{N}\left(f^{-1}(S)\right)\right)$ is a disc, and hence that $f^{-1}(S)$ is a collection $\alpha_{0}, \alpha_{1}, \ldots, \alpha_{n}$ of disjoint essential simple closed curves in $A$, where $\partial A=\alpha_{0} \cup \alpha_{n}$. Since the image of the annulus lying between $\alpha_{i}$ and $\alpha_{i+1}$ is disjoint from $S$, then $f r\left(\alpha_{i}\right)=f r\left(\alpha_{i+1}\right)$. Therefore, $f r(C)=f r\left(C^{\prime}\right)$.

Hence, we have constructed the required infinite collection of knots.

We now show that we may ensure that each knot $K$ in this infinite collection has $M-\operatorname{int}(\mathcal{N}(K))$ irreducible and atoroidal.

Proposition 12.3. Let $M$ and $S$ be as above. Then each essential simple closed curve $K$ on $S$ has $M-\operatorname{int}(\mathcal{N}(K))$ irreducible. Also, there are (up to ambient isotopy in $M)$ at most finitely many knots $K$ on $S$ for which $M-\operatorname{int}(\mathcal{N}(K))$ is toroidal.

Proof. Let $K$ be an essential simple closed curve on $S$. If $M-\operatorname{int}(\mathcal{N}(K))$ contains a reducing sphere, then this bounds a ball in $M$. By assumption, $M$ is irreducible, and so the knot $K$ must lie in this 3-ball, and is therefore homotopically trivial in $M$. However, $K$ is essential on $S$ and $S$ is $\pi_{1-}$ injective, since it is incompressible. This is a contradiction.

Suppose now that $M-\operatorname{int}(\mathcal{N}(K))$ is toroidal, and let $T$ be an essential torus in $M-\operatorname{int}(\mathcal{N}(K))$. Since $M$ is atoroidal, $T$ either is parallel in $M$ to a component of $\partial M$ or is compressible in $M$. Consider the former case, and let $T^{2} \times I$ be the parallelity region between $T$ and a component $T^{\prime}$ of $\partial M$. We may assume that the intersection $S \cap\left(T^{2} \times I\right)$ is a collection of discs and annuli, with $K$ being a core of one of these annuli. Hence, $K$ is parallel to a curve on $T^{\prime}$. It is not hard to show that if $K_{1}$ and $K_{2}$ are two curves on $S$ both parallel to curves in $T^{\prime}$, then either $K_{1}$ and $K_{2}$ are ambient isotopic in 
$M$ or $S$ contains a component parallel to $T^{\prime}$. However, $S$ is connected and nontrivial in $H_{2}(M, \partial M)$, which gives a contradiction.

Hence, we may restrict attention to the case where $T$ is compressible in $M$. This implies that $T$ bounds a solid torus or lies in a 3 -ball in $M$. We will show that the latter case cannot arise. For, $S-\operatorname{int}(\mathcal{N}(K))$ and $T$ are both essential in $M-\operatorname{int}(\mathcal{N}(K))$, and so we may isotope these surfaces in $M-\operatorname{int}(\mathcal{N}(K))$, so that no curve of $S \cap T$ bounds a disc in $T$ or $S-\operatorname{int}(\mathcal{N}(K))$. So no curve of $S \cap T$ bounds a disc in $S$. But if $T$ were to lie in a 3-ball in $M$, then each curve of $S \cap T$ would be homotopically trivial in $M$, and hence in $S$. Thus, $S \cap T$ would have to be empty. We now consider the intersection between $S$ and the compression disc for $T$. By an innermost curve argument, we may isotope $S$ and $K$, keeping them disjoint from $T$, so that afterwards they are disjoint from this disc. But then $T$ compresses in $M-\operatorname{int}(\mathcal{N}(K))$, which is a contradiction.

Thus, we may assume that $T$ bounds a solid torus $V$ in $M$. We may also assume that the surface $V \cap S$ is incompressible in $V$ and so is a collection of discs and annuli. The knot $K$ lies on one such annulus $A$. If $A$ has winding number one in $V$, then $K$ is a core of $V$ and so $T$ is parallel to $\partial \mathcal{N}(K)$, contradicting the assumption that $T$ is essential in $M-\operatorname{int}(\mathcal{N}(K))$. If $A$ has winding number greater than one in $V$, then a cabling annulus for $K$ is constructed by gluing $A-\operatorname{int}(\mathcal{N}(K))$ to the closure of one of the components of $T-A$. It is now not hard to show that $K$ is ambient isotopic to the core $K^{\prime}$ of an annular component $A^{\prime}$ of $V \cap S$, where $K^{\prime}$ has a cabling annulus disjoint from $S$. For the purposes of the proof of Proposition 12.3, we may consider the knot $K^{\prime}$ instead of $K$. Suppose therefore that the cabling annulus for $K$ is disjoint from $S$.

Claim. Let $K_{1}$ and $K_{2}$ be two essential simple closed curves on $S$, each cabled with cabling annulus disjoint from $S$. Suppose that the cabling annuli both lie on the same side of $S$. Then there is an isotopy of $S$ which takes $K_{1}$ off $K_{2}$.

We may take the cabling annulus $A_{i}$ for $K_{i}$ to be properly embedded in $M-\operatorname{int}(\mathcal{N}(S))$. Then a component of $M-\operatorname{int}\left(\mathcal{N}\left(S \cup A_{i}\right)\right)$ is a solid torus $V_{i}$. A simple examination of the intersection between the annulus $A_{1}$ and the solid torus $V_{2}$ establishes that we may isotope $A_{1}$ off $V_{2}$ unless the winding number of $A_{2}$ along is $V_{2}$ is two. Thus, the claim is proved unless the winding number of $A_{i}$ in $V_{i}$ is two, for both $i=1$ and 2 . In this case $V_{i}$ is an $I$-bundle over a Möbius band, with $V_{i} \cap \partial \mathcal{N}(S)$ being precisely the $\partial I$-bundle. Therefore, if $K_{1}$ and $K_{2}$ cannot be homotoped off each other, $V_{1} \cup V_{2}$ is an $I$-bundle over a connected non-orientable surface $G$ other than a Möbius band. The $I$-bundle over $\partial G$ is a collection of annuli. If any of these annuli are compressible, we may extend the $I$-bundle. Thus, we may construct an $I$-bundle $X$ over a compact connected non-orientable surface 
$G^{\prime}$, such that the $I$-bundle over $\partial G^{\prime}$ is incompressible in $M$, and so that $X \cap \partial \mathcal{N}(S)$ is the $\partial I$-bundle over $G^{\prime}$. If $G^{\prime}$ is a Möbius band, then there is an isotopy of $S$ taking $K_{1}$ off $K_{2}$. Suppose therefore that $G^{\prime}$ has negative Euler characteristic. Expand the $I$-bundle a little, so that the $\partial I$-bundle lies in $S$. If we remove the $\partial I$-bundle from $S$, and attach the $I$-bundle over $\partial G^{\prime}$, we create a surface $S^{\prime}$ with $\left[S^{\prime}, \partial S^{\prime}\right]=[S, \partial S] \in H_{2}(M, \partial M)$, and with $\chi\left(S^{\prime}\right)>\chi(S)$. This contradicts the assumption that $S$ is norm-minimising and incompressible.

There are at most finitely many disjoint essential non-parallel simple closed curves on $S$. This proves the proposition.

Propositions 12.1, 12.2 and 12.3 give the following result:

Theorem 12.4. Let $M$ be a compact orientable 3-manifold with $\partial M a$ (possibly empty) union of tori. Suppose that $M$ is irreducible and atoroidal, and has incompressible boundary. Suppose also that $H_{1}(M)$ is torsion free and that some nontrivial element of $H_{2}(M, \partial M)$ is represented by a normminimising incompressible surface with positive genus. Then (up to ambient isotopy) there is an infinite number of surgery curves $K$ in $M$, with exceptional or norm-exceptional surgery slopes $\sigma$ satisfying $\Delta(\sigma, \mu)=1$, where $\mu$ is the meridian slope on $\partial \mathcal{N}(K)$. We may ensure that each knot $K$ in this collection has $M-\operatorname{int}(\mathcal{N}(K))$ irreducible and atoroidal, and has $H_{2}(M-\operatorname{int}(\mathcal{N}(K)), \partial M) \neq 0$.

\section{References}

[1] D. Gabai, Foliations and the topology of 3-manifolds II, J. Differ. Geom., 26 (1987), 461-478, MR 89a:57014a, Zbl 0627.57012.

[2] W. Haken, Some results on surfaces in 3-manifolds, Studies in Modern Topology (1968), 39-98 MR 36 \#7118, Zbl 0194.24902.

[3] J. Hempel, 3-Manifolds, Ann. of Math. Studies, 86, Princeton Univ. Press, Princeton, N.J., 1976, MR 54 \#3702, Zbl 0345.57001.

[4] W. Jaco, Lectures on Three-Manifold Topology, Regional Conference Series in Mathematics, 43, Providence, 1980, A.M.S., MR 81k:57009, Zbl 0433.57001.

[5] W. Jaco and U. Oertel, An algorithm to decide if a 3-manifold is Haken, Topology, 23 (1984), 195-209, MR 85j:57014, Zbl 0545.57003.

[6] M. Lackenby, Surfaces, surgery and unknotting operations, Math. Ann., 308 (1997), 615-632, MR 98j:57028, Zbl 0876.57015.

[7] _ Dehn surgery on knots in 3-manifolds, J. Amer. Math. Soc., 10 (1997), 835864, MR 98h:57034, Zbl 0889.57014.

[8] _ Upper bounds in the theory of unknotting operations, Topology, 37 (1998), 63-73, MR 99f:57009, Zbl 0913.57006.

[9] Y. Mathieu, Unknotting, knotting by twists and property $(P)$ for knots in $S^{3}$, Knots 90 (ed. A. Kawauchi), Walter de Gruyter \& Co (1992), 93-102, MR 93i:57008, Zbl 0772.57012 . 
[10] M. Scharlemann, Sutured manifolds and generalized Thurston norms, J. Differ. Geom., 29 (1989), 557-614, MR 90e:57021, Zbl 0673.57015

[11] A. Thompson, Thin position and the recognition problem for $S^{3}$, Math. Res. Lett., 1 (1994), 613-630, MR 95k:57015, Zbl 0849.57009.

[12] J. Tollefson and N. Wang, Taut normal surfaces, Topology, 35 (1996), 55-75, MR 97j:57022, Zbl 0868.57022.

[13] F. Waldhausen, The word problem in fundamental groups of sufficiently large irreducible 3-manifolds, Ann. Math., 88 (1968), 272-280, MR 39 \#2167, Zbl 0167.52103.

Received July 20, 1999.

\author{
Mathematical Institute \\ OXFORD UNIVERSITY \\ 24-29 St GiLES' \\ OXFORD OX1 3LB \\ UNITED KINGDOM \\ E-mail address: lackenby@maths.ox.ac.uk
}

